\title{
Spin-Atomic Vibration Interaction and Spin-Flip Hamiltonian of a Single Atomic Spin in a Crystal Field
}

\author{
Satoshi Kokado*, Kikuo Harigaya ${ }^{1}$, and Akimasa Sakuma ${ }^{2}$ \\ Faculty of Engineering, Shizuoka University, Hamamatsu 432-8561, Japan \\ ${ }^{1}$ Nanosystem Research Institute, AIST, Tsukuba 305-8568, Japan \\ ${ }^{2}$ Graduate School of Engineering, Tohoku University, Sendai 980-8579, Japan
}

\begin{abstract}
We derive the spin-atomic vibration interaction $V_{\mathrm{SA}}$ and the spin-flip Hamiltonian $V_{\mathrm{SF}}$ of a single atomic spin in a crystal field. We here apply the perturbation theory to a model with the spin-orbit interaction and the kinetic and potential energies of electrons. The model also takes into account the difference in vibration displacement between an effective nucleus and electrons, $\Delta \boldsymbol{r}$. Examining the coefficients of $V_{\mathrm{SA}}$ and $V_{\mathrm{SF}}$, we first show that $V_{\mathrm{SA}}$ appears for $\Delta \boldsymbol{r} \neq 0$, while $V_{\mathrm{SF}}$ is present independently of $\Delta \boldsymbol{r}$. As an application, we next obtain $V_{\mathrm{SA}}$ and $V_{\mathrm{SF}}$ of an Fe ion in a crystal field of tetragonal symmetry. It is found that the magnitudes of the coefficients of $V_{\mathrm{SA}}$ can be larger than those of the conventional spin-phonon interaction depending on vibration frequency. In addition, transition probabilities per unit time due to $V_{\mathrm{SA}}$ and $V_{\mathrm{SF}}$ are investigated for the Fe ion with an anisotropy energy of $-|D| S_{Z}^{2}$, where $D$ is an anisotropy constant and $S_{Z}$ is the $Z$ component of a spin operator.
\end{abstract}

KEYWORDS: spin-atomic vibration interaction, spin-flip Hamiltonian, spin-phonon interaction, single atomic spin, spin-orbit interaction, crystal field, surface adsorbate, perturbation theory

\section{Introduction}

Recently, the magnetic properties of molecular $\operatorname{spin}^{1-9}$ and atomic $\operatorname{spin}^{10-19}$ systems have been extensively studied to develop ultimate microscopic elements for mass-storage devices and quantum information devices. In particular, the spin relaxation ${ }^{1-3,19}$ has attracted much attention from the viewpoints of data writing and storage. This spin relaxation ${ }^{20}$ means that the spin relaxes from the excited state to the ground spin state via interactions between the spin and other degrees of freedom. The degrees of freedom are considered to be, for example, the atomic and lattice vibrations. Here, the vibrational states reflect structures of the systems. Typical structures of the above atomic spin systems are a magnetic ion adsorbed on a substrate ${ }^{10}$ or the magnetic ion trapped between a tip and a substrate. ${ }^{21,22}$ For such atomic spin systems, however, theoretical studies of the interactions between the spin and vibrations have scarcely been performed so far.

A relevant interaction is considered to be spin-phonon interactions, ${ }^{23-30}$ in which a local-

*E-mail address: tskokad@ipc.shizuoka.ac.jp 
ized spin interacts with the phonon of a lattice system. The expressions of the spin-phonon interaction $^{23-29}$ have been originally derived using the spin-orbit interaction and the modulation of crystal field potential energy due to the lattice vibration. In particular, Van Vleck ${ }^{23,24}$ obtained an expression applying a perturbation theory to the following model: the crystal field potential energy in an equilibrium state was included in the unperturbed Hamiltonian, while the spin-orbit interaction and the modulation of crystal field potential energy were the perturbed one. ${ }^{29}$ Mattuck and Strandberg ${ }^{25}$ also derived essentially the same expression as Van Vleck's one ${ }^{23}$ using a slightly different method. On the other hand, Hartmann-Boutron et al. ${ }^{30}$ proposed a spin-phonon interaction applying the second-order perturbation theory to a model with the spin-orbit interaction. Here, the coefficient of the spin-phonon interaction contained the local strain on the spin site due to the phonon. ${ }^{31}$ In these spin-phonon interactions, the structure of the systems was assumed to be a periodic structure (e.g., a bulk crystal), where a unit cell consisted of the magnetic ion and the surrounding ions. ${ }^{32}$ The phonon then had a continuous energy spectrum on the assumption that the phonon frequency was proportional to the magnitude of the wave vector.

Such spin-phonon interactions, however, may not be suitable for the atomic spin on substrate (or surface), because the vibration of the adsorbate is not described in their interactions. The vibration of the adsorbate on surface is obviously different from the above-mentioned phonon. The local vibrational density of states at the adsorbate shows sharp peaks at certain energies; ${ }^{33}$ that is, this adsorbate has discrete vibrational energy levels. In contrast, the above phonon exhibits the broad vibrational density of states, ${ }^{33}$ i.e., continuous vibrational energy levels.

Furthermore, in the spin-phonon interactions, the difference in vibration displacement between an effective nucleus and electrons has not been taken into account. Here, this nucleus consists of a nucleus and core electrons. As shown in Appendix, the difference in displacement can be actually obtained, when the nucleus (plus charge) and electrons (minus charge) have the displacement in the presence of a crystalline electric field due to the surrounding ions (minus charge) (see Fig. 1). The displacements of the nucleus and the electrons are written by $\Delta r_{\mathrm{n}}$ and $(1-\eta) \Delta r_{\mathrm{n}}$, respectively, where $\eta(0 \leq \eta<1)$ is a dimensionless quantity characterizing the difference. For instance, this $\eta$ is evaluated to be about 0.05 for a single Fe ion on the $\mathrm{CuN}$ surface (see Appendix ).

On the basis of the above-mentioned facts, we aim to derive a spin-atomic vibration interaction of a single atomic spin surrounded by several ions, where the ions are present in the substrate. Here, the spin-atomic vibration interaction represents an interaction between the spin of the single magnetic ion and the vibration of this ion. This interaction also takes into account the above $\eta$.

In this paper, we derived the spin-atomic vibration interaction and a spin-flip Hamiltonian 
of a single atomic spin in a crystal field, applying a perturbation theory to a model with the spin-orbit interaction and the kinetic and potential energies of electrons. The spin-flip Hamiltonian contains spin-flip operators such as $S_{X} S_{Z}$ and $S_{Y} S_{Z}$, but no atomic vibration operators, where $S_{I}$ is the $I$ component of the spin $\boldsymbol{S}=\left(S_{X}, S_{Y}, S_{Z}\right)$. From the coefficients of the spin-atomic vibration interaction and the spin-flip Hamiltonian, we found that the former appears for $\eta \neq 0$, while the latter is present independently of $\eta$. We also obtained their coefficients in the case of the Fe ion in a crystal field of tetragonal symmetry. The magnitude of the coefficient of the spin-atomic vibration interaction was estimated to be larger than that of the conventional spin-phonon interaction in a specific region of the vibration frequency. In addition, we discussed transition probabilities per unit time due to the spin-atomic vibration interaction and spin-flip Hamiltonian of the Fe ion.

The present paper is organized as follows: In $\S 2$, we propose a model with the spin-orbit interaction and the kinetic and potential energies of electrons. This model also takes into account the vibration displacement. In $\S 3$, we derive the spin-atomic vibration interaction and spin-flip Hamiltonian. In $\S 4$, we investigate the vibration frequency dependences of their coefficients of the Fe ion in the crystal field of tetragonal symmetry. In addition, their coefficients are evaluated by assigning appropriate values to the spin-orbit coupling constant. In $\S 5$, we compare the spin-atomic vibration interaction with the conventional spin-phonon interactions. In $\S 6$, we discuss the transition probabilities per unit time due to the spin-atomic vibration interaction and the spin-flip Hamiltonian. The conclusion is presented in $§ 7$. In Appendix , we evaluate the difference in vibration displacement between the effective nucleus and the electron. The vibration displacement of the nucleus is evaluated in Appendix . In Appendix , we describe the expressions of the coefficients of the spin-atomic vibration interaction and spin-flip Hamiltonian of the Fe ion.

\section{Model}

Toward the calculation of perturbation energies in $\S 3$ (i.e., the spin-atomic vibration interaction and the spin-flip Hamiltonian), we here derive the unperturbed and perturbed Hamiltonians for a model with the spin-orbit interaction and kinetic and potential energies of electrons. This model also takes into account the difference in vibration displacement between the effective nucleus and electrons. In addition, we introduce orbital and spin states for the unperturbed Hamiltonian.

\subsection{Hamiltonian in an equilibrium state}

We first propose a Hamiltonian in an equilibrium state (with no vibration displacement) for $3 \mathrm{~d}$ electrons of an iron-group ion (i.e., a magnetic ion) in a crystal field due to the surrounding ions. Here, the $3 \mathrm{~d}$ shell of the magnetic ion is incompletely filled, and the surrounding ions have a minus charge. In particular, we treat the case of a weak crystal field. Namely, the electrons 

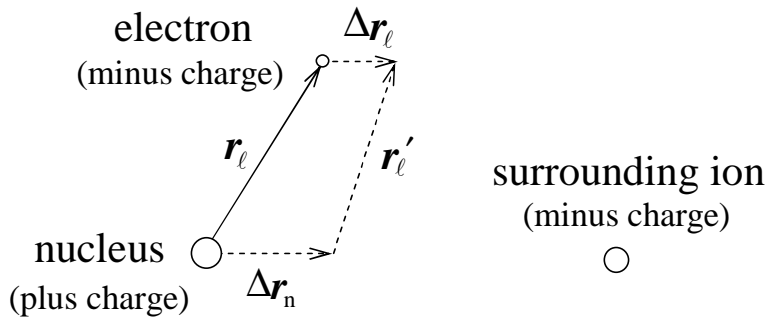

Fig. 1. Positions and displacements of the effective nucleus (the large circle) and the $\ell$ th electron (the small circle) in the single magnetic ion. The magnetic ion is surrounded by several ions. Here, one of the surrounding ions is shown by the medium circle. $\boldsymbol{r}_{\ell}$ : position vector of the $\ell$ th electron measured from the nucleus at an equilibrium position. $\Delta \boldsymbol{r}_{\mathrm{n}}$ : displacement of the nucleus. $\Delta \boldsymbol{r}_{\ell}$ : displacement of the $\ell$ th electron. $\boldsymbol{r}_{\ell}^{\prime}$ : position vector of the displaced $\ell$ th electron measured from the displaced nucleus.

occupy energy levels from the bottom keeping their spins parallel, according to Hund's first rule. ${ }^{34}$ The system is here assumed to have the total spin $\boldsymbol{S}$.

We now focus on electrons existing in the partially filled $\sigma$-spin shell of the magnetic ion, with $\sigma$ being up or down. When the number of electrons of the ground state is less than half (more than half), the $\sigma$-spin shell corresponds to the up-spin shell (down-spin shell). The excited states are then assumed to consist of orbitals in the $\sigma$-spin shell. In addition, the electrons in the $\sigma$-spin shell have the spin-orbit interaction $V_{\mathrm{so}} \cdot{ }^{35}$ Here, $\left|V_{\mathrm{so}}\right|$ is considered to be smaller than the magnitude of the crystal field potential energy. ${ }^{25}$ When the number of electrons in the $\sigma$-spin shell is $N$, we have the following Hamiltonian for the electrons:

$$
\begin{aligned}
\mathcal{H} & =\mathcal{H}_{\mathrm{e}}+V_{\mathrm{so}}, \\
\mathcal{H}_{\mathrm{e}} & =\sum_{\ell=1}^{N}\left[\frac{\boldsymbol{p}_{\ell}^{2}}{2 m}+V_{\mathrm{en}}\left(\boldsymbol{r}_{\ell}\right)+V_{\mathrm{c}}\left(\boldsymbol{r}_{\ell}\right)\right], \\
V_{\mathrm{SO}} & =\lambda \boldsymbol{L} \cdot \boldsymbol{S},
\end{aligned}
$$

with

$$
\begin{aligned}
& \boldsymbol{p}_{\ell}=\left(p_{\ell, x}, p_{\ell, y}, p_{\ell, z}\right)=m \dot{\boldsymbol{r}}_{\ell}, \\
& V_{\mathrm{en}}\left(\boldsymbol{r}_{\ell}\right)=-\frac{Z_{\mathrm{eff}} e^{2}}{4 \pi \epsilon_{0} r_{\ell}}, \\
& V_{\mathrm{c}}\left(\boldsymbol{r}_{\ell}\right)=\sum_{\ell^{\prime}} \frac{Z_{\ell^{\prime}} e^{2}}{4 \pi \epsilon_{0}\left|\boldsymbol{r}_{\ell}-\boldsymbol{R}_{\ell^{\prime}}\right|}, \\
& \lambda=C_{ \pm}\left\langle\frac{1}{r^{3}}\right\rangle, \\
& \left\langle\frac{1}{r^{3}}\right\rangle=\sum_{\ell=1}^{N}\left(\Psi_{1}\left|r_{\ell}^{-3}\right| \Psi_{1}\right) / \sum_{\ell=1}^{N} 1,
\end{aligned}
$$




$$
\boldsymbol{L}=\left(L_{x}, L_{y}, L_{z}\right)=\frac{1}{\hbar} \sum_{\ell=1}^{N}\left(\boldsymbol{r}_{\ell} \times \boldsymbol{p}_{\ell}\right),
$$

with $\boldsymbol{r}_{\ell}=\left(x_{\ell}, y_{\ell}, z_{\ell}\right), r_{\ell}=\left|\boldsymbol{r}_{\ell}\right|=\sqrt{x_{\ell}^{2}+y_{\ell}^{2}+z_{\ell}^{2}}, \boldsymbol{S}=\left(S_{x}, S_{y}, S_{z}\right), C_{ \pm}= \pm\left(\mu_{0} / 4 \pi\right) g \mu_{\mathrm{B}}^{2} Z_{\mathrm{eff}} / 2 S$, and $\mu_{\mathrm{B}}=e \hbar / 2 m$. Here, $\mathcal{H}_{\mathrm{e}}$ denotes the Hamiltonian consisting of the kinetic energy $\sum_{\ell=1}^{N} \boldsymbol{p}_{\ell}^{2} /(2 m)$ and the potential energy $\sum_{\ell=1}^{N}\left[V_{\mathrm{en}}\left(\boldsymbol{r}_{\ell}\right)+V_{\mathrm{c}}\left(\boldsymbol{r}_{\ell}\right)\right]$. The quantity $m$ is the electron mass, $\boldsymbol{r}_{\ell}$ is the position of the $\ell$ th electron measured from the nucleus (see Fig. 1), and $\boldsymbol{p}_{\ell}$ is the momentum of the $\ell$ th electron. The operator $V_{\mathrm{en}}\left(\boldsymbol{r}_{\ell}\right)$ is a spherically symmetric potential energy of the $\ell$ th electron created by a nucleus and the other electrons in the magnetic ion. The quantity $-e(<0)$ is the electronic charge, and $\epsilon_{0}$ is the electric constant. In addition, $Z_{\text {eff }}$ represents the effective nuclear charge for the $3 \mathrm{~d}$ electron, ${ }^{36}$ which can be evaluated from Slater's rule. ${ }^{37}$ The operator $V_{\mathrm{c}}\left(\boldsymbol{r}_{\ell}\right)$ is the crystal field potential energy, where $\boldsymbol{R}_{\ell^{\prime}}$ and $-Z_{\ell^{\prime}} e(<0)$ are the position vector and the electric charge of the $\ell^{\prime}$ th surrounding ion, respectively. As to $\lambda$ of eq. (7), $C_{+}\left(C_{-}\right)$is the coefficient in the case of an electron number of less than half (more than half) in an incompletely filled $3 \mathrm{~d}$ shell. ${ }^{35}$ The mean $\left\langle r^{-3}\right\rangle$ is defined by eq. (8), where $\left.\mid \Psi_{1}\right)$ is the orbital state of the electrons in the $\sigma$-spin shell of the ground state (see eq. (41)). The quantity $\mu_{\mathrm{B}}$ is the Bohr magneton, $g$ is the $\mathrm{g}$ value, $\mu_{0}$ is the permeability, and $\hbar$ is the Planck constant $h$ divided by $2 \pi$. Also, $S$ is the magnitude of $\boldsymbol{S}$, and $\hbar \boldsymbol{L}$ is the total orbital angular momentum.

\subsection{Vibration displacement}

For the Hamiltonian in the equilibrium state of $\S 2.1$, we take the effect of the vibration displacement into consideration. Regarding the vibration, we consider a simple model, in which only a single magnetic ion vibrates, while the surrounding ions are rigid. This model may be relevant to, for example, a single magnetic ion surrounded by several ions in the substrate. Here, the surrounding ions are assumed to behave as an oscillator with the magnitude of vibration displacement, which is much smaller than that of the magnetic ion. This assumption is justified under the following conditions: (i) The coupling within the surrounding ions is stronger than that between the magnetic ion and the surrounding ions. (ii) The total mass of the surrounding ions is sufficiently larger than the mass of the magnetic ion. ${ }^{38,39}$ For example, in the case of the Fe ion on the CuN surface, ${ }^{10}$ the Fe ion is surrounded by two $\mathrm{N}$ ions and five $\mathrm{Cu}$ ions. The total mass of the surrounding ions is more than six times as large as the mass of the Fe ion. (iii) A force constant between the surrounding ions and the other ions adjacent to them in the substrate is much larger than that between the surrounding ions and the magnetic ion. ${ }^{38}$

We now focus on the effective nucleus of the magnetic ion. Here, the nucleus consists of a nucleus and core electrons, where the core electrons correspond to the electrons other than those in the $\sigma$-spin shell. The effective nucleus is first assumed to have a slight vibration 
displacement, $\Delta \boldsymbol{r}_{\mathrm{n}}$ (see Fig. 1 and Appendix ), i.e.,

$$
\Delta \boldsymbol{r}_{\mathrm{n}}=\left(\Delta x_{\mathrm{n}}, \Delta y_{\mathrm{n}}, \Delta z_{\mathrm{n}}\right)
$$

The displacement of the $\ell$ th electron in the $\sigma$-spin shell, $\Delta \boldsymbol{r}_{\ell}$, is then given by

$$
\Delta \boldsymbol{r}_{\ell}=(1-\eta) \Delta \boldsymbol{r}_{\mathrm{n}}
$$

where $-\eta \Delta \boldsymbol{r}_{\mathrm{n}}$ represents the difference in displacement between the nucleus and the electron. This $-\eta \Delta \boldsymbol{r}_{\mathrm{n}}$ appears in the presence of the crystalline electric field due to the surrounding ions (see Appendix ). Here, the dimensionless quantity characterizing the difference $\eta(0 \leq \eta<1)$ has been assumed to be independent of $\ell$ and $\Delta \boldsymbol{r}_{\mathrm{n}}$ for simplicity. For example, this $\eta$ may be written by eq. (A.8). Regarding eq. (11), we have considered the following situation: First, the effective nucleus of the magnetic ion has the displacement of $\Delta \boldsymbol{r}_{\mathrm{n}}$. Second, each electron moves in a circular orbit around "the nucleus fixed at $\Delta \boldsymbol{r}_{\mathrm{n}}$ " at a high speed, and simultaneously experiences the crystalline electric field due to the surrounding ions. This electric field shifts the center position of the circular orbit from $\Delta \boldsymbol{r}_{\mathrm{n}}$ to $(1-\eta) \Delta \boldsymbol{r}_{\mathrm{n}}$ (see Appendix ).

Thus, when the nucleus has the displacement of $\Delta \boldsymbol{r}_{\mathrm{n}}$, the position of the $\ell$ th electron measured from the nucleus, $\boldsymbol{r}_{\ell}^{\prime}$, is represented by

$$
\boldsymbol{r}_{\ell}^{\prime}=\boldsymbol{r}_{\ell}+\Delta \boldsymbol{r}_{\ell}-\Delta \boldsymbol{r}_{\mathrm{n}}=\boldsymbol{r}_{\ell}-\eta \Delta \boldsymbol{r}_{\mathrm{n}}
$$

From the time differential of eq. (12), we obtain the following momentum:

$$
\boldsymbol{p}_{\ell}^{\prime}=\boldsymbol{p}_{\ell}-\eta \Delta \boldsymbol{p}_{\mathrm{e}}
$$

with

$$
\begin{aligned}
& \boldsymbol{p}_{\ell}^{\prime}=m \dot{\boldsymbol{r}}_{\ell}^{\prime}, \\
& \Delta \boldsymbol{p}_{\mathrm{e}}=\left(\Delta p_{\mathrm{e}, x}, \Delta p_{\mathrm{e}, y}, \Delta p_{\mathrm{e}, z}\right)=m \Delta \dot{\boldsymbol{r}}_{\mathrm{n}} .
\end{aligned}
$$

\subsection{Unperturbed and perturbed Hamiltonians}

We next derive the unperturbed and perturbed Hamiltonians from a Hamiltonian, $\mathcal{H}^{\text {disp }}$, which contains the effect of the above-mentioned vibration displacement. This $\mathcal{H}^{\text {disp }}$ can be obtained on the basis of $\mathcal{H}$ of eq. (1). Namely, when $\boldsymbol{r}_{\ell}^{\prime}$ of eq. (12) and $\boldsymbol{p}_{\ell}^{\prime}$ of eq. (13) are taken into account, $\mathcal{H}$ is rewritten as

$$
\begin{aligned}
\mathcal{H}^{\text {disp }} & =\mathcal{H}_{\mathrm{e}}^{\text {disp }}+V_{\mathrm{SO}}^{\text {disp }}, \\
\mathcal{H}_{\mathrm{e}}^{\text {disp }} & =\sum_{\ell=1}^{N}\left[\frac{\boldsymbol{p}_{\ell}^{\prime 2}}{2 m}+V_{\mathrm{en}}\left(\boldsymbol{r}_{\ell}^{\prime}\right)+V_{\mathrm{c}}\left(\boldsymbol{r}_{\ell}^{\prime}+\Delta \boldsymbol{r}_{\mathrm{n}}\right)\right], \\
V_{\mathrm{SO}}^{\text {disp }} & =C_{ \pm}\left\langle\frac{1}{{r^{\prime}}^{3}}\right\rangle \sum_{\ell=1}^{N}\left(\frac{\boldsymbol{r}_{\ell}^{\prime} \times \boldsymbol{p}_{\ell}^{\prime}}{\hbar}\right) \cdot \boldsymbol{S}
\end{aligned}
$$


where

$$
\begin{aligned}
& \left\langle\frac{1}{r^{\prime 3}}\right\rangle=\sum_{\ell=1}^{N}\left(\Psi_{1}\left|r_{\ell}^{\prime-3}\right| \Psi_{1}\right) / \sum_{\ell=1}^{N} 1 \\
& r_{\ell}^{\prime}=\sqrt{\left(x_{\ell}-\eta \Delta x_{\mathrm{n}}\right)^{2}+\left(y_{\ell}-\eta \Delta y_{\mathrm{n}}\right)^{2}+\left(z_{\ell}-\eta \Delta z_{\mathrm{n}}\right)^{2}} \\
& \boldsymbol{r}_{\ell}^{\prime}+\Delta \boldsymbol{r}_{\mathrm{n}}=\boldsymbol{r}_{\ell}+(1-\eta) \Delta \boldsymbol{r}_{\mathrm{n}} .
\end{aligned}
$$

For eq. (16), we expand $V_{\mathrm{en}}\left(\boldsymbol{r}_{\ell}^{\prime}\right)=V_{\mathrm{en}}\left(\boldsymbol{r}_{\ell}-\eta \Delta \boldsymbol{r}_{\mathrm{n}}\right)$ and $V_{\mathrm{c}}\left(\boldsymbol{r}_{\ell}^{\prime}+\Delta \boldsymbol{r}_{\mathrm{n}}\right)=V_{\mathrm{c}}\left(\boldsymbol{r}_{\ell}+(1-\eta) \Delta \boldsymbol{r}_{\mathrm{n}}\right)$ in power series in $\eta \Delta \xi_{\mathrm{n}}$ and $(1-\eta) \Delta \xi_{\mathrm{n}}$, respectively, with $\xi=x, y, z$. In addition, $\left\langle r^{\prime-3}\right\rangle$ of $V_{\mathrm{SO}}^{\text {disp }}$ is expanded in power series in $\eta \Delta \xi_{\mathrm{n}}$. Since $\left|\eta \Delta \xi_{\mathrm{n}}\right|,\left|(1-\eta) \Delta \xi_{\mathrm{n}}\right|$, and $\left|\eta \Delta p_{\mathrm{e}, \xi}\right|$ are considered to be sufficiently small, we neglect terms higher than the first order of $\eta \Delta \xi_{\mathrm{n}},(1-\eta) \Delta \xi_{\mathrm{n}}$, and $\eta \Delta p_{\mathrm{e}, \xi}$

For such $\mathcal{H}^{\text {disp}}$, we adopt Van Vleck's approach; ${ }^{23}$ that is, the crystal field potential energy in the equilibrium state is contained in the unperturbed Hamiltonian, while the spin-orbit interaction and the modulation of crystal field potential energy due to the vibration displacement are treated as perturbations. We therefore present the following effective Hamiltonian consisting of the unperturbed term $\mathcal{H}_{0}$ and the perturbed term $V_{\mathrm{p}}$ :

$$
\begin{aligned}
& \mathcal{H}_{\mathrm{eff}}=\mathcal{H}_{0}+V_{\mathrm{p}} \\
& V_{\mathrm{p}}=V_{\mathrm{k}}^{\prime}+V_{\mathrm{en}}^{\prime}+V_{\mathrm{c}}^{\prime}+V_{\mathrm{so}} \\
& V_{\mathrm{so}}=V_{\mathrm{so} 0}+V_{\mathrm{so} 1}+V_{\mathrm{so} 2}
\end{aligned}
$$

with

$$
\begin{aligned}
& \mathcal{H}_{0}=\sum_{\ell=1}^{N}\left[\frac{\boldsymbol{p}_{\ell}^{2}}{2 m}+V_{\mathrm{en}}\left(\boldsymbol{r}_{\ell}\right)+V_{\mathrm{c}}\left(\boldsymbol{r}_{\ell}\right)\right] \\
& V_{\mathrm{k}}^{\prime}=-\frac{\eta}{m} \boldsymbol{p}_{t} \cdot \Delta \boldsymbol{p}_{\mathrm{e}} \\
& V_{\mathrm{en}}^{\prime}=-\eta \sum_{\ell=1}^{N}\left(\Delta x_{\mathrm{n}} \frac{\partial}{\partial x_{\ell}}+\Delta y_{\mathrm{n}} \frac{\partial}{\partial y_{\ell}}+\Delta z_{\mathrm{n}} \frac{\partial}{\partial z_{\ell}}\right) V_{\mathrm{en}}\left(\boldsymbol{r}_{\ell}\right), \\
& V_{\mathrm{c}}^{\prime}=(1-\eta) \sum_{\ell=1}^{N}\left(\Delta x_{\mathrm{n}} \frac{\partial}{\partial x_{\ell}}+\Delta y_{\mathrm{n}} \frac{\partial}{\partial y_{\ell}}+\Delta z_{\mathrm{n}} \frac{\partial}{\partial z_{\ell}}\right) V_{\mathrm{c}}\left(\boldsymbol{r}_{\ell}\right), \\
& V_{\mathrm{so} 0}=\lambda \boldsymbol{L} \cdot \boldsymbol{S}, \\
& V_{\mathrm{so} 1}=\eta C_{ \pm}\left\langle\frac{3 x}{r^{5}} \Delta x_{\mathrm{n}}+\frac{3 y}{r^{5}} \Delta y_{\mathrm{n}}+\frac{3 z}{r^{5}} \Delta z_{\mathrm{n}}\right\rangle \boldsymbol{L} \cdot \boldsymbol{S}, \\
& V_{\mathrm{so} 2}=-\eta \lambda \frac{1}{\hbar}\left(\boldsymbol{r}_{t} \times \Delta \boldsymbol{p}_{\mathrm{e}}+\Delta \boldsymbol{r}_{\mathrm{n}} \times \boldsymbol{p}_{t}\right) \cdot \boldsymbol{S},
\end{aligned}
$$

where

$$
\boldsymbol{r}_{t}=\left(x_{t}, y_{t}, z_{t}\right)=\sum_{\ell=1}^{N} \boldsymbol{r}_{\ell}
$$


J. Phys. Soc. Jpn.

Full PAPER

$$
\begin{aligned}
& \boldsymbol{p}_{t}=\left(p_{t, x}, p_{t, y}, p_{t, z}\right)=\sum_{\ell=1}^{N} \boldsymbol{p}_{\ell}, \\
& \left\langle\frac{3 \xi}{r^{5}} \Delta \xi_{\mathrm{n}}\right\rangle=\sum_{\ell=1}^{N}\left(\Psi_{1}\left|3 \xi_{\ell} r_{\ell}^{-5} \Delta \xi_{\mathrm{n}}\right| \Psi_{1}\right) / \sum_{\ell=1}^{N} 1,
\end{aligned}
$$

for $\xi=x, y, z$. Here, eq. (25) is the zeroth-order term of $\eta \Delta p_{\mathrm{e}, \xi}, \eta \Delta \xi_{\mathrm{n}}$, and $(1-\eta) \Delta \xi_{\mathrm{n}}$ in eq. (17). Equation (26) is the first-order term of $\eta \Delta \boldsymbol{p}_{\mathrm{e}}$ in $\sum_{\ell=1}^{N} \boldsymbol{p}_{\ell}^{\prime 2} / 2 m$, while eq. (27) is that of $\eta \Delta \xi_{\mathrm{n}}$ in $\sum_{\ell=1}^{N} V_{\mathrm{en}}\left(\boldsymbol{r}_{\ell}^{\prime}\right)$. Equation (28) is the first-order term of $(1-\eta) \Delta \xi_{\mathrm{n}}$ in $\sum_{\ell=1}^{N} V_{\mathrm{c}}\left(\boldsymbol{r}_{\ell}+(1-\eta) \Delta \boldsymbol{r}_{\mathrm{n}}\right)$. In addition, eq. (29) is the zeroth-order term of $\eta \Delta \xi_{\mathrm{n}}$ and $\eta \Delta p_{\mathrm{e}, \xi}$ in eq. (18), while eqs. (30) and (31) are the first-order terms of them in eq. (18). Here, eq. (30) (eq. (31)) corresponds to a term with a change of $\lambda$ due to the vibration (a term with a change of $\boldsymbol{L}$ due to the vibration). Note that $\Delta x_{\mathrm{n}}, \Delta y_{\mathrm{n}}$, and $\Delta z_{\mathrm{n}}$ in \langle\rangle of eq. (30) can be put outside of \langle\rangle , which is related to electron systems. Also, the suffix $t$ in eqs. (32) and (33) represents the total sum.

\subsection{Orbital and spin states}

For the calculation of the perturbation energies, we here propose a specific orbital and spin state for $\mathcal{H}_{0}$ of eq. (25). Simultaneously, we introduce a $3 \mathrm{~d}$ orbital state that can be utilized in the principal axis transformation of $\S 3.3$.

On the basis of eq. (25), we first have the $3 \mathrm{~d}$ orbital and spin state with the largest $S$, i.e.,

$$
\left|\Phi_{j}\right\rangle\left|S_{z}\right\rangle
$$

where $\left|S_{z}\right\rangle$ is a spin state with a $(2 S+1)$-fold degeneracy of $S_{z}=-S-S$, and $\left|\Phi_{j}\right\rangle$ is an orbital state of the electrons in the partially filled $\sigma$-spin shell. When the number of the electrons is $N$, the wave function for $\left|\Phi_{j}\right\rangle, \Phi_{j}$, is given by the following Slater determinant:

$$
\Phi_{j}=\frac{1}{\sqrt{N !}}\left|\begin{array}{cccc}
d_{\alpha}(1) & d_{\alpha}(2) & \cdots & d_{\alpha}(N) \\
d_{\beta}(1) & d_{\beta}(2) & \cdots & d_{\beta}(N) \\
\vdots & & \ddots & \vdots \\
d_{\gamma}(1) & d_{\gamma}(2) & \cdots & d_{\gamma}(N)
\end{array}\right|
$$

The suffix $j$ of $\Phi_{j}$ specifies the electron configuration, in which the occupied orbitals are represented by $\alpha, \beta, \cdots$, and $\gamma$. The function $d_{\zeta}(\ell)$ represents the $3 \mathrm{~d}$ orbital of the $\sigma$-spin, where $\ell=1,2, \cdots, N$ and $\zeta=\alpha, \beta, \cdots$, and $\gamma$. We also have the following eigenvalue equation:

$$
\mathcal{H}_{0}\left|\Phi_{j}\right\rangle=e_{j}\left|\Phi_{j}\right\rangle
$$

with

$$
\begin{aligned}
& e_{j}=\varepsilon_{d_{\alpha}}+\varepsilon_{d_{\beta}}+\cdots+\varepsilon_{d_{\gamma}}=\sum_{\zeta \in j} \varepsilon_{d_{\zeta}}, \\
& {\left[-\frac{\hbar^{2}}{2 m} \nabla_{\ell}^{2}+V_{\mathrm{en}}\left(\boldsymbol{r}_{\ell}\right)+V_{\mathrm{c}}\left(\boldsymbol{r}_{\ell}\right)\right] d_{\zeta}(\ell)=\varepsilon_{d_{\zeta}} d_{\zeta}(\ell),}
\end{aligned}
$$


J. Phys. Soc. Jpn.

Full PAPER

where $e_{j}$ is the eigenenergy for $\left|\Phi_{j}\right\rangle$, and $\varepsilon_{d_{\zeta}}$ is that for $d_{\zeta}(\ell)$. In addition, $\left|\Phi_{1}\right\rangle$ is assumed to be a nondegenerate ground state with $e_{1}$. Since $\mathcal{H}_{0}$ is a real function, $\Phi_{1}$ can be a real one. ${ }^{40}$ This $\left|\Phi_{j}\right\rangle$ will be used to perform the principal axis transformation as described in $§ 3.3$.

Second, we propose the orbital and spin state to obtain the perturbation energy (i.e., the spin-atomic vibration interaction and the spin-flip Hamiltonian); that is,

$$
\left.\mid \Psi_{j}\right)\left|S_{z}\right\rangle
$$

where $\left|S_{z}\right\rangle$ is the above-mentioned spin state, and $\left.\mid \Psi_{j}\right)$ is an orbital state of the electrons in the $\sigma$-spin shell. The wave function for $\left.\mid \Psi_{j}\right), \Psi_{j}$, is assumed to be

$$
\Psi_{j}=\frac{1}{\sqrt{N !}}\left|\begin{array}{cccc}
\psi_{\alpha}(1) & \psi_{\alpha}(2) & \cdots & \psi_{\alpha}(N) \\
\psi_{\beta}(1) & \psi_{\beta}(2) & \cdots & \psi_{\beta}(N) \\
\vdots & & \ddots & \vdots \\
\psi_{\gamma}(1) & \psi_{\gamma}(2) & \cdots & \psi_{\gamma}(N)
\end{array}\right|
$$

with

$$
\begin{aligned}
& \psi_{\zeta}(\ell)=C_{\zeta}\left(d_{\zeta}(\ell)+\sum_{m=1(\neq \zeta)}^{5} c_{d_{m}}^{(\zeta)} \overline{d_{m}}(\ell)+\sum_{n=1}^{3} c_{p_{n}}^{(\zeta)} \overline{p_{n}}(\ell)\right) \\
& C_{\zeta}=\left(1+\sum_{m=1(\neq \zeta)}^{5}\left|c_{d_{m}}^{(\zeta)}\right|^{2}+\sum_{n=1}^{3}\left|c_{p_{n}}^{(\zeta)}\right|^{2}\right)^{-1 / 2},
\end{aligned}
$$

where $N$ is the number of the electrons. The suffix $j$ of $\Psi_{j}$ specifies the electron configuration, in which the occupied orbitals are represented by $\alpha, \beta, \cdots$, and $\gamma$. The function $d_{\zeta}(\ell)$ is the dominant $3 \mathrm{~d}$ orbital of the $\sigma$-spin, while $\overline{d_{m}}(\ell)$ and $\overline{p_{n}}(\ell)$ are the other $3 \mathrm{~d}$ orbital of the $\sigma$-spin and the $4 \mathrm{p}$ orbital of the $\sigma$-spin in the same magnetic ion, respectively. Owing to the d-d and d-p hybridizations in the magnetic ion, $\overline{d_{m}}(\ell)$ and $\overline{p_{n}}(\ell)$ are included in eq. (42). The hybridization originate from, for example, the mixing of atomic orbitals via the surrounding ions. The bars of $\overline{d_{m}}(\ell)$ and $\overline{p_{n}}(\ell)$ are a mark to distinguish the components hybridized to $d_{\zeta}(\ell)$. The quantities $c_{d_{m}}^{(\zeta)}$ and $c_{p_{n}}^{(\zeta)}$ are the coefficients for $\overline{d_{m}}(\ell)$ and $\overline{p_{n}}(\ell)$, respectively, and $C_{\zeta}$ is the normalization factor, where $\left|c_{d_{m}}^{(\zeta)}\right|^{2} \ll 1$ and $\left|c_{p_{n}}^{(\zeta)}\right|^{2} \ll 1$ are assumed. Note that $\overline{d_{m}}(\ell)$ and $\overline{p_{n}}(\ell)$ play important roles in the presence of the spin-atomic vibration interaction, as will be described later.

We will also use the energy of $\left.\mid \Psi_{j}\right)$ in the perturbation calculation. For simplicity, the energy is assumed to be

$$
E_{j}=\left(\Psi_{j}\left|\mathcal{H}_{0}\right| \Psi_{j}\right)
$$

where it is noted that $\mathcal{H}_{0}$ of eq. (25) (or eq. (2)) is the Hamiltonian in the subspace of the $3 \mathrm{~d}$ orbitals. We here write $E_{j}$ as

$$
E_{j}=e_{j}+\Delta e_{j}
$$


J. Phys. Soc. Jpn.

Full PAPER

where $e_{j}$ is eq. (38), while $\Delta e_{j}$ is given by $E_{j}-e_{j}$. This $\Delta e_{j}$ consists of terms higher than the first order of $c_{d_{m}}^{(\zeta)}$ and $c_{p_{n}}^{(\zeta)}$, where $\left|\Delta e_{i}-\Delta e_{j}\right| \ll\left|e_{i}-e_{j}\right|$ for $i \neq j$.

In this paper, eq. (44) is simultaneously regarded as the matrix element of the effective Hamiltonian of $\mathcal{H}_{0}$. Namely, we neglect the off-diagonal matrix element $\left(\Psi_{j^{\prime}}\left|\mathcal{H}_{0}\right| \Psi_{j}\right)$ for $j \neq j^{\prime}$, which appears due to the hybridization, because the magnitudes of the off-diagonal elements are much smaller than $\left|E_{j}-E_{j^{\prime}}\right|$. The effective Hamiltonian will be used in eqs. (59) and (60).

Furthermore, $\left.\mid \Psi_{1}\right)$ is assumed to be a nondegenerate ground state with $E_{1}$. The function $\Psi_{1}$ is regarded as a real function because $\mathcal{H}_{0}$ is the real Hamiltonian. ${ }^{40}$

\section{Spin-Atomic Vibration Interaction and Spin-Flip Hamiltonian}

We obtain the first- and second-order perturbation energies of the model in $\S 2$. By means of the principal axis transformation, we finally derive the spin-atomic vibration interaction and the spin-flip Hamiltonian as well as the conventional anisotropy spin Hamiltonian.

\subsection{First-order perturbation energy}

Using $V_{\mathrm{p}}$ of eq. (23) and $\left.\mid \Psi_{j}\right)\left|S_{z}\right\rangle$ of eq. (40), we obtain the first-order perturbation energy $V^{(1)}$, i.e.,

$$
V^{(1)}=\left\langle S_{z}{ }^{\prime}\left|\left(\Psi_{1}\left|V_{\mathrm{p}}\right| \Psi_{1}\right)\right| S_{z}\right\rangle .
$$

We here have $\left(\Psi_{1}\left|V_{\mathrm{so0}}^{(1)}\right| \Psi_{1}\right)=\left(\Psi_{1}\left|V_{\mathrm{so} 1}^{(1)}\right| \Psi_{1}\right)=0$, because $\boldsymbol{L}$ is quenched for the nondegenerate ground state whose wave function is the real function, i.e., $\left(\Psi_{1}|\boldsymbol{L}| \Psi_{1}\right)=0 .{ }^{40-42}$ The first-order energy $V^{(1)}$ is then expressed as follows:

$$
\begin{aligned}
V^{(1)} & =V_{\mathrm{so} 2}^{(1)}+V_{\mathrm{en}}^{(1)}+V_{\mathrm{c}}^{(1)}+V_{\mathrm{k}}^{(1)} \\
V_{\mathrm{so} 2}^{(1)} & =\left\langle S_{z}{ }^{\prime}\left|\left(\Psi_{1}\left|V_{\mathrm{so} 2}\right| \Psi_{1}\right)\right| S_{z}\right\rangle=\sum_{\mu=x, y, z} \Pi_{\mu} S_{\mu} \\
V_{\mathrm{en}}^{(1)} & =\left\langle S_{z}{ }^{\prime}\left|\left(\Psi_{1}\left|V_{\mathrm{en}}^{\prime}\right| \Psi_{1}\right)\right| S_{z}\right\rangle \\
& =-\eta \sum_{\xi=x, y, z}\left(\Psi_{1}\left|\sum_{\ell=1}^{N} \frac{\partial}{\partial \xi_{\ell}} V_{\mathrm{en}}\left(\boldsymbol{r}_{\ell}\right)\right| \Psi_{1}\right) \sqrt{\frac{\hbar}{2 M \omega_{\xi}}}\left(a_{\xi}+a_{\xi}^{\dagger}\right) \delta_{S_{z^{\prime}}, S_{z}}, \\
V_{\mathrm{c}}^{(1)} & =\left\langle S_{z}{ }^{\prime}\left|\left(\Psi_{1}\left|V_{\mathrm{c}}^{\prime}\right| \Psi_{1}\right)\right| S_{z}\right\rangle \\
& =(1-\eta) \sum_{\xi=x, y, z}\left(\Psi_{1}\left|\sum_{\ell=1}^{N} \frac{\partial}{\partial \xi_{\ell}} V_{\mathrm{c}}\left(\boldsymbol{r}_{\ell}\right)\right| \Psi_{1}\right) \sqrt{\frac{\hbar}{2 M \omega_{\xi}}}\left(a_{\xi}+a_{\xi}^{\dagger}\right) \delta_{S_{z^{\prime}}, S_{z}}, \\
V_{\mathrm{k}}^{(1)} & =\left\langle S_{z}{ }^{\prime}\left|\left(\Psi_{1}\left|V_{\mathrm{k}}^{\prime}\right| \Psi_{1}\right)\right| S_{z}\right\rangle \\
& =-\frac{\eta}{M} \sum_{\xi=x, y, z}\left(\Psi_{1}\left|p_{t, \xi}\right| \Psi_{1}\right) \mathrm{i} \sqrt{\frac{M \hbar \omega_{\xi}}{2}}\left(-a_{\xi}+a_{\xi}^{\dagger}\right) \delta_{S_{z}{ }^{\prime}, S_{z}} \\
& =0,
\end{aligned}
$$


with

$$
\begin{aligned}
\Pi_{x} & =-\eta \lambda \frac{1}{\hbar}\left[\left(\Psi_{1}\left|y_{t}\right| \Psi_{1}\right) \Delta p_{\mathrm{e}, z}-\left(\Psi_{1}\left|z_{t}\right| \Psi_{1}\right) \Delta p_{\mathrm{e}, y}\right] \\
& =\Lambda_{y, z}^{(1)}\left(-a_{z}+a_{z}^{\dagger}\right)-\Lambda_{z, y}^{(1)}\left(-a_{y}+a_{y}^{\dagger}\right), \\
\Pi_{y} & =-\eta \lambda \frac{1}{\hbar}\left[\left(\Psi_{1}\left|z_{t}\right| \Psi_{1}\right) \Delta p_{\mathrm{e}, x}-\left(\Psi_{1}\left|x_{t}\right| \Psi_{1}\right) \Delta p_{\mathrm{e}, z}\right] \\
& =\Lambda_{z, x}^{(1)}\left(-a_{x}+a_{x}^{\dagger}\right)-\Lambda_{x, z}^{(1)}\left(-a_{z}+a_{z}^{\dagger}\right), \\
\Pi_{z} & =-\eta \lambda \frac{1}{\hbar}\left[\left(\Psi_{1}\left|x_{t}\right| \Psi_{1}\right) \Delta p_{\mathrm{e}, y}-\left(\Psi_{1}\left|y_{t}\right| \Psi_{1}\right) \Delta p_{\mathrm{e}, x}\right] \\
& =\Lambda_{x, y}^{(1)}\left(-a_{y}+a_{y}^{\dagger}\right)-\Lambda_{y, x}^{(1)}\left(-a_{x}+a_{x}^{\dagger}\right),
\end{aligned}
$$

where

$$
\Lambda_{\mu, \xi}^{(1)}=-\eta \lambda \frac{1}{\hbar} \mathrm{i} \frac{m}{M} \sqrt{\frac{M \hbar \omega_{\xi}}{2}}\left(\Psi_{1}\left|\mu_{t}\right| \Psi_{1}\right) .
$$

In the last result of eq. (48), $\left\langle S_{z}^{\prime}\right|$ and $\left|S_{z}\right\rangle$ are omitted to use the operator representation. The vibration displacement of the effective nucleus, $\Delta \boldsymbol{r}_{\mathrm{n}}$ of eq. (10), has been replaced here with

$$
\begin{aligned}
\Delta \boldsymbol{r}_{\mathrm{n}} & =\left(\Delta x_{\mathrm{n}}, \Delta y_{\mathrm{n}}, \Delta z_{\mathrm{n}}\right) \\
& =\left(\sqrt{\frac{\hbar}{2 M \omega_{x}}}\left(a_{x}+a_{x}^{\dagger}\right), \sqrt{\frac{\hbar}{2 M \omega_{y}}}\left(a_{y}+a_{y}^{\dagger}\right), \sqrt{\frac{\hbar}{2 M \omega_{z}}}\left(a_{z}+a_{z}^{\dagger}\right)\right) .
\end{aligned}
$$

The operator $a_{\xi}\left(a_{\xi}^{\dagger}\right)$ denotes the annihilation operator (creation operator) of the atomic vibration in the $\xi$ direction, ${ }^{30}$ where the atomic vibration represents the vibration of the magnetic ion. The quantity $M$ is the mass of the magnetic ion, and $\omega_{\xi}$ is the angular frequency in the $\xi$ direction of the magnetic ion. On the basis of eq. (15), $\Delta p_{\mathrm{e}}$ has been written as

$$
\Delta \boldsymbol{p}_{\mathrm{e}}=m \Delta \dot{\boldsymbol{r}}_{\mathrm{n}}=m \frac{\Delta \boldsymbol{p}_{\mathrm{n}}}{M},
$$

where $\Delta \boldsymbol{p}_{\mathrm{n}}$ is the momentum of the effective nucleus. This $\Delta \boldsymbol{p}_{\mathrm{n}}$ also has been replaced with

$$
\begin{aligned}
\Delta \boldsymbol{p}_{\mathrm{n}} & =\left(\Delta p_{\mathrm{n}, x}, \Delta p_{\mathrm{n}, y}, \Delta p_{\mathrm{n}, z}\right) \\
& =\left(\mathrm{i} \sqrt{\frac{M \hbar \omega_{x}}{2}}\left(-a_{x}+a_{x}^{\dagger}\right), \mathrm{i} \sqrt{\frac{M \hbar \omega_{y}}{2}}\left(-a_{y}+a_{y}^{\dagger}\right), \mathrm{i} \sqrt{\frac{M \hbar \omega_{z}}{2}}\left(-a_{z}+a_{z}^{\dagger}\right)\right) .
\end{aligned}
$$

Note here that $\Delta \boldsymbol{r}_{\mathrm{n}}$ (i.e., the displacement of the effective nucleus) may correspond to the position of the gravity point of the magnetic ion, because the mass of the nucleus is considerably larger than that of the electron. In addition, $\Delta \boldsymbol{r}_{\mathrm{n}}\left(\Delta \boldsymbol{p}_{\mathrm{n}}\right)$ has been assumed to be equal to the vibration displacement (momentum) of a mass point acting as a harmonic oscillator, in which a restoring force is linear in the displacement (see eq. (A.1) and Appendix ). The mass of the mass point is $M$, and its angular frequency in the $\xi$ direction is $\omega_{\xi}$.

In the derivation of eqs. (51) - (54), we have used $\left(\Psi_{1}\left|p_{t, \mu}\right| \Psi_{1}\right)=0$ with $\mu=x, y, z$. Here, 
$\left(\Psi_{j^{\prime}}\left|p_{t, \mu}\right| \Psi_{j}\right)$ is represented by

$$
\begin{aligned}
\left(\Psi_{j^{\prime}}\left|p_{t, \mu}\right| \Psi_{j}\right) & =\frac{m}{\mathrm{i} \hbar}\left(\Psi_{j^{\prime}}\left|\left[\mu_{t}, \mathcal{H}_{0}\right]\right| \Psi_{j}\right) \\
& =\frac{m\left(E_{j}-E_{j^{\prime}}\right)}{\mathrm{i} \hbar}\left(\Psi_{j^{\prime}}\left|\mu_{t}\right| \Psi_{j}\right),
\end{aligned}
$$

where $\left[\mu_{t}, \mathcal{H}_{0}\right]=\sum_{\ell=1}^{N}\left[\mu_{\ell}, p_{\ell, \mu}^{2} /(2 m)\right]=\mathrm{i} \hbar p_{t, \mu} / m$ and eq. (44) have been utilized. We also mention that $\Pi_{\mu}$ of eqs. (52) - (54) (or eq. (48)) is the $\mu$ component of $\left(\Psi_{1}\left|-\eta \lambda \frac{1}{\hbar}\left(\boldsymbol{r}_{t} \times \Delta \boldsymbol{p}_{\mathrm{e}}\right)\right| \Psi_{1}\right)$, where $\left(\Psi_{1}\left|p_{t, \mu}\right| \Psi_{1}\right)=0$ has been used in $V_{\mathrm{so} 2}^{(1)}$ of eq. (48) with eq. (31).

From now on, we will make no mention of eq. (49) or (50), which either contains only $a_{\xi}$ and $a_{\xi}^{\dagger}$ but no spin operators.

\subsection{Second-order perturbation energy}

Using $V_{\mathrm{p}}$ of eq. (23), $\left.\mid \Psi_{j}\right)\left|S_{z}\right\rangle$ of eq. (40), and $E_{j}$ of eq. (44), we obtain the second-order perturbation energy $V^{(2)}$, i.e.,

$$
V^{(2)}=\sum_{j(\neq 1)} \sum_{S_{z}{ }^{\prime \prime}} \frac{\left\langle S_{z}{ }^{\prime}\left|\left(\Psi_{1}\left|V_{\mathrm{p}}\right| \Psi_{j}\right)\right| S_{z}{ }^{\prime \prime}\right\rangle\left\langle S_{z}{ }^{\prime \prime}\left|\left(\Psi_{j}\left|V_{\mathrm{p}}\right| \Psi_{1}\right)\right| S_{z}\right\rangle}{E_{1}-E_{j}} .
$$

When terms higher than the first order of $\eta \Delta \xi_{\mathrm{n}}$ and $\eta \Delta p_{\mathrm{e}, \xi}$ with $\xi=x, y, z$ are neglected, $V^{(2)}$ is expressed as follows:

$$
\begin{aligned}
& V^{(2)}=V_{\mathrm{so} 0, \mathrm{so} 0}^{(2)}+V_{\mathrm{so} 0, \mathrm{so} 1}^{(2)}+V_{\mathrm{so} 0, \mathrm{so} 2}^{(2)}+V_{\mathrm{so} 0, \mathrm{k}}^{(2)}, \\
& V_{\mathrm{so} 0, \mathrm{so} 0}^{(2)}=f\left(V_{\mathrm{so} 0}, V_{\mathrm{so} 0}\right)=\sum_{\mu, \nu=x, y, z} \Lambda_{\mu, \nu} S_{\mu} S_{\nu}, \\
& V_{\mathrm{so} 0, \mathrm{so} 1}^{(2)}=f\left(V_{\mathrm{so} 0}, V_{\mathrm{so} 1}\right)+f\left(V_{\mathrm{so} 1}, V_{\mathrm{so} 0}\right) \\
& =\sum_{\mu, \nu, \xi=x, y, z} \Lambda_{\mu, \nu ; \xi} S_{\mu} S_{\nu}\left(a_{\xi}+a_{\xi}^{\dagger}\right), \\
& V_{\mathrm{so} 0, \mathrm{so} 2}^{(2)}=f\left(V_{\mathrm{so} 0}, V_{\mathrm{so} 2}\right)+f\left(V_{\mathrm{so} 2}, V_{\mathrm{so} 0}\right) \\
& =\sum_{\mu, \nu=x, y, z} \Gamma_{\mu, \nu} S_{\mu} S_{\nu} \\
& V_{\mathrm{so} 0, \mathrm{k}}^{(2)}=f\left(V_{\mathrm{so} 0}, V_{\mathrm{k}}^{\prime}\right)+f\left(V_{\mathrm{k}}^{\prime}, V_{\mathrm{so} 0}\right)=\sum_{\mu=x, y, z} k_{\mu} S_{\mu}
\end{aligned}
$$

where

$$
f(X, Y)=\sum_{j(\neq 1)} \sum_{S_{z}{ }^{\prime \prime}} \frac{\left\langle S_{z}{ }^{\prime}\left|\left(\Psi_{1}|X| \Psi_{j}\right)\right| S_{z}{ }^{\prime \prime}\right\rangle\left\langle S_{z}{ }^{\prime \prime}\left|\left(\Psi_{j}|Y| \Psi_{1}\right)\right| S_{z}\right\rangle}{E_{1}-E_{j}} .
$$

In the last results of eqs. (62) - (65), $\left\langle S_{z}^{\prime}\right|$ and $\left|S_{z}\right\rangle$ are omitted to use the operator representation. The quantities $\Lambda_{\mu, \nu}, \Lambda_{\mu, \nu ; \xi}, \Gamma_{\mu, \nu}$, and $k_{\mu}$ are given by

$$
\Lambda_{\mu, \nu}=\lambda^{2} \mathcal{L}_{\mu, \nu},
$$


J. Phys. Soc. Jpn.

Full PaPer

$$
\begin{aligned}
& \Lambda_{\mu, \nu ; \xi}=2 \eta \lambda^{2} \frac{\left\langle 3 \xi r^{-5}\right\rangle}{\left\langle r^{-3}\right\rangle} \sqrt{\frac{\hbar}{2 M \omega_{\xi}}} \mathcal{L}_{\mu, \nu}, \\
& \Gamma_{x, x}=\left(-\Lambda_{x ; z, y}^{+}+\Lambda_{x ; z, y}^{-}\right)\left(a_{y}+a_{y}^{\dagger}\right)+\left(\Lambda_{x ; y, z}^{+}-\Lambda_{x ; y, z}^{-}\right)\left(a_{z}+a_{z}^{\dagger}\right), \\
& \Gamma_{x, y}=\Lambda_{x ; z, x}^{+} a_{x}-\Lambda_{x ; z, x}^{-} a_{x}^{\dagger}+\Lambda_{y ; z, y}^{-} a_{y}-\Lambda_{y ; z, y}^{+} a_{y}^{\dagger}+\left(-\Lambda_{x ; x, z}^{+}-\Lambda_{y ; y, z}^{-}\right) a_{z}+\left(\Lambda_{y ; y, z}^{+}+\Lambda_{x ; x, z}^{-}\right) a_{z}^{\dagger}, \\
& \Gamma_{x, z}=-\Lambda_{x ; y, x}^{+} a_{x}+\Lambda_{x ; y, x}^{-} a_{x}^{\dagger}+\left(\Lambda_{x ; x, y}^{+}+\Lambda_{z ; z, y}^{-}\right) a_{y}+\left(-\Lambda_{z ; z, y}^{+}-\Lambda_{x ; x, y}^{-}\right) a_{y}^{\dagger}-\Lambda_{z ; y, z}^{-} a_{z}+\Lambda_{z ; y, z}^{+} a_{z}^{\dagger}, \\
& \Gamma_{y, x}=-\Lambda_{x ; z, x}^{-} a_{x}+\Lambda_{x ; z, x}^{+} a_{x}^{\dagger}-\Lambda_{y ; z, y}^{+} a_{y}+\Lambda_{y ; z, y}^{-} a_{y}^{\dagger}+\left(\Lambda_{y ; y, z}^{+}+\Lambda_{x ; x, z}^{-}\right) a_{z}+\left(-\Lambda_{x ; x, z}^{+}-\Lambda_{y ; y, z}^{-}\right) a_{z}^{\dagger}, \\
& \Gamma_{y, y}=\left(\Lambda_{y ; z, x}^{+}-\Lambda_{y ; z, x}^{-}\right)\left(a_{x}+a_{x}^{\dagger}\right)+\left(-\Lambda_{y ; x, z}^{+}+\Lambda_{y ; x, z}^{-}\right)\left(a_{z}+a_{z}^{\dagger}\right), \\
& \Gamma_{y, z}=\left(-\Lambda_{y ; y, x}^{+}-\Lambda_{z ; z, x}^{-}\right) a_{x}+\left(\Lambda_{z ; z, x}^{+}+\Lambda_{y ; y, x}^{-}\right) a_{x}^{\dagger}+\Lambda_{y ; x, y}^{+} a_{y}-\Lambda_{y ; x, y}^{-} a_{y}^{\dagger}+\Lambda_{z ; x, z}^{-} a_{z}-\Lambda_{z ; x, z}^{+} a_{z}^{\dagger}, \\
& \Gamma_{z, z}=\left(-\Lambda_{z ; y, x}^{+}+\Lambda_{z ; y, x}^{-}\right)\left(a_{x}+a_{x}^{\dagger}\right)+\left(\Lambda_{z ; x, y}^{+}-\Lambda_{z ; x, y}^{-}\right)\left(a_{y}+a_{y}^{\dagger}\right), \\
& k_{\mu}=\sum_{\xi=x, y, z} \Lambda_{\mu, \xi}^{k}\left(-a_{\xi}+a_{\xi}^{\dagger}\right), \\
& \Gamma_{z, x}=\Lambda_{x ; y, x}^{-} a_{x}-\Lambda_{x ; y, x}^{+} a_{x}^{\dagger}+\left(-\Lambda_{z ; z, y}^{+}-\Lambda_{x ; x, y}^{-}\right) a_{y}+\left(\Lambda_{x ; x, y}^{+}+\Lambda_{z ; z, y}^{-}\right) a_{y}^{\dagger}+\Lambda_{z ; y, z}^{+} a_{z}-\Lambda_{z ; y, z}^{-} a_{z}^{\dagger}, \\
& (745)
\end{aligned}
$$

respectively, where

$$
\begin{aligned}
& \mathcal{L}_{\mu, \nu}=\sum_{j(\neq 1)} \frac{\left(\Psi_{1}\left|L_{\mu}\right| \Psi_{j}\right)\left(\Psi_{j}\left|L_{\nu}\right| \Psi_{1}\right)}{E_{1}-E_{j}} \\
& \Lambda_{\mu, \xi}^{k}=-2 \eta \lambda \frac{m}{M} \frac{1}{\hbar} \sqrt{\frac{M \hbar \omega_{\xi}}{2}} \sum_{j(\neq 1)}\left(\Psi_{1}\left|L_{\mu}\right| \Psi_{j}\right)\left(\Psi_{j}\left|\xi_{t}\right| \Psi_{1}\right), \\
& \Lambda_{\mu ; \nu, \xi}^{ \pm}=\eta \lambda^{2} \frac{1}{\hbar} \sum_{j(\neq 1)} \frac{\left(\Psi_{1}\left|L_{\mu}\right| \Psi_{j}\right)\left(\Psi_{j}\left|F_{\nu, \xi}^{ \pm}\right| \Psi_{1}\right)}{E_{1}-E_{j}}, \\
& \left(\Psi_{j^{\prime}}\left|F_{\nu, \xi}^{ \pm}\right| \Psi_{j}\right)=\mathrm{i}\left[\frac{m}{M} \sqrt{\frac{M \hbar \omega_{\xi}}{2}} \pm \sqrt{\frac{\hbar}{2 M \omega_{\xi}}} \frac{m}{\hbar}\left(E_{j^{\prime}}-E_{j}\right)\right]\left(\Psi_{j^{\prime}}\left|\nu_{t}\right| \Psi_{j}\right), \\
& F_{\nu, \xi}^{ \pm}=\mathrm{i} \frac{m}{M} \sqrt{\frac{M \hbar \omega_{\xi}}{2}} \nu_{t} \pm \sqrt{\frac{\hbar}{2 M \omega_{\xi}}} p_{t, \nu} .
\end{aligned}
$$


J. Phys. Soc. Jpn.

Full PAPER

In the derivation of eq. (80), we have used eq. (59), $\left(\Psi_{j^{\prime}}|\boldsymbol{L}| \Psi_{j}\right)=-\left(\Psi_{j}|\boldsymbol{L}| \Psi_{j^{\prime}}\right),{ }^{42}$ and $\left(\Psi_{j^{\prime}}\left|\xi_{t}\right| \Psi_{j}\right)=\left(\Psi_{j}\left|\xi_{t}\right| \Psi_{j^{\prime}}\right)$. In addition, eq. (82) has been obtained by using eqs. (83) and (59). Here, we mention the relations of $\left(\Psi_{j^{\prime}}\left|F_{\nu, \xi}^{ \pm}\right| \Psi_{j}\right)^{*}=\left(\Psi_{j}\left|\left(F_{\nu, \xi}^{ \pm}\right)^{\dagger}\right| \Psi_{j^{\prime}}\right)=-\left(\Psi_{j}\left|F_{\nu, \xi}^{\mp}\right| \Psi_{j^{\prime}}\right)$ and $\left(\Psi_{j^{\prime}}\left|F_{\nu, \xi}^{ \pm}\right| \Psi_{j}\right)=\left(\Psi_{j}\left|F_{\nu, \xi}^{\mp}\right| \Psi_{j^{\prime}}\right)$, where $\left(\Psi_{j^{\prime}}\left|\nu_{t}\right| \Psi_{j}\right)=\left(\Psi_{j}\left|\nu_{t}\right| \Psi_{j^{\prime}}\right)$. We also show the relations of $\mathcal{L}_{\mu, \nu}=\mathcal{L}_{\nu, \mu},{ }^{43} \Lambda_{\mu, \nu}=\Lambda_{\nu, \mu}, \Lambda_{\mu, \nu ; \xi}=\Lambda_{\nu, \mu ; \xi}, \Lambda_{\mu ; \nu, \xi}^{ \pm}=-\Lambda_{\nu, \xi ; \mu}^{\mp}=\left(\Lambda_{\mu ; \nu, \xi}^{ \pm}\right)^{*},{ }^{44}$ and $\left(\Gamma_{\mu, \nu}\right)^{\dagger}=\Gamma_{\nu, \mu}$, where

$$
\Lambda_{\nu, \xi ; \mu}^{ \pm}=\eta \lambda^{2} \frac{1}{\hbar} \sum_{j(\neq 1)} \frac{\left(\Psi_{1}\left|F_{\nu, \xi}^{ \pm}\right| \Psi_{j}\right)\left(\Psi_{j}\left|L_{\mu}\right| \Psi_{1}\right)}{E_{1}-E_{j}} .
$$

Note that the two terms consisting of $V_{\mathrm{so} 0}$ and $V_{\mathrm{en}}^{\prime}+V_{\mathrm{c}}^{\prime}$ in eq. (60) cancel each other, i.e.,

$$
f\left(V_{\mathrm{so} 0}, V_{\mathrm{en}}^{\prime}+V_{\mathrm{c}}^{\prime}\right)+f\left(V_{\mathrm{en}}^{\prime}+V_{\mathrm{c}}^{\prime}, V_{\mathrm{so} 0}\right)=0,
$$

where $f(X, Y)$ is given by eq. (66). Here, we use $\left(\Psi_{j}|\boldsymbol{L}| \Psi_{1}\right)=-\left(\Psi_{1}|\boldsymbol{L}| \Psi_{j}\right)$ (see ref. 42$)$ and $\left(\Psi_{j}\left|V_{\mathrm{en}}^{\prime}+V_{\mathrm{c}}^{\prime}\right| \Psi_{1}\right)=\left(\Psi_{1}\left|V_{\mathrm{en}}^{\prime}+V_{\mathrm{c}}^{\prime}\right| \Psi_{j}\right)$. In particular, $f\left(V_{\mathrm{so} 0}, V_{\mathrm{c}}^{\prime}\right)+f\left(V_{\mathrm{c}}^{\prime}, V_{\mathrm{so} 0}\right)=0$ corresponds to the so-called Van Vleck cancellation. ${ }^{23,45}$

\subsection{Principal axis transformation}

To obtain expressions of $V_{\mathrm{so} 2}^{(1)}$ of eq. (48) and $V^{(2)}$ of eq. (61) in the principal axis coordinate system, we first introduce the rotational transformation matrix for the principal axis transformation. This transformation is based on the conventional theory for the anisotropy spin Hamiltonian. ${ }^{40,46}$ Here, $x, y$, and $z$ are regarded as the initial axes.

In accordance with refs. 40 and 46 , we focus on $\sum_{\mu, \nu=x, y, z} \mathcal{L}_{\mu, \nu} S_{\mu} S_{\nu}$ included in $V_{\mathrm{so} 0, \mathrm{so} 0}^{(2)}$ of eq. (62) and $V_{\mathrm{so} 0, \mathrm{so} 1}^{(2)}$ of eq. (63). The expression in the matrix representation is written as

$$
\begin{aligned}
\sum_{\mu, \nu=x, y, z} \mathcal{L}_{\mu, \nu} S_{\mu} S_{\nu} & =\left(S_{x}, S_{y}, S_{z}\right)\left(\begin{array}{ccc}
\mathcal{L}_{x, x} & \mathcal{L}_{x, y} & \mathcal{L}_{x, z} \\
\mathcal{L}_{y, x} & \mathcal{L}_{y, y} & \mathcal{L}_{y, z} \\
\mathcal{L}_{z, x} & \mathcal{L}_{z, y} & \mathcal{L}_{z, z}
\end{array}\right)\left(\begin{array}{c}
S_{x} \\
S_{y} \\
S_{z}
\end{array}\right) \\
& \equiv \boldsymbol{S} \mathcal{L} \boldsymbol{S}^{T},
\end{aligned}
$$

with

$$
\begin{aligned}
\mathcal{L} & =\left(\begin{array}{ccc}
\mathcal{L}_{x, x} & \mathcal{L}_{x, y} & \mathcal{L}_{x, z} \\
\mathcal{L}_{y, x} & \mathcal{L}_{y, y} & \mathcal{L}_{y, z} \\
\mathcal{L}_{z, x} & \mathcal{L}_{z, y} & \mathcal{L}_{z, z}
\end{array}\right) \\
& =\mathcal{L}^{(0)}+\mathcal{L}^{\prime}, \\
\mathcal{L}^{(0)} & =\left(\begin{array}{ccc}
\mathcal{L}_{x, x}^{(0)} & \mathcal{L}_{x, y}^{(0)} & \mathcal{L}_{x, z}^{(0)} \\
\mathcal{L}_{y, x}^{(0)} & \mathcal{L}_{y, y}^{(0)} & \mathcal{L}_{y, z}^{(0)} \\
\mathcal{L}_{z, x}^{(0)} & \mathcal{L}_{z, y}^{(0)} & \mathcal{L}_{z, z}^{(0)}
\end{array}\right),
\end{aligned}
$$


J. Phys. Soc. Jpn.

Full PaPer

$$
\begin{aligned}
\mathcal{L}^{\prime} & =\left(\begin{array}{ccc}
\mathcal{L}_{x, x}^{\prime} & \mathcal{L}_{x, y}^{\prime} & \mathcal{L}_{x, z}^{\prime} \\
\mathcal{L}_{y, x}^{\prime} & \mathcal{L}_{y, y}^{\prime} & \mathcal{L}_{y, z}^{\prime} \\
\mathcal{L}_{z, x}^{\prime} & \mathcal{L}_{z, y}^{\prime} & \mathcal{L}_{z, z}^{\prime}
\end{array}\right) \\
\mathcal{L}_{\mu, \nu}^{(0)} & =\sum_{j(\neq 1)} \frac{\left\langle\Phi_{1}\left|L_{\mu}\right| \Phi_{j}\right\rangle\left\langle\Phi_{j}\left|L_{\nu}\right| \Phi_{1}\right\rangle}{e_{1}-e_{j}}, \\
\mathcal{L}_{\mu, \nu}^{\prime} & =\mathcal{L}_{\mu, \nu}-\mathcal{L}_{\mu, \nu}^{(0)}
\end{aligned}
$$

where $\boldsymbol{S}^{T}$ is the transposed vector of $\boldsymbol{S}=\left(S_{x}, S_{y}, S_{z}\right)$. Here, $\mathcal{L}_{\mu, \nu}^{(0)}$ is described by $\Phi_{j}$ consisting of only $d_{\zeta}(\ell)$, whereas $\mathcal{L}_{\mu, \nu}^{\prime}$ is related to $\overline{d_{m}}(\ell), \overline{p_{n}}(\ell)$, and $d_{\zeta}(\ell)$. We also have $\mathcal{L}_{\mu, \nu}^{(0)}=\mathcal{L}_{\nu, \mu}^{(0)}$ and $\mathcal{L}_{\mu, \nu}^{\prime}=\mathcal{L}_{\nu, \mu}^{\prime}{ }^{43}$

The principal axis of the crystal, named $X, Y$, and $Z$, are considered to be the coordinate system that diagonalizes $\mathcal{L}^{(0)}{ }^{40,46}$ We then introduce the matrices $\boldsymbol{U}$ and $\boldsymbol{U}^{T}$ that diagonalize $\mathcal{L}^{(0)}$. Here, $\boldsymbol{U}$ is the rotational transformation matrix, while the transposed matrix of $\boldsymbol{U}, \boldsymbol{U}^{T}$, satisfies $\boldsymbol{U}^{T}=\boldsymbol{U}^{-1}$. We thus have

$$
\begin{gathered}
\boldsymbol{U} \mathcal{L}^{(0)} \boldsymbol{U}^{T}=\left(\begin{array}{ccc}
\mathcal{L}_{X, X}^{(0)} & 0 & 0 \\
0 & \mathcal{L}_{Y, Y}^{(0)} & 0 \\
0 & 0 & \mathcal{L}_{Z, Z}^{(0)}
\end{array}\right), \\
\boldsymbol{U} \mathcal{L}^{\prime} \boldsymbol{U}^{T}=\left(\begin{array}{ccc}
\mathcal{L}_{X, X}^{\prime} & \mathcal{L}_{X, Y}^{\prime} & \mathcal{L}_{X, Z}^{\prime} \\
\mathcal{L}_{Y, X}^{\prime} & \mathcal{L}_{Y, Y}^{\prime} & \mathcal{L}_{Y, Z}^{\prime} \\
\mathcal{L}_{Z, X}^{\prime} & \mathcal{L}_{Z, Y}^{\prime} & \mathcal{L}_{Z, Z}^{\prime}
\end{array}\right),
\end{gathered}
$$

where

$$
\begin{aligned}
& \boldsymbol{U}=\left(\begin{array}{ccc}
\cos \theta_{X, x} & \cos \theta_{X, y} & \cos \theta_{X, z} \\
\cos \theta_{Y, x} & \cos \theta_{Y, y} & \cos \theta_{Y, z} \\
\cos \theta_{Z, x} & \cos \theta_{Z, y} & \cos \theta_{Z, z}
\end{array}\right), \\
& \boldsymbol{U}^{T}=\left(\begin{array}{lll}
\cos \theta_{X, x} & \cos \theta_{Y, x} & \cos \theta_{Z, x} \\
\cos \theta_{X, y} & \cos \theta_{Y, y} & \cos \theta_{Z, y} \\
\cos \theta_{X, z} & \cos \theta_{Y, z} & \cos \theta_{Z, z}
\end{array}\right), \\
& \mathcal{L}_{I, I}^{(0)}=\sum_{\mu, \nu=x, y, z} \cos \theta_{I, \mu} \cos \theta_{I, \nu} \mathcal{L}_{\mu, \nu}^{(0)}, \\
& \mathcal{L}_{I, J}^{\prime}=\sum_{\mu, \nu=x, y, z} \cos \theta_{I, \mu} \cos \theta_{J, \nu} \mathcal{L}_{\mu, \nu}^{\prime},
\end{aligned}
$$

with $I, J=X, Y, Z$. The angle $\theta_{I, \mu}$ is the relative angle between the $I$ and $\mu$ axes, with $I=X$, $Y, Z$ and $\mu=x, y, z$. By utilizing $\mathcal{L}_{\mu, \nu}^{\prime}=\mathcal{L}_{\nu, \mu}^{\prime},{ }^{43} \mathcal{L}_{I, J}^{\prime}=\sum_{\mu, \nu=x, y, z} \cos \theta_{J, \nu} \cos \theta_{I, \mu} \mathcal{L}_{\nu, \mu}^{\prime}=\mathcal{L}_{J, I}^{\prime}$ is obtained. 
J. Phys. Soc. Jpn.

Full PAPER

Using $\boldsymbol{U}$ and $\boldsymbol{U}^{T}$, we write eq. (86) as

$$
\begin{aligned}
\sum_{\mu, \nu=x, y, z} \mathcal{L}_{\mu, \nu} S_{\mu} S_{\nu}= & \left(S_{x}, S_{y}, S_{z}\right) \boldsymbol{U}^{T} \boldsymbol{U}\left(\begin{array}{ccc}
\mathcal{L}_{x, x} & \mathcal{L}_{x, y} & \mathcal{L}_{x, z} \\
\mathcal{L}_{y, x} & \mathcal{L}_{y, y} & \mathcal{L}_{y, z} \\
\mathcal{L}_{z, x} & \mathcal{L}_{z, y} & \mathcal{L}_{z, z}
\end{array}\right) \boldsymbol{U}^{T} \boldsymbol{U}\left(\begin{array}{c}
S_{x} \\
S_{y} \\
S_{z}
\end{array}\right) \\
= & \left(S_{X}, S_{Y}, S_{Z}\right)\left(\begin{array}{ccc}
\mathcal{L}_{X, X}^{(0)} & 0 & 0 \\
0 & \mathcal{L}_{Y, Y}^{(0)} & 0 \\
0 & 0 & \mathcal{L}_{Z, Z}^{(0)}
\end{array}\right)\left(\begin{array}{c}
S_{X} \\
S_{Y} \\
S_{Z}
\end{array}\right) \\
& +\left(S_{X}, S_{Y}, S_{Z}\right)\left(\begin{array}{ccc}
\mathcal{L}_{X, X}^{\prime} & \mathcal{L}_{X, Y}^{\prime} & \mathcal{L}_{X, Z}^{\prime} \\
\mathcal{L}_{Y, X}^{\prime} & \mathcal{L}_{Y, Y}^{\prime} & \mathcal{L}_{Y, Z}^{\prime} \\
\mathcal{L}_{Z, X}^{\prime} & \mathcal{L}_{Z, Y}^{\prime} & \mathcal{L}_{Z, Z}^{\prime}
\end{array}\right)\left(\begin{array}{c}
S_{X} \\
S_{Y} \\
S_{Z}
\end{array}\right) \\
= & \sum_{I=X, Y, Z} \mathcal{L}_{I, I}^{(0)} S_{I}^{2}+\sum_{I, J=X, Y, Z} \mathcal{L}_{I, J}^{\prime} S_{I} S_{J},
\end{aligned}
$$

where the relation between $\left(S_{X}, S_{Y}, S_{Z}\right)$ in the principal axis $(X, Y, Z)$ and $\left(S_{x}, S_{y}, S_{z}\right)$ in the initial axes $(x, y, z)$ has been defined by

$$
\left(\begin{array}{c}
S_{X} \\
S_{Y} \\
S_{Z}
\end{array}\right)=\boldsymbol{U}\left(\begin{array}{c}
S_{x} \\
S_{y} \\
S_{z}
\end{array}\right)
$$

\subsection{Expressions of spin-atomic vibration interaction and spin-flip Hamiltonian}

Using the above-mentioned $\boldsymbol{U}$ and $\boldsymbol{U}^{T}$, we can obtain the expressions of $V_{\mathrm{so} 2}^{(1)}$ of eq. (48) and $V^{(2)}\left(=V_{\mathrm{so} 0, \mathrm{so} 0}^{(2)}+V_{\mathrm{so} 0, \mathrm{so} 1}^{(2)}+V_{\mathrm{so} 0, \mathrm{so} 2}^{(2)}+V_{\mathrm{so} 0, \mathrm{k}}^{(2)}\right)$ of eq. (61) in the principal axis coordinate system. Each term is named any one of the spin-atomic vibration interaction, spin-flip Hamiltonian, or anisotropy spin Hamiltonian. In addition, we consider the feature of the respective terms. Their coefficients and operators are listed in Table I.

3.4.1 $v_{\mathrm{so} 0, \mathrm{so} 0,1}^{(2)}$ and $v_{\mathrm{so} 0, \mathrm{so} 0,2}^{(2)}$

Using eq. (98), we first rewrite $V_{\mathrm{so} 0, \mathrm{so} 0}^{(2)}$ of eq. (62) as

$$
\begin{aligned}
& V_{\mathrm{so} 0, \mathrm{so} 0}^{(2)}=v_{\mathrm{so} 0, \mathrm{so} 0,1}^{(2)}+v_{\mathrm{so} 0, \mathrm{so} 0,2}^{(2)}, \\
& v_{\mathrm{so} 0, \mathrm{so} 0,1}^{(2)}=D S_{Z}^{2}+E\left(S_{X}^{2}-S_{Y}^{2}\right)+F S(S+1), \\
& v_{\mathrm{so} 0, \mathrm{so} 0,2}^{(2)}=\sum_{I, J=X, Y, Z} \Lambda_{I, J}^{\mathrm{SF}} S_{I} S_{J},
\end{aligned}
$$

with

$$
\begin{aligned}
& D=\lambda^{2}\left[\mathcal{L}_{Z, Z}^{(0)}-\left(\mathcal{L}_{X, X}^{(0)}+\mathcal{L}_{Y, Y}^{(0)}\right) / 2\right] \\
& E=\lambda^{2}\left(\mathcal{L}_{X, X}^{(0)}-\mathcal{L}_{Y, Y}^{(0)}\right) / 2, \\
& F=\lambda^{2}\left(\mathcal{L}_{X, X}^{(0)}+\mathcal{L}_{Y, Y}^{(0)}\right) / 2,
\end{aligned}
$$


J. Phys. Soc. Jpn.

Full PaPer

$$
\Lambda_{I, J}^{\mathrm{SF}}=\lambda^{2} \mathcal{L}_{I, J}^{\prime}
$$

where we have $\Lambda_{I, J}^{\mathrm{SF}}=\Lambda_{J, I}^{\mathrm{SF}}$ using $\mathcal{L}_{I, J}^{\prime}=\mathcal{L}_{J, I}^{\prime}$ (see $\S 3.3$ ). The Hamiltonian $v_{\mathrm{so} 0, \mathrm{so} 0,1}^{(2)}$ is the socalled anisotropy spin Hamiltonian, ${ }^{40}$ where $D$ is an anisotropy constant. The Hamiltonian $v_{\mathrm{so0}, \mathrm{so} 0,2}^{(2)}$, which contains the spin-flip operators such as $S_{X} S_{Z}$ and $S_{Y} S_{Z}$ but no atomic vibration operators, is named the spin-flip Hamiltonian.

From now on, $F S(S+1)$ in eq. (101) will be neglected because of a constant term.

3.4.2 $v_{\mathrm{so} 0, \mathrm{so} 1,1}^{(2)}$ and $v_{\mathrm{so} 0, \mathrm{so} 1,2}^{(2)}$

Using eq. (98), we express $V_{\mathrm{so} 0, \text { so } 1}^{(2)}$ of eq. (63) as

$$
\begin{aligned}
& V_{\mathrm{so} 0, \mathrm{so} 1}^{(2)}=v_{\mathrm{so} 0, \mathrm{so} 1,1}^{(2)}+v_{\mathrm{so} 0, \mathrm{so} 1,2}^{(2)}, \\
& v_{\mathrm{so} 0, \mathrm{so} 1,1}^{(2)}=\left[D S_{Z}^{2}+E\left(S_{X}^{2}-S_{Y}^{2}\right)+F S(S+1)\right] \sum_{\xi=x, y, z} G_{\xi}\left(a_{\xi}+a_{\xi}^{\dagger}\right), \\
& v_{\mathrm{so} 0, \mathrm{so} 1,2}^{(2)}=\sum_{\xi=x, y, z} \sum_{I, J=X, Y, X} \Lambda_{I, J ; \xi}^{\prime} S_{I} S_{J}\left(a_{\xi}+a_{\xi}^{\dagger}\right),
\end{aligned}
$$

with

$$
\begin{aligned}
& G_{\xi}=2 \eta \frac{\left\langle 3 \xi r^{-5}\right\rangle}{\left\langle r^{-3}\right\rangle} \sqrt{\frac{\hbar}{2 M \omega_{\xi}}}, \\
& \Lambda_{I, J ; \xi}^{\prime}=2 \eta \lambda^{2} \frac{\left\langle 3 \xi r^{-5}\right\rangle}{\left\langle r^{-3}\right\rangle} \sqrt{\frac{\hbar}{2 M \omega_{\xi}}} \mathcal{L}_{I, J}^{\prime},
\end{aligned}
$$

where we have $\Lambda_{I, J ; \xi}^{\prime}=\Lambda_{J, I ; \xi}^{\prime}$ using $\mathcal{L}_{I, J}^{\prime}=\mathcal{L}_{J, I}^{\prime}$ (see $\S 3.3$ ). The interactions $v_{\mathrm{so0}, \mathrm{so} 1,1}^{(2)}$ and $v_{\mathrm{So} 0, \mathrm{so} 1,2}^{(2)}$, which contain the spin and vibration operators, are named the spin-atomic vibration interactions.

\subsection{3 $V_{\mathrm{so} 0, \mathrm{so} 2}^{(2)}$}

Using $\boldsymbol{U}$ and $\boldsymbol{U}^{T}$, we rewrite $V_{\mathrm{so0}, \mathrm{so} 2}^{(2)}$ of eq. (64) as

$$
\begin{aligned}
& V_{\mathrm{so} 0, \mathrm{so} 2}^{(2)}=\sum_{\mu, \nu=x, y, z} \Gamma_{\mu, \nu} S_{\mu} S_{\nu} \\
& =\left(S_{x}, S_{y}, S_{z}\right) \boldsymbol{U}^{T} \boldsymbol{U}\left(\begin{array}{ccc}
\Gamma_{x, x} & \Gamma_{x, y} & \Gamma_{x, z} \\
\Gamma_{y, x} & \Gamma_{y, y} & \Gamma_{y, z} \\
\Gamma_{z, x} & \Gamma_{z, y} & \Gamma_{z, z}
\end{array}\right) \boldsymbol{U}^{T} \boldsymbol{U}\left(\begin{array}{c}
S_{x} \\
S_{y} \\
S_{z}
\end{array}\right) \\
& =\left(S_{X}, S_{Y}, S_{Z}\right)\left(\begin{array}{ccc}
\Gamma_{X, X} & \Gamma_{X, Y} & \Gamma_{X, Z} \\
\Gamma_{Y, X} & \Gamma_{Y, Y} & \Gamma_{Y, Z} \\
\Gamma_{Z, X} & \Gamma_{Z, Y} & \Gamma_{Z, Z}
\end{array}\right)\left(\begin{array}{c}
S_{X} \\
S_{Y} \\
S_{Z}
\end{array}\right) \\
& =\sum_{I, J=X, Y, Z} \Gamma_{I, J} S_{I} S_{J},
\end{aligned}
$$


where

$$
\Gamma_{I, J}=\sum_{\mu, \nu=x, y, z} \cos \theta_{I, \mu} \cos \theta_{J, \nu} \Gamma_{\mu, \nu}
$$

with $\Gamma_{\mu, \nu}$ being eqs. (69) - (77). We here mention a relation of $\left(\Gamma_{I, J}\right)^{\dagger}=$ $\sum_{\mu, \nu=x, y, z} \cos \theta_{J, \nu} \cos \theta_{I, \mu} \Gamma_{\nu, \mu}=\Gamma_{J, I}$ using $\left(\Gamma_{\mu, \nu}\right)^{\dagger}=\Gamma_{\nu, \mu}$ (see $\left.\S 3.2\right)$. The interaction $V_{\mathrm{so} 0, \mathrm{so} 2}^{(2)}$, which contains the spin and vibration operators, is the spin-atomic vibration interaction.

\section{$3.4 .4 \quad V_{\mathrm{soO}, \mathrm{k}}^{(2)}$}

We write $V_{\mathrm{so0}, \mathrm{k}}^{(2)}$ of eq. (65) by

$$
\begin{aligned}
V_{\mathrm{so} 0, \mathrm{k}}^{(2)} & =\sum_{\mu=x, y, z} k_{\mu} S_{\mu} \\
& =\left(k_{x}, k_{y}, k_{z}\right) \boldsymbol{U}^{T} \boldsymbol{U}\left(\begin{array}{c}
S_{x} \\
S_{y} \\
S_{z}
\end{array}\right) \\
& =\sum_{I=X, Y, Z} k_{I} S_{I},
\end{aligned}
$$

with

$$
k_{I}=\sum_{\mu=x, y, z} k_{\mu} \cos \theta_{I, \mu},
$$

where $k_{\mu}$ is eq. (78). The interaction $V_{\mathrm{so} 0, \mathrm{k}}^{(2)}$, which contains the spin and vibration operators, is the spin-atomic vibration interaction.

\subsection{5 $V_{\mathrm{so} 2}^{(1)}$}

We express $V_{\mathrm{so} 2}^{(1)}$ of eq. (48) by

$$
\begin{aligned}
V_{\mathrm{so} 2}^{(1)} & =\sum_{\mu=x, y, z} \Pi_{\mu} S_{\mu} \\
& =\left(\Pi_{x}, \Pi_{y}, \Pi_{z}\right) \boldsymbol{U}^{T} \boldsymbol{U}\left(\begin{array}{c}
S_{x} \\
S_{y} \\
S_{z}
\end{array}\right) \\
& =\Pi_{X} S_{X}+\Pi_{Y} S_{Y}+\Pi_{Z} S_{Z} \\
& =\sum_{I=X, Y, Z} \Pi_{I} S_{I},
\end{aligned}
$$

with

$$
\Pi_{I}=\sum_{\mu=x, y, z} \Pi_{\mu} \cos \theta_{I, \mu}
$$

where $\Pi_{x}, \Pi_{y}$, and $\Pi_{z}$ are eqs. (52) - (54), respectively. The interaction $V_{\text {so2 }}^{(1)}$, which contains the spin and vibration operators, is the spin-atomic vibration interaction. 


\subsubsection{Considerations}

On the basis of $§ 3.4 .1-\S 3.4 .5$, we finally express the perturbation energy, $V_{\mathrm{so} 2}^{(1)}+V^{(2)}$, as

$$
\begin{aligned}
V & =V_{\mathrm{so} 2}^{(1)}+v_{\mathrm{so} 0, \mathrm{so} 0,1}^{(2)}+v_{\mathrm{so} 0, \mathrm{so} 0,2}^{(2)}+v_{\mathrm{so} 0, \mathrm{so} 1,1}^{(2)}+v_{\mathrm{so} 0, \mathrm{so} 1,2}^{(2)}+V_{\mathrm{so} 0, \mathrm{so} 2}^{(2)}+V_{\mathrm{so} 0, \mathrm{k}}^{(2)} \\
& \equiv V_{\mathrm{A}}+V_{\mathrm{SA}}+V_{\mathrm{SF}}
\end{aligned}
$$

where $V_{\mathrm{A}}, V_{\mathrm{SA}}$, and $V_{\mathrm{SF}}$ are the anisotropy spin Hamiltonian, spin-atomic vibration interaction, and spin-flip Hamiltonian, respectively. These terms are written by the following simplified expressions:

$$
\begin{aligned}
V_{\mathrm{A}} \equiv & v_{\mathrm{So} 0, \mathrm{so} 0,1}^{(2)}=D S_{Z}^{2}+E\left(S_{X}^{2}-S_{Y}^{2}\right) \\
V_{\mathrm{SA}} \equiv & V_{\mathrm{so} 2}^{(1)}+v_{\mathrm{so} 0, \mathrm{so} 1,1}^{(2)}+v_{\mathrm{so} 0, \mathrm{so} 1,2}^{(2)}+V_{\mathrm{so} 0, \mathrm{so} 2}^{(2)}+V_{\mathrm{so} 0, \mathrm{k}}^{(2)} \\
= & \sum_{I=X, Y, Z} \sum_{\xi=x, y, z} c_{I, \xi} S_{I}\left(-a_{\xi}+a_{\xi}^{\dagger}\right) \\
& +\sum_{I, J=X, Y, Z} \sum_{\xi=x, y, z} S_{I} S_{J}\left(c_{1, I, J, \xi} a_{\xi}+c_{2, I, J, \xi} a_{\xi}^{\dagger}\right) \\
& +\left[D S_{Z}^{2}+E\left(S_{X}^{2}-S_{Y}^{2}\right)+F S(S+1)\right] \sum_{\xi=x, y, z} G_{\xi}\left(a_{\xi}+a_{\xi}^{\dagger}\right), \\
V_{\mathrm{SF}} \equiv & v_{\mathrm{so} 0, \mathrm{so} 0,2}^{(2)}=\sum_{I, J=X, Y, Z} \Lambda_{I, J}^{\mathrm{SF}} S_{I} S_{J},
\end{aligned}
$$

where $c_{I, \xi}$ is the coefficient of the $S_{I}\left(-a_{\xi}+a_{\xi}^{\dagger}\right)$ term, and $c_{1, I, J, \xi}\left(c_{2, I, J, \xi}\right)$ is that of the $S_{I} S_{J} a_{\xi}$ term (that of the $S_{I} S_{J} a_{\xi}^{\dagger}$ term). Here, $c_{I, \xi}$ consists of $\Lambda_{\mu, \xi}^{(1)}$ of eq. (55) and $\Lambda_{\mu, \xi}^{k}$ of eq. (80), while $c_{n, I, J, \xi}(n=1,2)$ is composed of $\Lambda_{I, J ; \xi}^{\prime}$ of eq. (111) and $\Lambda_{\mu ; \nu, \xi}^{ \pm}$of eq. (81). In other words, $V_{\mathrm{so} 2}^{(1)}$ and $V_{\mathrm{so} 0, \mathrm{k}}^{(2)}$ are terms with $S_{I}\left(-a_{\xi}+a_{\xi}^{\dagger}\right)$, while $v_{\mathrm{so} 0, \mathrm{so} 1,2}^{(2)}$ and $V_{\mathrm{so} 0, \mathrm{so} 2}^{(2)}$ are those with $S_{I} S_{J} a_{\xi}$ and $S_{I} S_{J} a_{\xi}^{\dagger}$.

We consider the features of $V_{\mathrm{So} 2}^{(1)}, V_{\mathrm{so} 0, \mathrm{k}}^{(2)}, v_{\mathrm{so} 0, \mathrm{so} 1,1}^{(2)}, v_{\mathrm{so} 0, \mathrm{so} 1,2}^{(2)}, V_{\mathrm{so} 0, \mathrm{so} 2}^{(2)}$ of $V_{\mathrm{SA}}$, and $v_{\mathrm{so} 0, \mathrm{so} 0,2}^{(2)}$ of $V_{\mathrm{SF}}$. We first find that $V_{\mathrm{So} 2}^{(1)}$ and $V_{\mathrm{so} 0, \mathrm{k}}^{(2)}$ are proportional to $\lambda$, while $v_{\mathrm{so} 0, \mathrm{so} 1,1}^{(2)}, v_{\mathrm{so} 0, \mathrm{so} 1,2}^{(2)}$, $V_{\mathrm{so} 0, \mathrm{so} 2}^{(2)}$, and $v_{\mathrm{So} 0, \mathrm{so} 0,2}^{(2)}$ are proportional to $\lambda^{2}$ (see Table I). Second, the presence or absence of the respective terms is shown by examining the respective coefficients for each set of $\eta, c_{p}$, $c_{d}$, where $c_{d}$ and $c_{p}$ are defined as $c_{d}=c_{d_{m}}^{(\zeta)}$ and $c_{p}=c_{p_{n}}^{(\zeta)}$, respectively (see Table I). Here, $\omega_{\xi}$ is put to be $\omega_{\xi}=2 \pi f_{\xi}$ for $\xi=x, y, z$, where $f_{\xi}$ is the vibration frequency in the $\xi$ direction. In addition, $e_{1}-e_{j}=E_{1}-E_{j}$ is roughly set by taking into account $\left|\Delta e_{1}-\Delta e_{j}\right| \ll\left|e_{1}-e_{j}\right|$ for $j \neq 1$ (see eq. (45)). The details are described as follows:

- Spin-atomic vibration interactions $V_{\mathrm{SA}}$

$$
S_{I}\left(-a_{\xi}+a_{\xi}^{\dagger}\right) \text { term }
$$

$-V_{\mathrm{so} 2}^{(1)}$ with $\Lambda_{\mu, \xi}^{(1)} \propto \eta \sqrt{f_{\xi}} \quad$ (see eq. (55))

The interaction $V_{\mathrm{so} 2}^{(1)}$ appears for $\eta \neq 0$ and $c_{p} \neq 0$, because each term contains 
$\eta\left(\Psi_{j^{\prime}}\left|\mu_{t}\right| \Psi_{j}\right)$, with $\mu=x, y, z$. Here, $\left(\Psi_{j^{\prime}}\left|\mu_{t}\right| \Psi_{j}\right)$ is the same formula as a matrix element of the so-called electric dipole transition; it is nonzero for $c_{p} \neq 0$. In addition, $V_{\mathrm{So} 2}^{(1)}$ is proportional to $\sqrt{f_{\xi}}$, reflecting that $V_{\mathrm{so} 2}^{(1)}$ contains $\Delta p_{\mathrm{n}, \xi}$ of eq. (58), with $\xi=x, y, z$.

$-V_{\mathrm{so} 0, \mathrm{k}}^{(2)}$ with $\Lambda_{\mu, \xi}^{k} \propto \eta \sqrt{f_{\xi}}$ (see eq. (80))

The interaction $V_{\mathrm{so}, \mathrm{k}}^{(2)}$, in which each term contains $\eta\left(\Psi_{j}\left|\xi_{t}\right| \Psi_{1}\right)$ with $\xi=x, y, z$, is present for $\eta \neq 0$ and $c_{p} \neq 0$. In addition, $V_{\mathrm{so} 0, \mathrm{k}}^{(2)}$ is proportional to $\sqrt{f_{\xi}}$ because $V_{\mathrm{so} 0, \mathrm{k}}^{(2)}$ (or $\left.V_{\mathrm{k}}^{\prime}\right)$ contains $\Delta p_{\mathrm{n}, \xi}$.

$S_{I} S_{J} a_{\xi}$ and $S_{I} S_{J} a_{\xi}^{\dagger}$ terms

$-V_{\mathrm{so} 0, \mathrm{so} 2}^{(2)}$ with $\Lambda_{\mu ; \nu, \xi}^{ \pm} \propto \eta \quad$ (see eq. (81))

The interaction $V_{\mathrm{so} 0, \mathrm{so} 2}^{(2)}$ appears for $\eta \neq 0$ and $c_{p} \neq 0$, because each term has $\eta\left(\Psi_{j^{\prime}}\left|\nu_{t}\right| \Psi_{1}\right)$, with $\nu=x, y, z$. In addition, $V_{\mathrm{so} 0, \mathrm{so} 2}^{(2)}$ contains both terms with $\sqrt{f_{\xi}}$ and those with $1 / \sqrt{f_{\xi}}$, reflecting that $V_{\mathrm{so} 0, \mathrm{so} 2}^{(2)}\left(\right.$ or $\left.V_{\mathrm{so} 2}\right)$ has $\Delta p_{\mathrm{n}, \xi}$ and $\Delta \xi_{\mathrm{n}}$ of eq. (56), with $\xi=x, y, z$. $-v_{\mathrm{so} 0, \mathrm{so} 1,1}^{(2)}$ with $G_{\xi} \propto \eta\left\langle 3 \xi r^{-5}\right\rangle / \sqrt{f_{\xi}} \quad$ (see eq. (110))

The interaction $v_{\mathrm{so} 0, \mathrm{so} 1,1}^{(2)}$ exists for $\eta \neq 0$ and $c_{p} \neq 0$, because it is proportional to $\eta\left\langle 3 \xi r^{-5}\right\rangle$, with $\xi=x, y, z$. Here, $\left\langle 3 \xi r^{-5}\right\rangle$ is nonzero for $c_{p} \neq 0$. It is noted that the operator is proportional to $\xi$ as with the matrix element of the electric dipole transition. In addition, $v_{\mathrm{so} 0, \mathrm{so} 1,1}^{(2)}$ is proportional to $1 / \sqrt{f_{\xi}}$ because $v_{\mathrm{so} 0, \mathrm{so} 1,1}^{(2)}\left(\right.$ or $\left.V_{\mathrm{so} 1}\right)$ contains $\Delta \xi_{\mathrm{n}}$.

$-v_{\mathrm{so} 0, \mathrm{so} 1,2}^{(2)}$ with $\Lambda_{I, J ; \xi}^{\prime} \propto \eta\left\langle 3 \xi r^{-5}\right\rangle \mathcal{L}_{I, J}^{\prime} / \sqrt{f_{\xi}} \quad$ (see eq. (111))

The interaction $v_{\mathrm{so}, \mathrm{so} 1,2}^{(2)}$ is present for $\eta \neq 0, c_{p} \neq 0$, and $c_{d} \neq 0$, because it is proportional to $\eta\left\langle 3 \xi r^{-5}\right\rangle \mathcal{L}_{I, J}^{\prime}$ with $\xi=x, y, z$. Here, $\mathcal{L}_{I, J}^{\prime}$ is nonzero for $c_{d} \neq 0$. In addition, $v_{\mathrm{so} 0, \mathrm{so} 1,2}^{(2)}$ is proportional to $1 / \sqrt{f_{\xi}}$, reflecting that $v_{\mathrm{so} 0, \mathrm{so} 1,2}^{(2)}\left(\right.$ or $\left.V_{\mathrm{so} 1}\right)$ has $\Delta \xi_{\mathrm{n}}$.

- Spin-flip Hamiltonian $V_{\mathrm{SF}}$

$-v_{\mathrm{so} 0, \mathrm{so} 0,2}^{(2)}$ with $\Lambda_{I, J}^{\mathrm{SF}} \propto \mathcal{L}_{I, J}^{\prime} \quad$ (see eq. (106))

The Hamiltonian $v_{\mathrm{so}, \mathrm{so} 0,2}^{(2)}$, which is proportional to $\mathcal{L}_{I, J}^{\prime}$, exists for $c_{d} \neq 0$. Note that $v_{\mathrm{so} 0, \mathrm{so} 0,2}^{(2)}$ is independent of the atomic vibration, and it does not contain $\eta$ and $f_{\xi}$.

\section{Application to Fe Ion}

As an application, we consider the spin-atomic vibration interaction and the spin-flip Hamiltonian of the $\mathrm{Fe}$ ion $\left(\mathrm{Fe}^{2+}\right)$ in a crystal field of tetragonal symmetry, where $\mathrm{Fe}^{2+}$ has six $3 \mathrm{~d}$ electrons (i.e., $3 \mathrm{~d}^{6}$ ). We first investigate the $f$ dependence of their coefficients. Second, their coefficients are evaluated by assigning appropriate values to $\lambda$.

\subsection{Orbital and spin states}

As to the Fe ion $\left(3 \mathrm{~d}^{6}\right)$, the ground state consists of five up-spin electrons and one downspin electron, according to Hund's first rule. The spin $S$ is therefore considered to be $S=2$. In addition, since the up-spin shell is filled, $\Psi_{j}$ of eq. (41) is given by the orbital wave function 
J. Phys. Soc. Jpn.

Full PAPER

$$
\begin{aligned}
& \Delta_{l}=E_{5} \quad\left(E_{3 z^{2}-r^{2}}\right) \\
& \Delta_{m}=E_{4} \quad\left(E_{x^{2}-y^{2}}\right) \\
& \Delta_{s}=E_{2}=E_{3} \quad\left(E_{x z}=E_{y z}\right) \\
& 0=E_{1} \quad\left(E_{x y}\right)
\end{aligned}
$$

Fig. 2. Energy level $E_{j}$ of the down-spin shell of the 3 d orbitals of the $\mathrm{Fe}^{2+}\left(3 \mathrm{~d}^{6}\right)$ in the crystal field of tetragonal symmetry. We use the notation of $E_{1}=E_{x y}, E_{2}=E_{x z}, E_{3}=E_{y z}, E_{4}=E_{x^{2}-y^{2}}$, and $E_{5}=E_{3 z^{2}-r^{2}}$, where $x y, x z, y z, x^{2}-y^{2}$, and $3 z^{2}-r^{2}$ correspond to $d_{1}, d_{2}, d_{3}, d_{4}$, and $d_{5}$, respectively (see eqs. (125) - (129)). Here, the relation of $E_{1}<E_{2}\left(=E_{3}\right)<E_{4}<E_{5}$ is considered, where the second excited states are doubly degenerate, i.e., $E_{2}=E_{3}$. In addition, $\Delta_{s}, \Delta_{m}$, and $\Delta_{l}$ represent $E_{2}-E_{1}, E_{4}-E_{1}$, and $E_{5}-E_{1}$, respectively.

of the down-spin electron, i.e.,

$$
\begin{aligned}
& \Psi_{j}=\psi_{j}=C_{j}\left(d_{j}+\sum_{m=1(\neq j)}^{5} c_{d_{m}}^{(j)} \overline{d_{m}}+\sum_{n=1}^{3} c_{p_{n}}^{(j)} \overline{p_{n}}\right), \\
& C_{j}=\left(1+\sum_{m=1(\neq j)}^{5}\left|c_{d_{m}}^{(j)}\right|^{2}+\sum_{n=1}^{3}\left|c_{p_{n}}^{(j)}\right|^{2}\right)^{-1 / 2} .
\end{aligned}
$$

Equation (36) also becomes

$$
\Phi_{j}=d_{j}
$$

The energy of $\Psi_{j}$ is given by $E_{j}=e_{j}+\Delta e_{j}$ (see eq. (45)), while that of $\Phi_{j}$ is $e_{j}$ (see eq. (38)). Here, $E_{j}$ is assumed to be $E_{j}$ in Fig. 2 by taking into account the crystal field of tetragonal symmetry. The wave functions $d_{j}$ and $p_{j}$ are then written as

$$
\begin{aligned}
& d_{1}=x y f_{3,2}(r), \\
& d_{2}=x z f_{3,2}(r), \\
& d_{3}=y z f_{3,2}(r), \\
& d_{4}=\frac{1}{2}\left(x^{2}-y^{2}\right) f_{3,2}(r), \\
& d_{5}=\frac{1}{2 \sqrt{3}}\left(3 z^{2}-r^{2}\right) f_{3,2}(r),
\end{aligned}
$$

and

$$
\begin{aligned}
& p_{1}=x f_{4,1}(r), \\
& p_{2}=y f_{4,1}(r), \\
& p_{3}=z f_{4,1}(r),
\end{aligned}
$$


respectively. The function $f_{n, l}(r)$ with the principal quantum number $n$ and the azimuthal quantum number $l$ is given by

$$
\begin{aligned}
& f_{3,2}(r)=\frac{2}{81 \sqrt{2 \pi} a_{\mathrm{B}, \mathrm{d}}^{7 / 2}} \exp \left(-\frac{r}{3 a_{\mathrm{B}, \mathrm{d}}}\right) \\
& f_{4,1}(r)=\frac{1}{512 \sqrt{5 \pi} a_{\mathrm{B}, \mathrm{p}}^{5 / 2}}\left[80-\frac{20 r}{a_{\mathrm{B}, \mathrm{p}}}+\left(\frac{r}{a_{\mathrm{B}, \mathrm{p}}}\right)^{2}\right] \exp \left(-\frac{r}{4 a_{\mathrm{B}, \mathrm{p}}}\right), \\
& a_{\mathrm{B}, \mathrm{d}}=\frac{a_{\mathrm{B}}}{Z_{\mathrm{eff}, \mathrm{d}}}, \\
& a_{\mathrm{B}, \mathrm{p}}=\frac{a_{\mathrm{B}}}{Z_{\mathrm{eff}, \mathrm{p}}}
\end{aligned}
$$

with $a_{\mathrm{B}}=\epsilon_{0} h^{2} /\left(\pi m e^{2}\right)$, where $a_{\mathrm{B}}$ is the Bohr radius and $Z_{\text {eff,d }}\left(Z_{\text {eff,p }}\right)$ is the effective nuclear charge for the $3 \mathrm{~d}$ electron (4p electron).

\subsection{Notation and parameter setup}

In this system, we first replace $\boldsymbol{r}_{t}=\left(x_{t}, y_{t}, z_{t}\right)$ and $\boldsymbol{p}_{t}=\left(p_{t, x}, p_{t, y}, p_{t, z}\right)$ with $(x, y, z)$ and $\left(p_{x}, p_{y}, p_{z}\right)$ of the one-electron system, respectively. The angular frequency $\omega_{\xi}$ is set to be $\omega_{x}=\omega_{y}=\omega_{z}=2 \pi f$, where $f$ is a vibration frequency. For eqs. (122) and (123), we put $c_{d_{m}}^{(j)}=c_{d}$ for $m=1$ - 5 with $m \neq j, c_{p_{n}}^{(j)}=c_{p}$ for $n=1-3$, and $C_{j}=\left(1+4 c_{d}^{2}+3 c_{p}^{2}\right)^{-1 / 2} \equiv C$. In addition, we use the notation of $e_{1}=e_{x y}, e_{2}=e_{x z}, e_{3}=e_{y z}, e_{4}=e_{x^{2}-y^{2}}, e_{5}=e_{3 z^{2}-r^{2}}, E_{1}=E_{x y}, E_{2}=E_{x z}, E_{3}=E_{y z}$, $E_{4}=E_{x^{2}-y^{2}}$, and $E_{5}=E_{3 z^{2}-r^{2}}$. The energy difference is then expressed as $E_{x z}-E_{x y}=E_{y z}-$ $E_{x y} \equiv \Delta_{s}(>0), E_{x^{2}-y^{2}}-E_{x y} \equiv \Delta_{m}(>0)$, and $E_{3 z^{2}-r^{2}}-E_{x y} \equiv \Delta_{l}(>0)$ (see Fig. 2). The energy difference $e_{1}-e_{j}=E_{1}-E_{j}$ is roughly set by taking into account $\left|\Delta e_{1}-\Delta e_{j}\right| \ll\left|e_{1}-e_{j}\right|$ for $j \neq 1$.

As a parameter set, we choose $c_{d}=c_{p}=0.15,{ }^{47} \Delta_{s}=0.45 \Delta_{m}, \Delta_{l}=1.45 \Delta_{m}, \Delta_{m}=0.5 \mathrm{eV}$, and $f=0.01 \mathrm{THz}-100 \mathrm{THz}$, which correspond to typical atomic vibration frequencies. ${ }^{48}$ The mass of $\mathrm{Fe}^{2+}, M$, is $M=(26+30) \times\left(1.67 \times 10^{-27}\right)+24 \times\left(9.11 \times 10^{-31}\right) \mathrm{kg}$, where the number of protons (neutrons) is $26(30)$, the mass of the proton (neutron) is $1.67 \times 10^{-27} \mathrm{~kg}\left(1.67 \times 10^{-27} \mathrm{~kg}\right)$, the number of electrons is 24 , and the electron mass is $9.11 \times 10^{-31} \mathrm{~kg}$. The effective nuclear charge $Z_{\text {eff,d }}\left(Z_{\text {eff,p }}\right)$ is evaluated to be $Z_{\text {eff,d }}=6.25\left(Z_{\text {eff,p }}=4.10\right)$ according to Slater's rules. ${ }^{37}$

Note that from now on we will use the coordinate system $x, y$, and $z$ for some coefficients in accordance with the notation in $\S 2$. Actually, however, $x=X, y=Y$, and $z=Z$ are realized in this system, as described in Appendix C.1.

\subsection{Frequency dependence of coefficients}

The coefficients of the spin-atomic vibration interaction are now written as $\Lambda_{\mu, \xi}^{(1)} / \lambda, \Lambda_{\mu, \xi}^{k} / \lambda$, $G_{\xi}, F / D, \Lambda_{I, J ; \xi}^{\prime} / D$, and $\Lambda_{\mu ; \nu, \xi}^{ \pm} / D$, while that of the spin-flip Hamiltonian is written as $\Lambda_{I, J}^{\mathrm{SF}} / D$. These expressions are dimensionless quantities. Note that $E$ of eq. (104) becomes zero owing to the tetragonal symmetry (see eq. (C·18)). The expressions of all the coefficients are described 


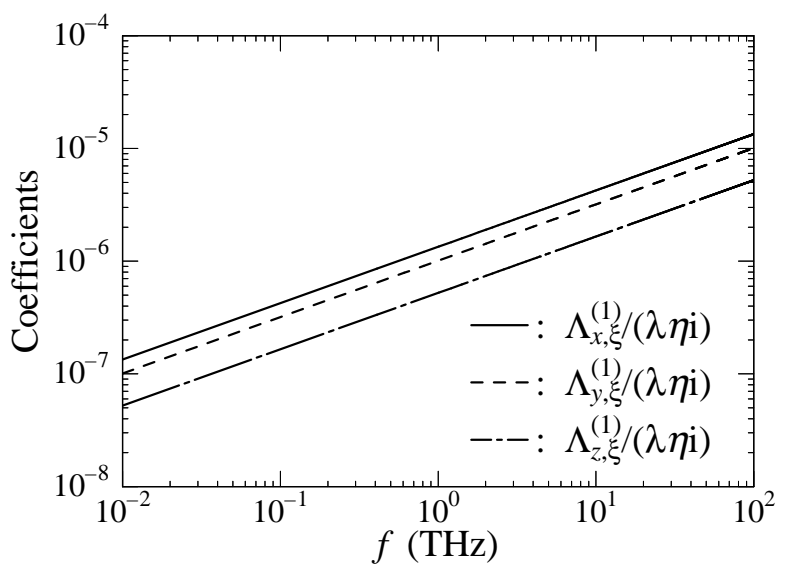

Fig. 3. Vibration frequency $f$ dependence of $\Lambda_{\mu, \xi}^{(1)} /(\lambda \eta \mathrm{i})$ for $\mu, \xi=x, y, z$. The parameters are set to be $c_{d}=c_{p}=0.15$ and $\Delta_{m}=0.5 \mathrm{eV}$.

in Appendix .

In Figs. 3 and 4 , we show the $f$ dependences of $\Lambda_{\mu, \xi}^{(1)} /(\lambda \eta \mathrm{i})$ and $\Lambda_{\mu, \xi}^{k} /(\lambda \eta i)$, respectively. These expressions are the coefficients of the $S_{I}\left(-a_{\xi}+a_{\xi}^{\dagger}\right)$ term. The magnitudes of the coefficients increase with increasing $f$, because they are proportional to $\sqrt{f}$.

Figures 5 - 9 show the $f$ dependences of $\Lambda_{I, J}^{\mathrm{SF}} / D, G_{\xi} / \eta, \Lambda_{I, J ; \xi}^{\prime} /(D \eta), \Lambda_{\mu ; \nu, \xi}^{+} /(D \eta)$, and $\Lambda_{\mu ; \nu, \xi}^{-} /(D \eta)$, respectively. These expressions are the coefficients of the $S_{I} S_{J} a_{\xi}$ and $S_{I} S_{J} a_{\xi}^{\dagger}$ terms. The coefficient $\Lambda_{I, J}^{\mathrm{SF}} / D$, which contains no $f$, certainly takes a constant value. The coefficients $G_{\xi} / \eta$ and $\left|\Lambda_{I, J ; \xi}^{\prime} /(D \eta)\right|$, which are proportional to $1 / \sqrt{f}$, decrease with increasing $f$. This behavior is in contrast to that of $\left|\Lambda_{\mu, \xi}^{(1)} /(\lambda \eta \mathrm{i})\right|$ and $\left|\Lambda_{\mu, \xi}^{k} /(\lambda \eta \mathrm{i})\right|$. The coefficient $\left|\Lambda_{\mu ; \nu, \xi}^{ \pm} /(D \eta)\right|$ monotonically decreases with increasing $f$ in a region of $f \lesssim 20 \mathrm{THz}$, because the dominant term of $\kappa_{u, \xi}^{ \pm}$in $\Lambda_{\mu, \nu, \xi}^{ \pm} / D$ (see eq. (C.80)) is i $\eta\left(m / \hbar^{2}\right) \sqrt{\hbar /\left(2 M \omega_{\xi}\right)} \Delta_{u}$, where $\omega_{\xi}=2 \pi f$. In the vicinity of $f=60 \mathrm{THz},\left|\Lambda_{\mu ; \nu, \xi}^{-} / D\right|$ drops, reflecting that some $\kappa_{u, \xi}^{-}$'s approach zero. Here, $\kappa_{u, \xi}^{-}=0$ reduces to $\hbar \omega_{\xi}=h f=\Delta_{u}$.

\subsection{Evaluation of coefficients}

For the evaluation of the coefficients, we here consider a model in which the Fe ion $\left(3 \mathrm{~d}^{6}\right)$ in the crystal field of tetragonal symmetry vibrates in the $x$ direction. The spin-orbit coupling constant $\lambda$ is assumed to be $\lambda=-5,-10,-25$, and $-40 \mathrm{meV}$, where $\lambda$ of $\mathrm{Fe}^{2+}$ has been previously evaluated to be $-12.6 \mathrm{meV} .{ }^{35}$ The anisotropy constant $D$ can then be evaluated by substituting the above $\lambda$ 's, $c_{d}=c_{p}=0.15, \Delta_{m}=0.5 \mathrm{eV}$, and $\Delta_{s}=0.45 \Delta_{m}$ into eq. (C.17). Namely, we have $D=-6.63 \times 10^{-2} \mathrm{meV}$ for $\lambda=-5 \mathrm{meV}, D=-2.65 \times 10^{-1} \mathrm{meV}$ for $\lambda=-10$ $\mathrm{meV}, D=-1.66 \mathrm{meV}$ for $\lambda=-25 \mathrm{meV}$, and $D=-4.25 \mathrm{meV}$ for $\lambda=-40 \mathrm{meV}$. We also use $\eta=0.05$, which is evaluated in Appendix .

Using the above $\lambda$ 's and $D$ 's and the results in Figs. 3 - 9, we can evaluate the coefficients of $V_{\mathrm{SA}}$ and $V_{\mathrm{SF}}$. In particular, we focus on large components in the respective coefficients, i.e., 

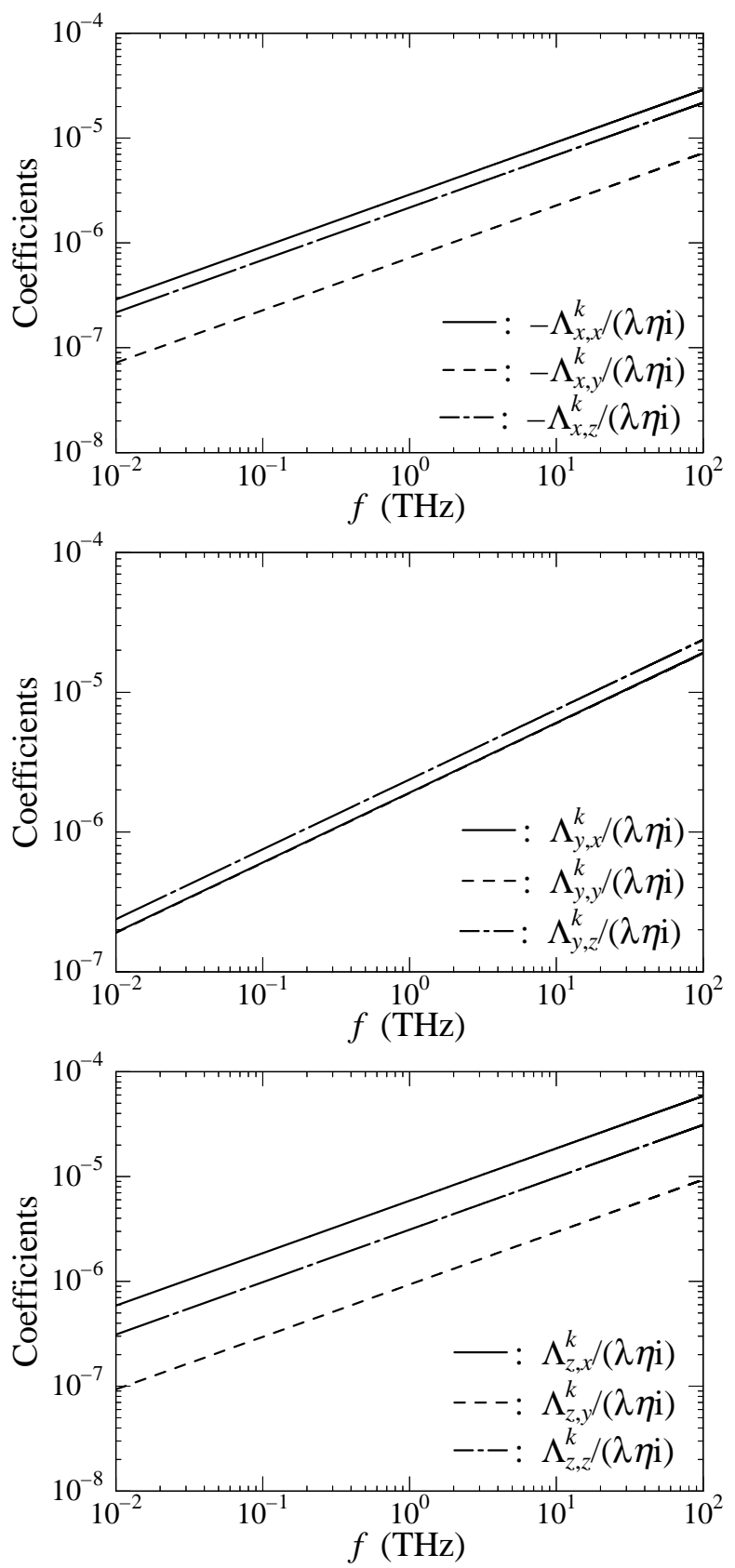

Fig. 4. Vibration frequency $f$ dependences of the coefficients. Upper panel: $\Lambda_{x, \nu}^{k} /(\lambda \eta \mathrm{i})$ for $\nu=x, y, z$. Middle panel: $\Lambda_{y, \nu}^{k} /(\lambda \eta \mathrm{i})$ for $\nu=x, y, z$. Lower panel: $\Lambda_{z, \nu}^{k} /(\lambda \eta \mathrm{i})$ for $\nu=x, y, z$. Note that $\Lambda_{y, x}^{k} /(\lambda \eta \mathrm{i})$ takes almost the same value as $\Lambda_{y, y}^{k} /(\lambda \eta \mathrm{i})$ at each $f$. The parameters are set to be $c_{d}=c_{p}=0.15$ and $\Delta_{m}=0.5 \mathrm{eV}$.

$\Lambda_{y, x}^{(1)}, \Lambda_{z, x}^{k}, G_{x} D, G_{x} F, \Lambda_{X, Z ; x}^{\prime}, \Lambda_{Y, Z ; x}^{\prime}$, and $\Lambda_{z ; z, x}^{ \pm}$in $V_{\mathrm{SA}}$, and $\Lambda_{X, Z}^{\mathrm{SF}}$ and $\Lambda_{Y, Z}^{\mathrm{SF}}$ in $V_{\mathrm{SF}}$. In the upper and lower panels of Fig. 10, we show the $f$ dependences of the coefficients with $\lambda=-5$ and $-10 \mathrm{meV}$, respectively. In addition, the upper and lower panels of Fig. 11 show those with $\lambda=-25$ and $-40 \mathrm{meV}$, respectively.

We first find that the magnitudes of the coefficients of $V_{\mathrm{SF}}$ are larger than those of the 
J. Phys. Soc. Jpn.

Full PAPER

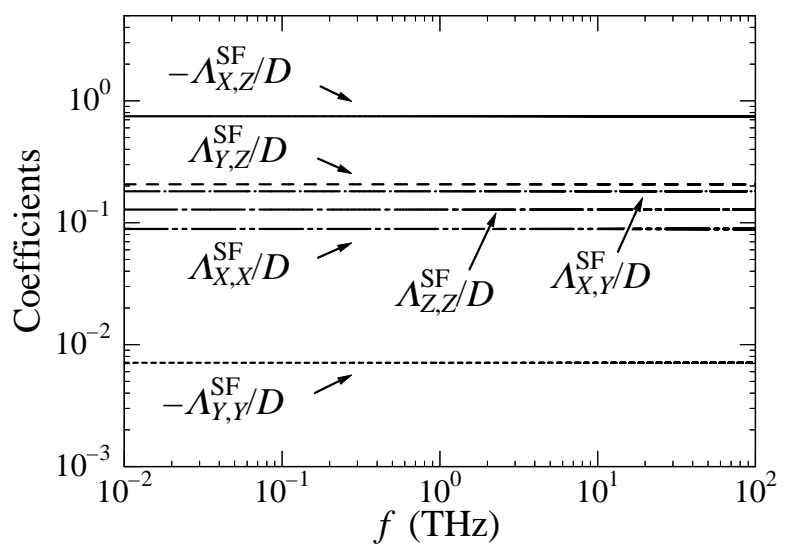

Fig. 5. Vibration frequency $f$ dependence of $\Lambda_{I, J}^{\mathrm{SF}} / D$ for $I, J=X, Y, Z$, where $\Lambda_{I, J}^{\mathrm{SF}}=\Lambda_{J, I}^{\mathrm{SF}}$ (see $\S 3.4 .1$ ). The coefficients take the respective constant values. The parameters are set to be $c_{d}=c_{p}=0.15$ and $\Delta_{m}=0.5 \mathrm{eV}$.

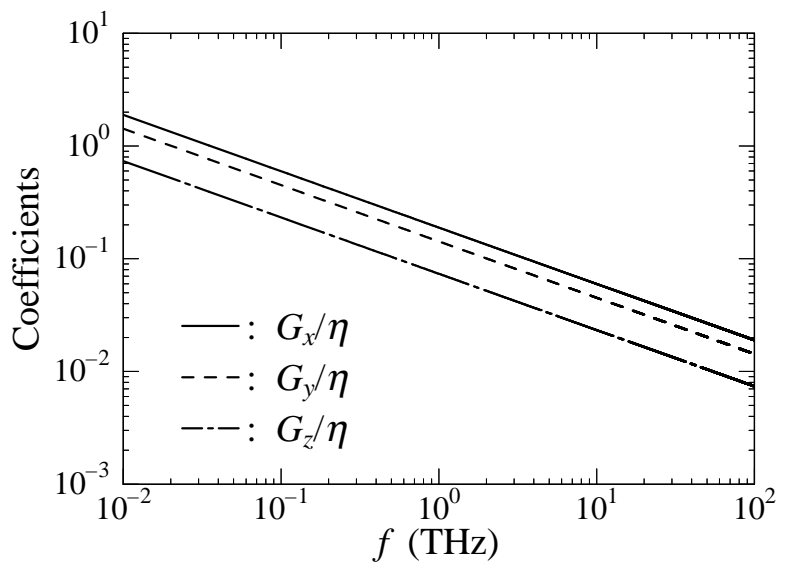

Fig. 6. Vibration frequency $f$ dependence of $G_{\xi} / \eta$ for $\xi=x, y, z$. The parameters are set to be $c_{d}=c_{p}=0.15$ and $\Delta_{m}=0.5 \mathrm{eV}$.

$V_{\mathrm{SA}}$. This relation mainly indicates that the coefficients of $V_{\mathrm{SA}}$ are proportional to $\eta$ (i.e., the degree of the difference in vibration displacement), whereas $V_{\mathrm{SF}}$ does not contain $\eta$. As found from eqs. (111) and (106), the major difference between $\Lambda_{I, J ; \xi}^{\prime}$ and $\Lambda_{I, J}^{\mathrm{SF}}$ may be the presence or absence of $\eta$.

In $V_{\mathrm{SA}}$, " $\left|G_{x} D\right|,\left|G_{x} F\right|,\left|\Lambda_{X, Z ; x}^{\prime}\right|$, and $\left|\Lambda_{Y, Z ; x}^{\prime}\right|$ " tend to be larger than $\left|\Lambda_{z, z ; x}^{ \pm}\right|$and $\left|\Lambda_{z, x}^{k}\right|$, because of the relation of $\left|V_{\mathrm{so} 1}\right| \gg\left|V_{\mathrm{so} 2}\right|,\left|V_{\mathrm{k}}^{\prime}\right|$ (see eqs. (63) - (65)). In particular, the relation of $\left|V_{\mathrm{so} 1}\right| \gg\left|V_{\mathrm{so} 2}\right|$ indicates that the rate of the change in $\lambda$ due to the vibration, $|\Delta \lambda / \lambda|$, is larger than that in $\boldsymbol{L}$ due to the vibration, $|\Delta \boldsymbol{L} / \boldsymbol{L}|$, in a region with an atomic radius of $r \sim 0.1 \mathrm{~nm}$ (see eqs. (30) and (31)). Here, $\Delta \lambda$ and $\Delta \boldsymbol{L}$ are given by $\Delta \lambda=$ $\eta C_{ \pm}\left\langle\partial r^{-3} / \partial x\right\rangle\left(-\Delta x_{\mathrm{n}}\right)=\eta C_{ \pm}\left\langle 3 x r^{-5}\right\rangle \Delta x_{\mathrm{n}}$ and $\Delta \boldsymbol{L}=-(\eta / \hbar)\left(\boldsymbol{r}_{t} \times \Delta \boldsymbol{p}_{\mathrm{e}}+\Delta \boldsymbol{r}_{\mathrm{n}} \times \boldsymbol{p}_{t}\right)$, respectively, where $\Delta \boldsymbol{p}_{\mathrm{e}}=\left(\Delta p_{\mathrm{e}, x}, 0,0\right)$ and $\Delta \boldsymbol{r}_{\mathrm{n}}=\left(\Delta x_{\mathrm{n}}, 0,0\right)$ are set in this section. 

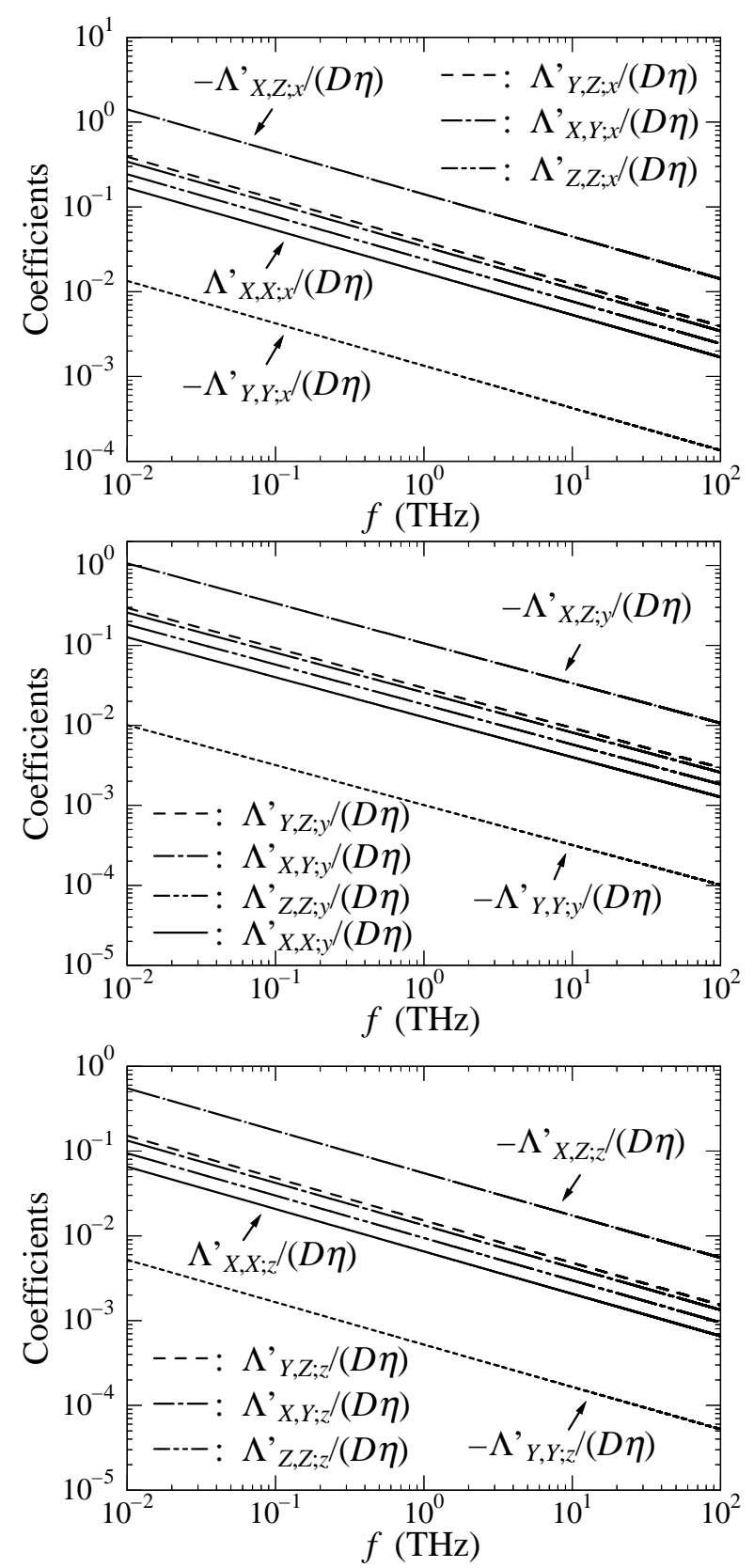

Fig. 7. Vibration frequency $f$ dependences of the coefficients. Upper panel: $\Lambda_{I, J ; x}^{\prime} /(D \eta)$ for $I, J=X$, $Y, Z$. Middle panel: $\Lambda_{I, J ; y}^{\prime} /(D \eta)$ for $I, J=X, Y, Z$. Lower panel: $\Lambda_{I, J ; z}^{\prime} /(D \eta)$ for $I, J=X, Y, Z$. We have $\Lambda_{I, J ; \xi}^{\prime}=\Lambda_{J, I ; \xi}^{\prime}($ see $\S 3.4 .2)$. The parameters are set to be $c_{d}=c_{p}=0.15$ and $\Delta_{m}=0.5 \mathrm{eV}$.

\section{Comparison between $V_{\mathrm{SA}}$ and the Conventional Spin-Phonon Interaction}

In this section, we compare $V_{\mathrm{SA}}$ with the conventional spin-phonon interactions derived by Mattuck and Strandberg ${ }^{25}$ and by Hartmann-Boutron et al. ${ }^{30}$ In particular, we focus on the $f$ dependences and magnitudes of the coefficients in the case of the Fe ion. We also use $\eta=0.05$, which is evaluated in Appendix . 

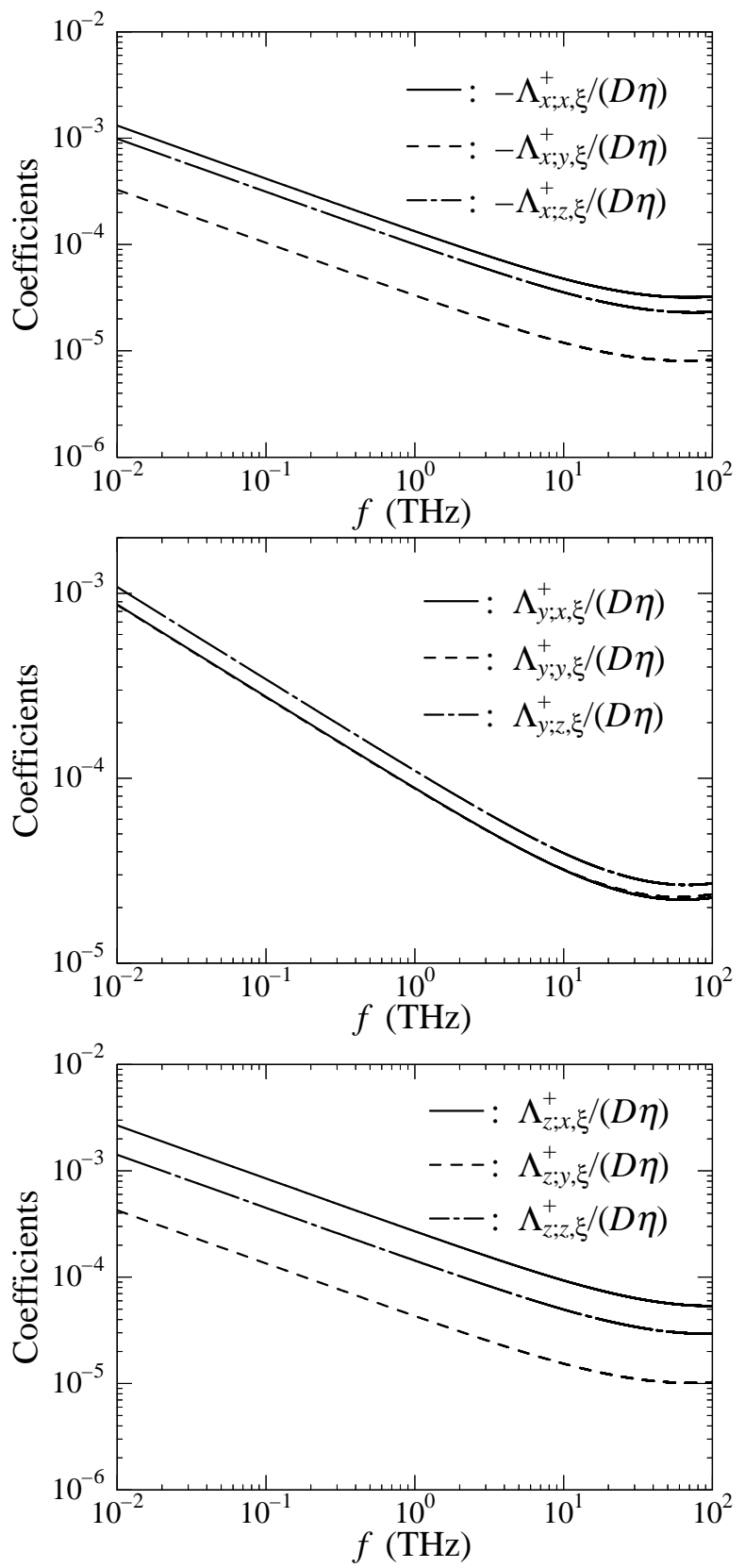

Fig. 8. Vibration frequency $f$ dependences of the coefficients. Upper panel: $\Lambda_{x ; \nu, \xi}^{+} /(D \eta)$ for $\nu, \xi=x$, $y, z$. Middle panel: $\Lambda_{y ; \nu, \xi}^{+} /(D \eta)$ for $\nu, \xi=x, y, z$. Lower panel: $\Lambda_{z ; \nu, \xi}^{+} /(D \eta)$ for $\nu, \xi=x, y, z$. We note that $\Lambda_{y ; x, \xi}^{+} /(D \eta)$ takes almost the same value as $\Lambda_{y ; y, \xi}^{+} /(D \eta)$ at each $f$. The parameters are set to be $c_{d}=c_{p}=0.15$ and $\Delta_{m}=0.5 \mathrm{eV}$.

\subsection{Modification of $V_{\mathrm{SA}}$}

To compare $V_{\mathrm{SA}}$ with the conventional spin-phonon interaction (see $\S 5.2$ ), we first modify $V_{\mathrm{SA}}$ to a novel spin-phonon interaction. We here consider the following one-dimensional lattice system: ions with the same mass $M$ are placed at even intervals along the $x$ axis, and vibrate in the $x$ direction. In addition, a specific ion located at the origin possesses $\boldsymbol{S}$, 

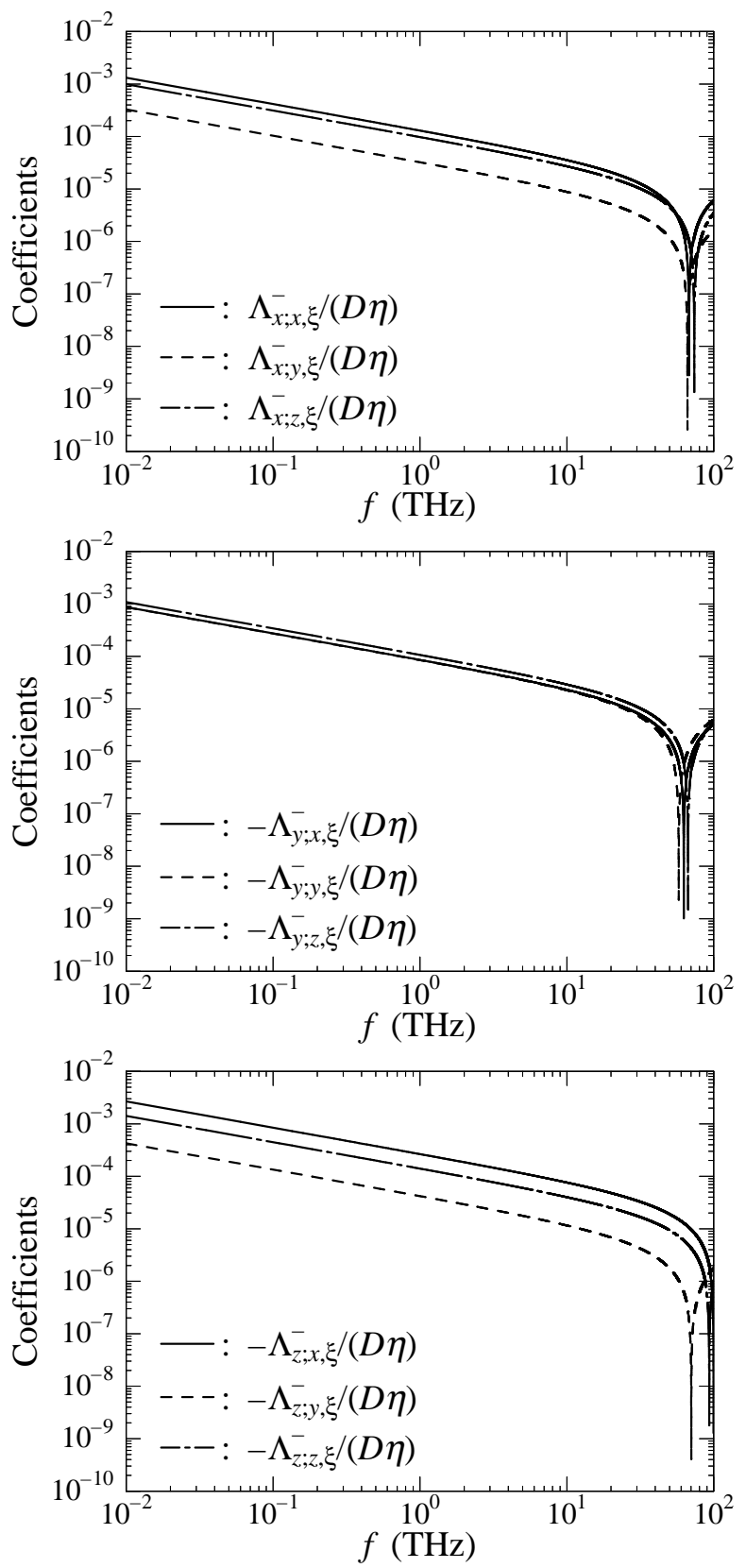

Fig. 9. Vibration frequency $f$ dependences of the coefficients. Upper panel: $\Lambda_{x ; \nu, \xi}^{-} /(D \eta)$ for $\nu, \xi=x$, $y, z$. Middle panel: $\Lambda_{y ; \nu, \xi}^{-} /(D \eta)$ for $\nu, \xi=x, y, z$. Lower panel: $\Lambda_{z ; \nu, \xi}^{-} /(D \eta)$ for $\nu, \xi=x, y, z$. We note that $\Lambda_{y ; x, \xi}^{-} /(D \eta)$ takes almost the same value as $\Lambda_{y ; y, \xi}^{-} /(D \eta)$ at each $f$. The parameters are set to be $c_{d}=c_{p}=0.15$ and $\Delta_{m}=0.5 \mathrm{eV}$.

and also this ion is surrounded by several negative ions. The system may be just described by a simple model in which $S$ located at the origin is coupled to the phonon for the lattice system. Thus, $V_{\mathrm{SA}}$ in this model is obtained by replacing $\Delta x_{\mathrm{n}}$ in eq. (56) and $\Delta p_{\mathrm{n}, x}$ in eq. (58) with $(1 / \sqrt{N}) \sum_{q} \sqrt{\hbar /\left(2 M \omega_{q}\right)}\left(a_{q}+a_{q}^{\dagger}\right)$ and $(1 / \sqrt{N}) \sum_{q} \mathrm{i} \sqrt{M \hbar \omega_{q} / 2}\left(-a_{q}+a_{q}^{\dagger}\right)$, respectively. Here, $N$ is the number of unit cells, $q$ is the wave vector, $\omega_{q}$ is the angular frequency 
J. Phys. Soc. Jpn.

FULL PAPER
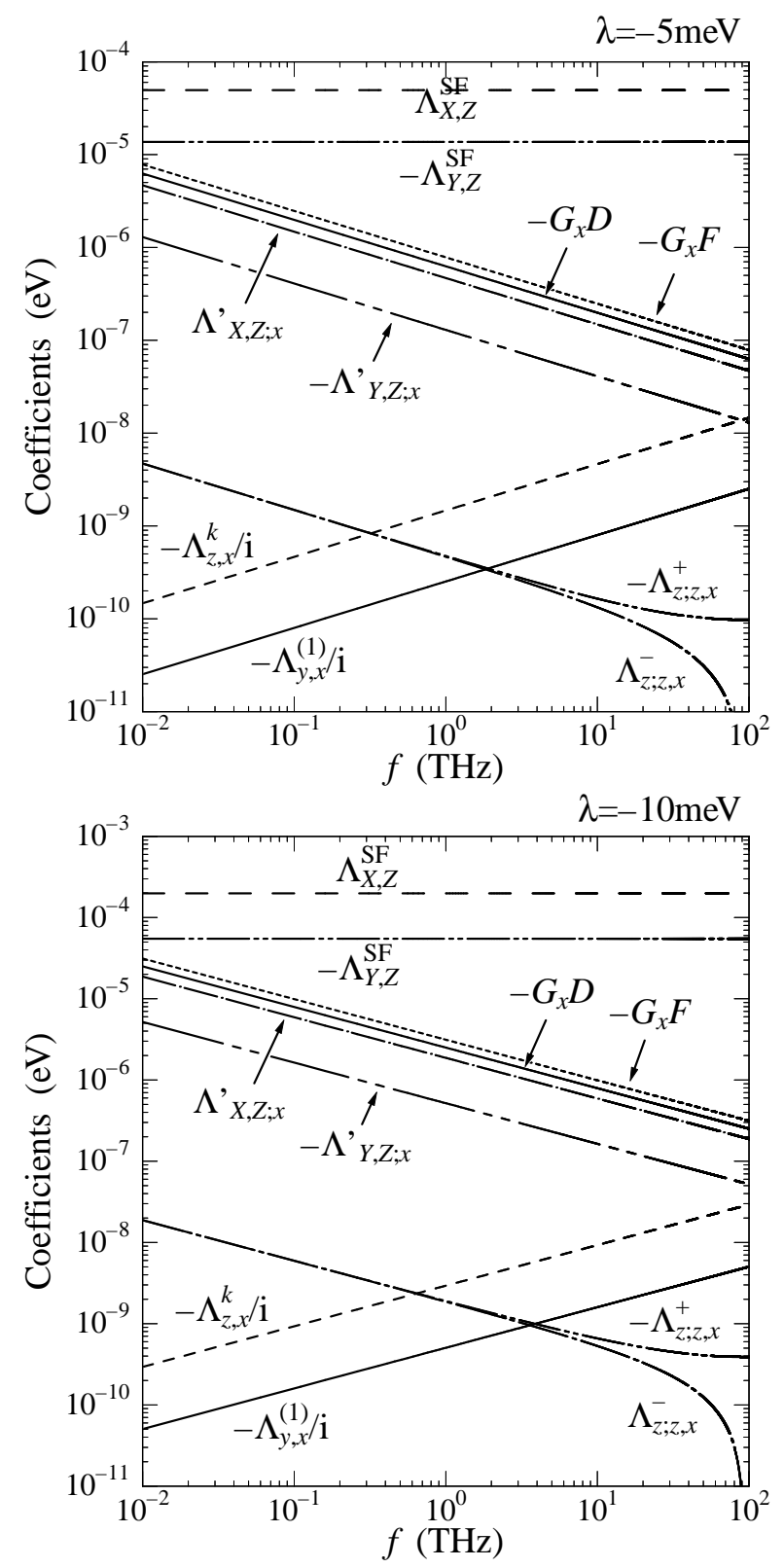

Fig. 10. Vibration frequency $f$ dependences of $\Lambda_{y, x}^{(1)}, \Lambda_{z, x}^{k}, G_{x} D, G_{x} F, \Lambda_{X, Z ; x}^{\prime}, \Lambda_{Y, Z ; x}^{\prime}$, and $\Lambda_{z ; z, x}^{ \pm}$in $V_{\mathrm{SA}}$, and $\Lambda_{X, Z}^{\mathrm{SF}}$ and $\Lambda_{Y, Z}^{\mathrm{SF}}$ in $V_{\mathrm{SF}}$. Upper panel: $\lambda=-5 \mathrm{meV}$. Lower panel: $\lambda=-10 \mathrm{meV}$. The cases of $\lambda=-5$ and $-10 \mathrm{meV}$ lead to $D=-6.63 \times 10^{-2}$ and $-2.65 \times 10^{-1} \mathrm{meV}$, respectively. The parameters are set to be $\eta=0.05, c_{d}=c_{p}=0.15$, and $\Delta_{m}=0.5 \mathrm{eV}$.

of the phonon at $q$, and $a_{q}\left(a_{q}^{\dagger}\right)$ is the annihilation operator (creation operator) of the phonon with $q$. As a result, $V_{\mathrm{SA}}$ of eq. (120) is modified as follows:

$$
\begin{aligned}
V_{\mathrm{SA}}= & \sum_{I=X, Y, Z} S_{I} \frac{1}{\sqrt{N}} \sum_{q} c_{I, x, q}\left(-a_{q}+a_{q}^{\dagger}\right) \\
& +\sum_{I, J=X, Y, Z} S_{I} S_{J} \frac{1}{\sqrt{N}} \sum_{q}\left(c_{1, I, J, x, q} a_{q}+c_{2, I, J, x, q} a_{q}^{\dagger}\right)
\end{aligned}
$$


J. Phys. Soc. Jpn.

FULL PAPER
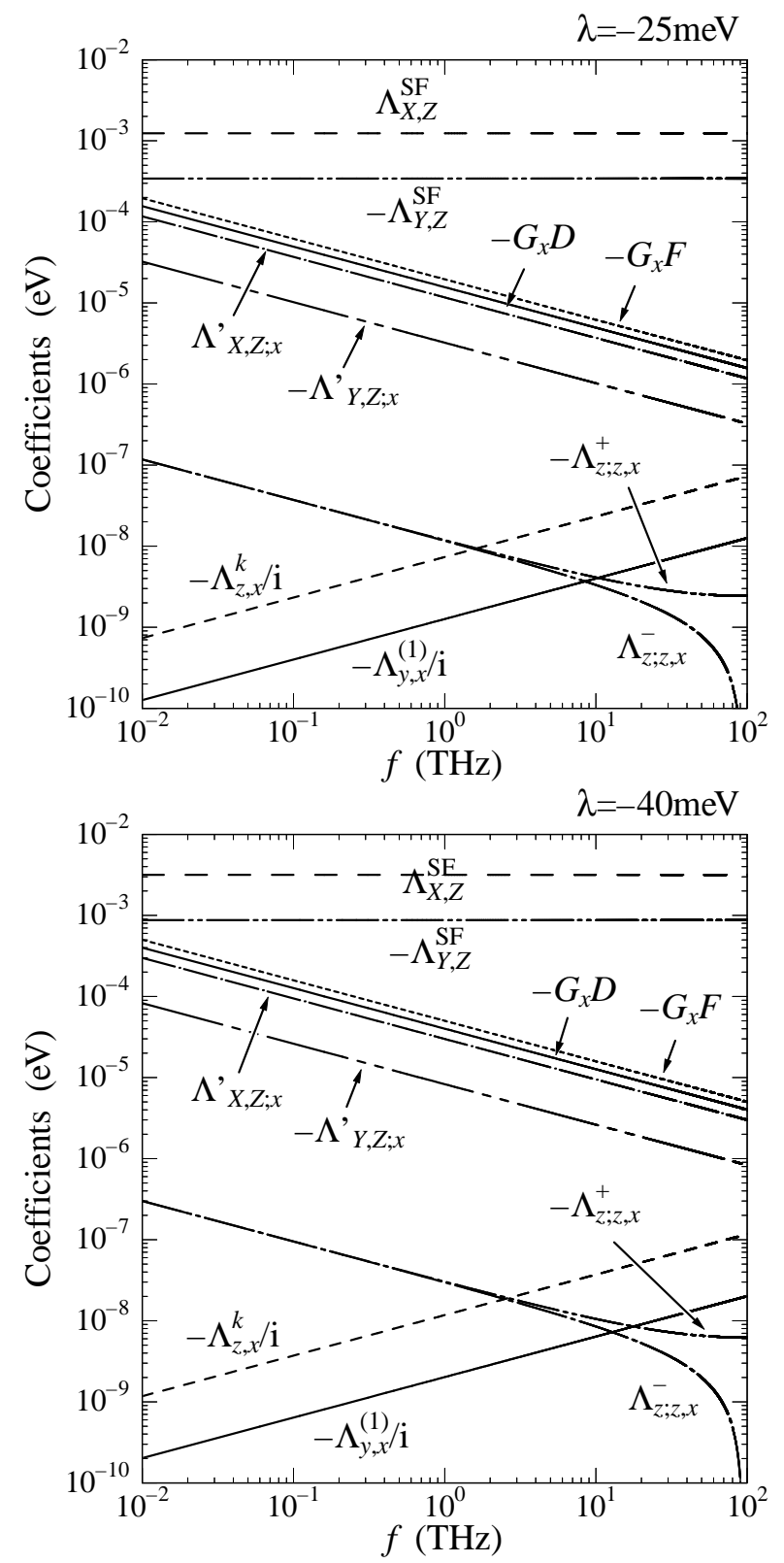

Fig. 11. Vibration frequency $f$ dependences of $\Lambda_{y, x}^{(1)}, \Lambda_{z, x}^{k}, G_{x} D, G_{x} F, \Lambda_{X, Z ; x}^{\prime}, \Lambda_{Y, Z ; x}^{\prime}$, and $\Lambda_{z ; z, x}^{ \pm}$in $V_{\mathrm{SA}}$, and $\Lambda_{X, Z}^{\mathrm{SF}}$ and $\Lambda_{Y, Z}^{\mathrm{SF}}$ in $V_{\mathrm{SF}}$. Upper panel: $\lambda=-25 \mathrm{meV}$. Lower panel: $\lambda=-40 \mathrm{meV}$. The cases of $\lambda=-25$ and $-40 \mathrm{meV}$ lead to $D=-1.66$ and $-4.25 \mathrm{meV}$, respectively. The parameters are set to be $\eta=0.05, c_{d}=c_{p}=0.15$, and $\Delta_{m}=0.5 \mathrm{eV}$.

$$
+\left[D S_{Z}^{2}+F S(S+1)\right] \frac{1}{\sqrt{N}} \sum_{q} G_{x, q}\left(a_{q}+a_{q}^{\dagger}\right) .
$$

The coefficients $c_{I, x, q}, c_{1, I, J, x, q}, c_{2, I, J, x, q}$, and $G_{x, q}$, respectively, correspond to $c_{I, x}, c_{1, I, J, x}$, $c_{2, I, J, x}$, and $G_{x}$ in eq. (120), where $\omega_{x}$ in these coefficients has been replaced with $\omega_{q}$.

We now replace $\omega_{q}$ with $2 \pi f$ in order to investigate the $f$ dependence of the coefficients. We then can regard $c_{I, x, q}, c_{1, I, J, x, q}, c_{2, I, J, x, q}$, and $G_{x, q}$ as $c_{I, x}, c_{1, I, J, x}, c_{2, I, J, x}$, and $G_{x}$ in eq. 


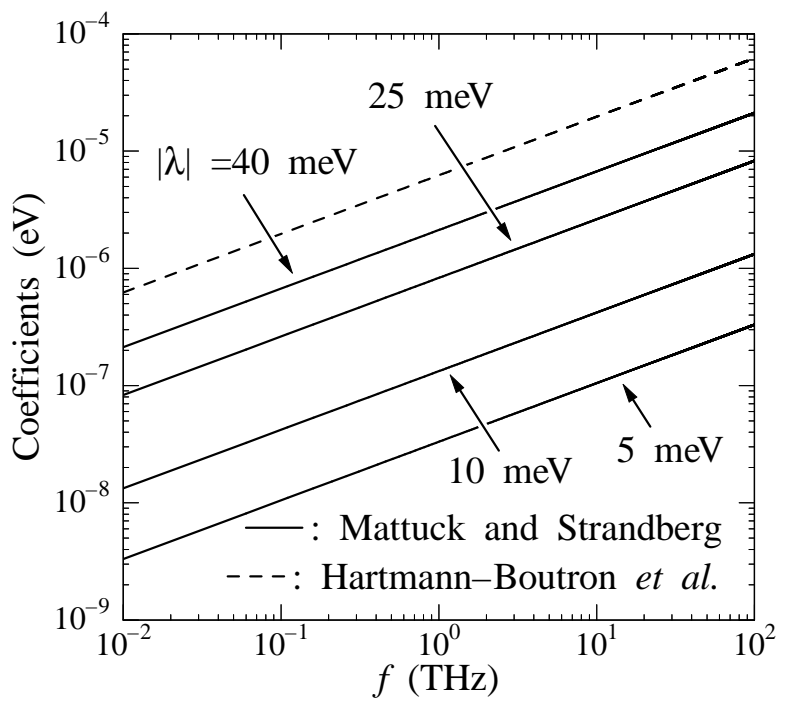

Fig. 12. Vibration frequency $f$ dependence of the magnitude of coefficient of the spin-phonon inter-

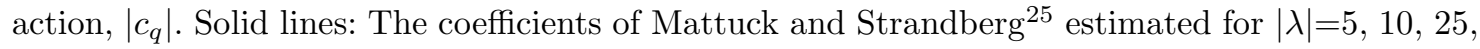
and $40 \mathrm{meV}$. Dotted line: The coefficient of Hartmann-Boutron et al. ${ }^{30}$ In this coefficient, $\lambda$ is unspecified.

(120), respectively, where $\omega_{x}=2 \pi f$. Such coefficients may be directly compared with those of the spin-phonon interaction of the below-mentioned eqs. (138), (139), and (140). In particular, we focus on large components in the respective coefficients in the case of the Fe ion $\left(3 \mathrm{~d}^{6}\right)$ in the crystal field of tetragonal symmetry, i.e., $\Lambda_{y, x}^{(1)}, \Lambda_{z, x}^{k}, G_{x} D, G_{x} F, \Lambda_{X, Z ; x}^{\prime}, \Lambda_{Y, Z ; x}^{\prime}$, and $\Lambda_{z ; z, x}^{ \pm}$ (see Figs. 10 and 11).

\subsection{Spin-phonon interaction}

The spin-phonon interaction originally represents an interaction between a single spin located at the origin and the phonon of a lattice system. ${ }^{25,30}$ Here, a unit cell of the lattice consists of a magnetic ion (with a single spin) and the surrounding ions. In addition, all the ions vibrate. Note that exchange interactions between the spins are neglected.

As a similar lattice system to that in $\S 5.1$, a one-dimensional lattice system is considered, in which each unit cell is aligned along the $x$ axis. In addition, all the ions vibrate in the $x$ direction. The dominant term in the spin-phonon interaction, ${ }^{25,30} V_{\mathrm{SP}}$, is then expressed as

$$
V_{\mathrm{SP}}=\sum_{I, J=X, Y, Z} S_{I} S_{J} \frac{1}{\sqrt{N}} \sum_{q}\left(c_{q} a_{q}+c_{q}^{*} a_{q}^{\dagger}\right),
$$

where $N$ is the number of unit cells, $q$ is the wave vector, and $c_{q}$ is the coefficient.

The coefficient of $V_{\mathrm{SP}}$ derived by Mattuck and Strandberg, ${ }^{25} c_{q}$, is written as

$$
c_{q}=\frac{1}{4 \pi \epsilon_{0}} \sqrt{\frac{\hbar \omega_{q}}{2 M_{\mathrm{u}} v^{2}}} \frac{6 e^{2} r_{0}^{2}}{\Delta^{2} R^{3}} \lambda^{2},
$$

with $\omega_{q}=2 \pi f_{q} \cdot{ }^{49}$ Here, $\lambda$ is the spin-orbit coupling constant of eq. (7), $\Delta$ is the energy splitting 
under the crystal field, $R$ is the distance between the magnetic ion and the surrounding ions, $r_{0}$ is the ionic radius, $M_{\mathrm{u}}$ is the mass per unit cell, and $v$ is the phonon velocity defined as $v=\omega_{q} /|q|$, where $v$ is constant. This spin-phonon interaction is essentially the same as the expression by Van Vleck, ${ }^{23}$ which corresponds to the third-order perturbation energy. Here, the crystal field potential energy is the unperturbed term, while $V_{\mathrm{so} 0}$ of eq. (29) and the modulation of crystal field potential energy due to the lattice vibration are the perturbed terms. ${ }^{23,29}$ We mention that the second-order perturbation energy due to $V_{\text {so0 }}$ and the modulation of crystal field potential energy vanishes owing to the Van Vleck cancellation. ${ }^{23,45}$

To estimate $c_{q}$, we assume $\Delta=1 \mathrm{eV}, R=1 \mathrm{~nm}$, and $r_{0}=0.1 \mathrm{~nm}$. We also use $M_{\mathrm{u}}=[(26+30)+5 \times(29+34)+2 \times(7+7)] \times\left(1.67 \times 10^{-27}\right)+(26+5 \times 29+2 \times 7) \times\left(9.11 \times 10^{-31}\right) \mathrm{kg}$ on the basis of results of the STM experiments for the Fe ion on the CuN surface. ${ }^{10}$ The unit cell has been assumed here to consist of one Fe ion, five $\mathrm{Cu}$ ions, and two $\mathrm{N}$ ions. The numbers of protons (the number of neutrons) of Fe is 26 (30), that of protons (that of neutrons) of $\mathrm{Cu}$ is 29 (34), and that of protons (that of neutrons) of $\mathrm{N}$ is 7 (7). The numbers of electrons of $\mathrm{Fe}, \mathrm{Cu}$, and $\mathrm{N}$ neutral atoms are 26, 29, and 7, respectively. Since the numerical value of $v$ is unknown for this system, we utilize $v=1.45 \times 10^{3} \mathrm{~m} / \mathrm{s},{ }^{50}$ which is the value evaluated for $\mathrm{Mn}_{12}$.

On the other hand, the coefficient of $V_{\mathrm{SP}}$ derived by Hartmann-Boutron et al. ${ }^{30} c_{q}$ is given by

$$
c_{q}=\mathrm{i} \sqrt{\frac{\hbar \omega_{q}}{2 M_{\mathrm{u}} v^{2}}} V_{q}
$$

with $\omega_{q}=2 \pi f_{q}$. Here, $q=\omega_{q} / v$ has been used, where $v$ is constant. The quantity $V_{q}$ is regarded as the coefficient with a unit of energy. ${ }^{6}$ This interaction originally arises from the spin-orbit interaction and the local strain at the spin site due to the phonon. To estimate $c_{q}$, we use $\left|V_{q}\right|=4.45 \mathrm{meV}$ and the above-mentioned $M_{\mathrm{u}}$ and $v$. This $\left|V_{q}\right|$ is obtained from $C_{\text {pre }}=3\left|V_{q}\right|^{2} /\left(2 \pi \hbar^{4} \rho v^{5}\right),{ }^{1}$ with $\rho=M_{\mathrm{u}} / a^{3}$, where the volume of the unit cell, $a^{3}$, is set to be $a^{3}=1 \mathrm{~nm}^{3}$. The quantity $C_{\text {pre }}$ corresponds to a prefactor of the expression of the relaxation rate, and then $C_{\mathrm{pre}}=10^{4} \mathrm{~Hz} / \mathrm{K}^{3}$ is chosen on the basis of experimental results for magnetic ions. $^{1,51}$

\subsection{Comparison}

We first make a comparison of the $f$ dependence of the coefficients between $V_{\mathrm{SP}}$ and $V_{\mathrm{SA}}$. Figure 12 shows the $f$ dependence of $\left|c_{q}\right|$ of eqs. (139) and (140), where $f$ is defined by $f=f_{q}$. We find that $\left|c_{q}\right|$ is proportional to $\sqrt{f}$. Such $f$ dependence (i.e., $\sqrt{f}$ ) is different from those

of the coefficients of the $S_{I} S_{J} a_{q}$ and $S_{I} S_{J} a_{q}^{\dagger}$ terms of $V_{\mathrm{SA}}$ (i.e., $1 / \sqrt{f}$ ), but the same as that of the $S_{I}\left(-a_{q}+a_{q}^{\dagger}\right)$ term of $V_{\mathrm{SA}}$.

Next, as seen from Figs. $10-12,\left|G_{x} D\right|,\left|G_{x} F\right|,\left|\Lambda_{X, Z: x}^{\prime}\right|$, and $\left|\Lambda_{Y, Z: x}^{\prime}\right|$ in $V_{\mathrm{SA}}$ are larger than $\left|c_{q}\right|$ in the low $f$ region. For example, when $\lambda=-10,-25$, and $-40 \mathrm{meV}$, the former are 
larger than the latter in a region of $f \lesssim 1 \mathrm{THz}$. Also, the region of $f$, in which the former become larger than the latter, expands with increasing $\lambda$.

As the main reason why the magnitudes of the coefficients of $V_{\mathrm{SA}}$ can be larger than $\left|c_{q}\right|$ of Mattuck and Strandberg, we consider that $M$ in the coefficients of $V_{\mathrm{SA}}\left(\propto M^{-1 / 2}\right)$ is smaller than $M_{\mathrm{u}}$ in $c_{q}$ of $V_{\mathrm{SP}}\left(\propto M_{u}{ }^{-1 / 2}\right)$. Originally, the decrease in $M$ increases the magnitude of vibration displacement $\left|\Delta \xi_{\mathrm{n}}\right|(\xi=x, y, z)$ (see eq. (56) and Appendix ). Furthermore, the increase in $\left|\Delta \xi_{\mathrm{n}}\right|$ enhances the magnitude of $V_{\mathrm{SA}}$ (i.e., $v_{\mathrm{so} 0, \mathrm{so1}, 2}^{(2)}$ ) (e.g., eqs. (63) and (30)).

On the other hand, $c_{q}$ of Hartmann-Boutron et al. is rewritten as $c_{q}=\mathrm{i} \sqrt{\hbar^{5} \omega_{q} v^{3} C_{\text {pre }} /\left(3 a^{3}\right)}$, by substituting the above-mentioned $\left|V_{q}\right|^{2}=\left(2 \pi \hbar^{4} / 3\right)\left(M_{\mathrm{u}} / a^{3}\right) v^{5} C_{\text {pre }}$ into eq. (140). Since the related quantities of $v, a$, and $C_{\text {pre }}$ in $c_{q}$ are not contained in $V_{\mathrm{SA}}$, it may be impossible to identify the cause of the larger magnitudes of the coefficients of $V_{\mathrm{SA}}$ than $\left|c_{q}\right|$.

We anticipate that at a low $f$, the $1 / \sqrt{f}$ terms in $V_{\mathrm{SA}}$ can be verified from the pressure effect of the spin relaxation time, and so on.

\section{Transition Probability per Unit Time}

In this section, we discuss transition probabilities per unit time due to $V_{\mathrm{SA}}$ and $V_{\mathrm{SF}}$ for a simple model of the Fe ion with bistable states. Our interests are in how the expressions of $V_{\mathrm{SA}}$ (e.g., $\left.S_{X} S_{Z}\left(a+a^{\dagger}\right)\right)$ and $V_{\mathrm{SF}}$ (e.g., $\left.S_{X} S_{Z}\right)$ influence the transition probabilities. Note also that the transition probabilities will be necessary for investigating spin relaxation phenomena. ${ }^{19}$

When $\mathcal{V}_{i}$ is considered to be a time-dependent perturbed Hamiltonian, we have the probability per unit time of a transition from a state $|u\rangle$ to $|v\rangle, W_{u \rightarrow v}^{i}$ :

$$
W_{u \rightarrow v}^{i}=\frac{2 \pi}{\hbar}\left|\left\langle v\left|\mathcal{V}_{i}\right| u\right\rangle\right|^{2} \frac{1}{\pi} \frac{\hbar / \tau}{\left(E_{u}-E_{v}\right)^{2}+(\hbar / \tau)^{2}},
$$

with $\mathcal{H}_{0}^{\mathrm{AS}}|u\rangle=E_{u}|u\rangle$, where $\mathcal{H}_{0}^{\mathrm{AS}}$ is the unperturbed Hamiltonian for the atomic spin, and $|u\rangle$ is the eigenstate of $\mathcal{H}_{0}^{\mathrm{AS}} .{ }^{52}$ Here, a correlation time for $\mathcal{V}_{i}, \tau$, is introduced by assuming that the correlation function for $\mathcal{V}_{i}$ has an exponential decay. In addition, the case of $E_{u}=E_{v}$ represents the conservation of energy for a transition from $|u\rangle$ to $|v\rangle$, while the case of $E_{u} \neq E_{v}$ represents the nonconservation of energy for the transition.

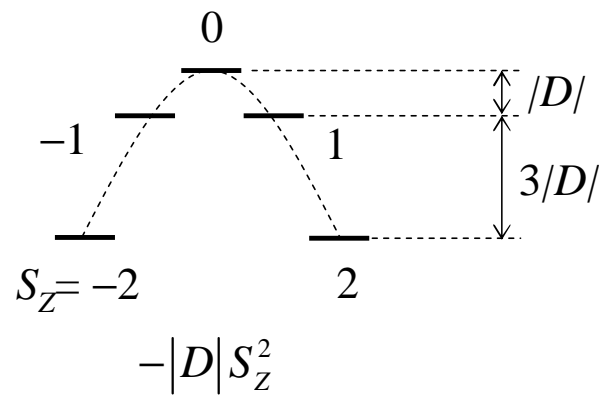

Fig. 13. Energy levels of $-|D| S_{Z}^{2}$ with $S=2$. 
J. Phys. Soc. Jpn.

Full PAPER

We now propose $\mathcal{H}_{0}^{\mathrm{AS}}$ and $\mathcal{V}_{i}$ of a simple model for the Fe ion in the crystal field of tetragonal symmetry. Here, this ion is considered to vibrate in the $X$ direction. Note again that $x=X, y=Y$, and $z=Z$ are realized in this system, as described in Appendix C.1. When the representative terms in $V_{\mathrm{SA}}$ of eq. (120) and $V_{\mathrm{SF}}$ of eq. (121) (see Figs. 3 - 8) are adopted, the Hamiltonian is written as follows:

$$
\begin{aligned}
& \mathcal{H}_{0}^{\mathrm{AS}}=D S_{Z}^{2}+\hbar \omega a^{\dagger} a, \\
& \mathcal{V}_{\mathrm{SA} 1}=\Lambda_{Z}^{k} S_{Z}\left(-a+a^{\dagger}\right), \\
& \mathcal{V}_{\mathrm{SA} 2}=\left[\Lambda_{X, Z}^{\prime}\left(S_{X} S_{Z}+S_{Z} S_{X}\right)+\Lambda_{Y, Z}^{\prime}\left(S_{Y} S_{Z}+S_{Z} S_{Y}\right)\right]\left(a+a^{\dagger}\right), \\
& \mathcal{V}_{\mathrm{SA} 3}=G\left[D S_{Z}^{2}+F S(S+1)\right]\left(a+a^{\dagger}\right), \\
& \mathcal{V}_{\mathrm{SF}}=\Lambda_{X, Z}^{\mathrm{SF}}\left(S_{X} S_{Z}+S_{Z} S_{X}\right)+\Lambda_{Y, Z}^{\mathrm{SF}}\left(S_{Y} S_{Z}+S_{Z} S_{Y}\right),
\end{aligned}
$$

where $a\left(a^{\dagger}\right)$ is the annihilation (creation) operator of the atomic vibration in the $X$ direction, and $\omega(>0)$ is the angular frequency in this direction. The coefficients $D, F, G$, and $\Lambda_{Z}^{k}$ are eqs. (C·17), (C·19), (C·62), and (C.29), respectively. In addition, $\Lambda_{X, Z}^{\prime}$ and $\Lambda_{Y, Z}^{\prime}$ correspond to eq. (C.76), while $\Lambda_{X, Z}^{\mathrm{SF}}$ and $\Lambda_{Y, Z}^{\mathrm{SF}}$ to eq. (C.54). The state $|u\rangle$ is written as $\left|S_{Z}, n\right\rangle$, where $n$ is the vibrational quantum number.

For this model, we choose $S=2$ (see $\S 4$ ) and $D=-|D|$ using examples from the experimental and theoretical studies of the Fe ion on the CuN surface. ${ }^{10}$ As shown in Fig. 13, the model exhibits bistability between $S_{Z}=-2$ and 2 . The energy difference between the ground states and the first excited states is $3|D|$, while that between the first excited states and the second excited state is $|D|$.

By using eqs. (141) - (146), the respective $W_{u \rightarrow v}^{i}$ 's are obtained as follows:

$$
\begin{aligned}
W_{S_{Z}, n \rightarrow S_{Z}, n-1}^{\mathrm{SA} 1}= & \frac{2 \pi}{\hbar}\left|\Lambda_{Z}^{k}\right|^{2} S_{Z}^{2} n \frac{1}{\pi} \frac{\hbar / \tau}{(\hbar \omega)^{2}+(\hbar / \tau)^{2}}, \\
W_{S_{Z}, n \rightarrow S_{Z}, n+1}^{\mathrm{SA} 1}= & \frac{2 \pi}{\hbar}\left|\Lambda_{Z}^{k}\right|^{2} S_{Z}^{2}(n+1) \frac{1}{\pi} \frac{\hbar / \tau}{(\hbar \omega)^{2}+(\hbar / \tau)^{2}}, \\
W_{S_{Z}, n \rightarrow S_{Z} \pm 1, n-1}^{\mathrm{SA} 2}= & \frac{2 \pi}{\hbar} \frac{\left[\left(\Lambda_{X, Z}^{\prime}\right)^{2}+\left(\Lambda_{Y, Z}^{\prime}\right)^{2}\right]}{4}\left(2 S_{Z} \pm 1\right)^{2}\left(S \mp S_{Z}\right)\left(S \pm S_{Z}+1\right) n \\
& \times \frac{1}{\pi} \frac{\hbar / \tau}{\left[-|D|\left( \pm 2 S_{Z}+1\right)-\hbar \omega\right]^{2}+(\hbar / \tau)^{2}}, \\
W_{S_{Z}, n \rightarrow S_{Z} \pm 1, n+1}^{\mathrm{SA} 2}= & \frac{2 \pi}{\hbar} \frac{\left[\left(\Lambda_{X, Z}^{\prime}\right)^{2}+\left(\Lambda_{Y, Z}^{\prime}\right)^{2}\right]}{4}\left(2 S_{Z} \pm 1\right)^{2}\left(S \mp S_{Z}\right)\left(S \pm S_{Z}+1\right)(n+1) \\
& \times \frac{1}{\pi} \frac{\hbar / \tau}{\left[-|D|\left( \pm 2 S_{Z}+1\right)+\hbar \omega\right]^{2}+(\hbar / \tau)^{2}}, \\
W_{S_{Z}, n \rightarrow S_{Z}, n-1}^{\mathrm{SA} 3}= & \frac{2 \pi}{\hbar} G^{2}\left[-|D| S_{Z}^{2}+F S(S+1)\right]^{2} n \frac{1}{\pi} \frac{\hbar / \tau}{(\hbar \omega)^{2}+(\hbar / \tau)^{2}},
\end{aligned}
$$


J. Phys. Soc. Jpn.

Full PAPER

$$
\begin{aligned}
& W_{S_{Z}, n \rightarrow S_{Z}, n+1}^{\mathrm{SA} 3}=\frac{2 \pi}{\hbar} G^{2}\left[-|D| S_{Z}^{2}+F S(S+1)\right]^{2}(n+1) \frac{1}{\pi} \frac{\hbar / \tau}{(\hbar \omega)^{2}+(\hbar / \tau)^{2}}, \\
& W_{S_{Z}, n \rightarrow S_{Z} \pm 1, n}^{\mathrm{SF}}=\frac{2 \pi}{\hbar} \frac{\left[\left(\Lambda_{X, Z}^{\mathrm{SF}}\right)^{2}+\left(\Lambda_{Y, Z}^{\mathrm{SF}}\right)^{2}\right]}{4}\left(2 S_{Z} \pm 1\right)^{2}\left(S \mp S_{Z}\right)\left(S \pm S_{Z}+1\right) \frac{1}{\pi} \frac{\hbar / \tau}{D^{2}\left( \pm 2 S_{Z}+1\right)^{2}+(\hbar / \tau)^{2}},
\end{aligned}
$$

where $\Lambda_{Z}^{k}$ is an imaginary number, whereas the other coefficients are real number (see $\S 4$ ). The transition probability $W_{S_{Z}, n \rightarrow S_{Z} \pm 1, n-1}^{\mathrm{SA} 2}\left(W_{S_{Z}, n \rightarrow S_{Z} \pm 1, n+1}^{\mathrm{SA} 2}\right)$ corresponds to a probability for a transition with the conservation of energy in the case of $\hbar \omega=-|D|\left( \pm 2 S_{Z}+1\right)\left(\hbar \omega=|D|\left( \pm 2 S_{Z}+\right.\right.$ 1)). The other $W_{u \rightarrow v}^{i}$ 's are probabilities for transitions with the nonconservation of energy, irrespective of $\omega(>0)$.

The parameters are now set to be $|D|=1.5 \quad \mathrm{meV},{ }^{10} \quad \eta=0.05$ (see Appendix ), $\Lambda_{Z}^{k}=-\left(1.00 \times 10^{-5}\right)|D| \mathrm{i}, \quad \Lambda_{X, Z}^{\prime}=\left(7.02 \times 10^{-3}\right)|D|, \quad \Lambda_{Y, Z}^{\prime}=-\left(1.94 \times 10^{-3}\right)|D|, \quad G=9.40 \times 10^{-3}$, $F=-1.25|D|, \Lambda_{X, Z}^{\mathrm{SF}}=\left(7.47 \times 10^{-1}\right)|D|$, and $\Lambda_{Y, Z}^{\mathrm{SF}}=-\left(2.07 \times 10^{-1}\right)|D|$. Here, $\Lambda_{X, Z}^{\prime}, \Lambda_{Y, Z}^{\prime}, G, \Lambda_{X, Z}^{\mathrm{SF}}$, and $\Lambda_{Y, Z}^{\mathrm{SF}}$ have been respectively evaluated from $\Lambda_{X, Z ; x}^{\prime}, \Lambda_{Y, Z ; x}^{\prime}, G_{x}, \Lambda_{X, Z}^{\mathrm{SF}}$, and $\Lambda_{Y, Z}^{\mathrm{SF}}$ at $f=1$ THz in Figs. 5 - 7, while $\Lambda_{Z}^{k}$ has been roughly estimated from $\Lambda_{z, x}^{k}$ at $f=1 \mathrm{THz}$ in the upper panel of Fig. 11. In addition, $F$ has been evaluated from the above $|D|$ and eqs. (C.19) and $(\mathrm{C} \cdot 17)$ with $\Delta_{s}=0.45 \Delta_{m}$.

In Fig. 14, we show the $S_{Z}$ dependence of $W_{u \rightarrow v}^{i}$ in the case of $\hbar \omega=3|D|$. When $\tau=10^{-5} \mathrm{~s}$, $W_{-2, n \rightarrow-1, n-1}^{\mathrm{SA} 2} / n, W_{-1, n \rightarrow-2, n+1}^{\mathrm{SA} 2} /(n+1), W_{1, n \rightarrow 2, n+1}^{\mathrm{SA} 2} /(n+1)$, and $W_{2, n \rightarrow 1, n-1}^{\mathrm{SA} 2} / n$ take the extremely large values because of the transition with the conservation of energy. When $\tau=10^{-10}$ $\mathrm{s}$, however, such probabilities get closer to $W_{S_{Z}, n \rightarrow S_{Z} \pm 1, n}^{\mathrm{SF}}$. For each $\tau$, when a ratio in $W_{u \rightarrow v}^{i}$ is defined as the largest $W_{u \rightarrow v}^{i}$ divided by the smallest one, the ratio of $\tau=10^{-5} \mathrm{~s}$ is larger than that of $\tau=10^{-10} \mathrm{~s}$ because of the sharpness of peaks of $W_{u \rightarrow v}^{i}$. Figure 15 shows the $S_{Z}$ dependence of $W_{u \rightarrow v}^{i}$ in the case of $\hbar \omega=2|D|$. All the $W_{u \rightarrow v}^{i}$ 's are the probabilities for the transitions with the nonconservation of energy. The probability $W_{S_{Z}, n \rightarrow S_{Z} \pm 1, n}^{\mathrm{SF}}$ thus becomes dominant for $\tau=10^{-5}$ and $10^{-10} \mathrm{~s}$, indicating that the magnitudes of the coefficients of $\mathcal{V}_{\mathrm{SF}}$ are larger than those of the other coefficients.

\section{Conclusions}

Bearing in mind a single magnetic ion on a substrate or the ion trapped between a tip and a substrate, we derived a spin-atomic vibration interaction and a spin-flip Hamiltonian of a single atomic spin in a crystal field due to the surrounding ions. Here, the spin-atomic vibration interaction meant the interaction between the spin of the magnetic ion and the vibration of this ion. The spin-flip Hamiltonian represented terms containing spin-flip operators and no atomic vibration operators.

In the derivation, we applied the perturbation theory to the following model: the crystal field potential energy in the equilibrium state was contained in the unperturbed Hamiltonian, 
J. Phys. Soc. Jpn.

Full PaPer
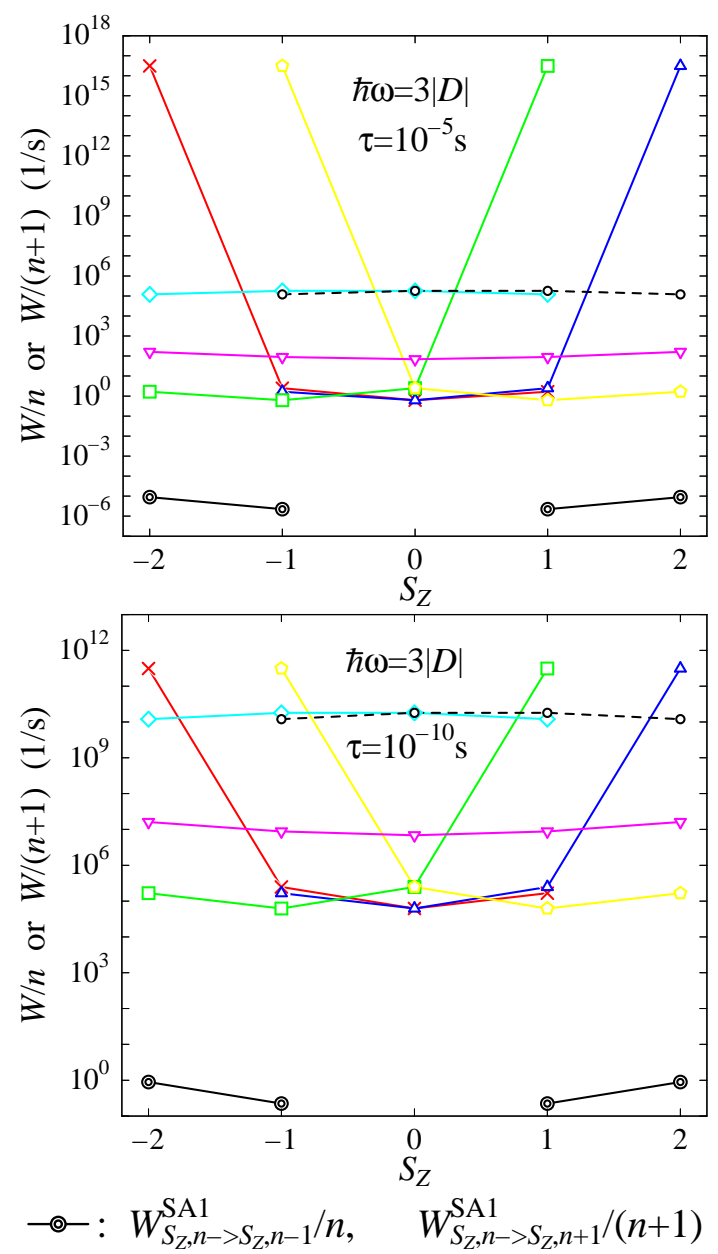

$-\leftarrow: W_{S_{Z}, n->S_{Z}+1, n-1}^{\mathrm{SA} 2} / n$

$\backsim: W_{S_{Z}, n->S_{Z}-1, n-1}^{\mathrm{SA}} / n$

$\rightarrow \square: W_{S_{Z}, n->S_{Z}+1, n+1}^{\mathrm{SA} 2} /(n+1)$

$: W_{S_{Z}, n->S_{Z}-1, n+1}^{\mathrm{SA} 2} /(n+1)$

$\rightarrow-W_{S_{Z}, n->S_{Z}, n-1}^{\mathrm{SA}} / n, \quad W_{S_{Z}, n->S_{Z}, n+1}^{\mathrm{SA}} /(n+1)$

$\prec-W_{S_{Z}, n->S_{Z}+1, n}^{\mathrm{SF}}$

- ०- : $W_{S_{Z}, n->S_{Z}-1, n}^{\mathrm{SF}}$

Fig. 14. $Z$ component of the spin $S_{Z}$ dependences of $W_{S_{Z}, n \rightarrow S_{Z}, n-1}^{\mathrm{SA} 1} / n, W_{S_{Z}, n \rightarrow S_{Z} \pm 1, n-1}^{\mathrm{SA} 2} / n$, $W_{S_{Z}, n \rightarrow S_{Z} \pm 1, n+1}^{\mathrm{SA} 2} /(n+1), W_{S_{Z}, n \rightarrow S_{Z}, n-1}^{\mathrm{SA} 3} / n, W_{S_{Z}, n \rightarrow S_{Z}, n+1}^{\mathrm{SA} 3} /(n+1), W_{S_{Z}, n \rightarrow S_{Z} \pm 1, n}^{\mathrm{SF}}$ of the $S=2$ system with $\hbar \omega=3|D|$. Upper panel: $\tau=10^{-5}$ s. Lower panel: $\tau=10^{-10} \mathrm{~s}$. The energy levels of this system are shown in Fig. 13.

while the spin-orbit interaction and the modulation of crystal field potential energy due to the vibration displacement were the perturbed terms. The model also took into account the difference in vibration displacement between the effective nucleus and the electrons, $\left|\eta \Delta r_{\mathrm{n}}\right|$, where $\Delta r_{\mathrm{n}}$ is the displacement of the nucleus, and $\eta(0 \leq \eta<1)$ is a dimensionless quantity 

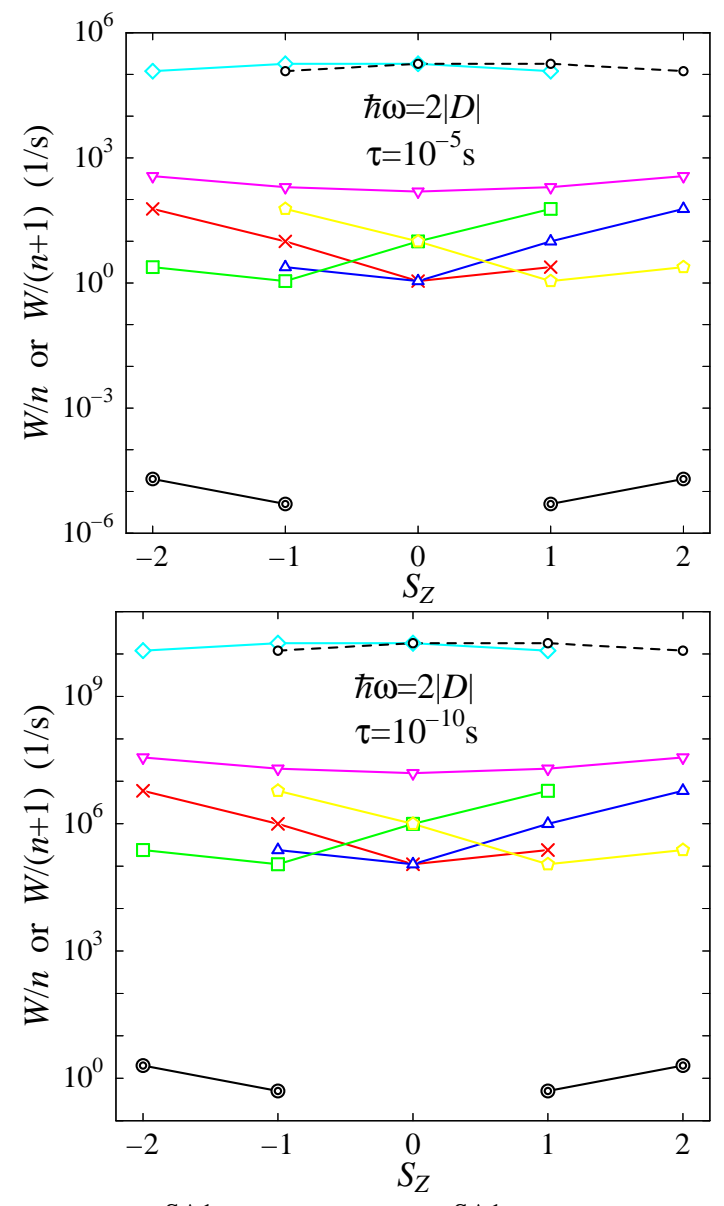

-๑ : $W_{S_{Z}, n->S_{Z}, n-1}^{\mathrm{SA} 1} / n, \quad W_{S_{Z}, n->S_{Z}, n+1}^{\mathrm{SA} 1} /(n+1)$

$\rightarrow \leftarrow: W_{S_{Z}, n->S_{Z}+1, n-1}^{\mathrm{SA} 2} / n$

$\neg-W_{S_{Z}, n->S_{Z}-1, n-1}^{\mathrm{SA} 2} / n$

$-\square-: W_{S_{Z}, n->S_{Z}+1, n+1}^{\mathrm{SA} 2} /(n+1)$

: $W_{S_{Z}, n->S_{Z}-1, n+1}^{\mathrm{SA} 2} /(n+1)$

$\rightarrow-: W_{S_{Z}, n->S_{Z}, n-1}^{\mathrm{SA}} / n, \quad W_{S_{Z}, n->S_{Z}, n+1}^{\mathrm{SA}} /(n+1)$

$\diamond: W_{S_{Z}, n->S_{Z}+1, n}^{\mathrm{SF}}$

-о- : $W_{S_{Z}, n->S_{Z}-1, n}^{\mathrm{SF}}$

Fig. 15. $Z$ component of the spin $S_{Z}$ dependences of $W_{S_{Z}, n \rightarrow S_{Z}, n-1}^{\mathrm{SA} 1} / n, W_{S_{Z}, n \rightarrow S_{Z} \pm 1, n-1}^{\mathrm{SA} 2} / n$, $W_{S_{Z}, n \rightarrow S_{Z} \pm 1, n+1}^{\mathrm{SA} 2} /(n+1), W_{S_{Z}, n \rightarrow S_{Z}, n-1}^{\mathrm{SA} 3} / n, W_{S_{Z}, n \rightarrow S_{Z}, n+1}^{\mathrm{SA} 3} /(n+1), W_{S_{Z}, n \rightarrow S_{Z} \pm 1, n}^{\mathrm{SF}}$ of the $S=2$ system with $\hbar \omega=2|D|$. Upper panel: $\tau=10^{-5}$ s. Lower panel: $\tau=10^{-10}$ s. The energy levels of this system are shown in Fig. 13.

characterizing the difference. The unperturbed wave function $\psi_{\zeta}(\zeta=1-5)$ was roughly set to be $\psi_{\zeta}=d_{\zeta}+c_{d} \sum_{m=1(\neq \zeta)}^{5} \overline{d_{m}}+c_{p} \sum_{n=1}^{3} \overline{p_{n}}$. Here, $d_{\zeta}$ was the dominant 3 d orbital, while $\overline{d_{m}}$ and $\overline{p_{n}}$ were the other $3 \mathrm{~d}$ orbital and $4 \mathrm{p}$ orbital in the same magnetic ion, respectively. The coefficient for the d orbital, $c_{d}$, (the coefficient for $\mathrm{p}$ orbital, $c_{p}$,) represented the degree of d-d 
hybridization (d-p hybridization).

As results, the spin-atomic vibration interaction was obtained as $\sum_{I} c_{1, I} S_{I}(-a+$ $\left.a^{\dagger}\right)+\sum_{I, J}\left(c_{2, I, J} S_{I} S_{J} a+c_{3, I, J} S_{I} S_{J} a^{\dagger}\right)$, and the spin-flip Hamiltonian was expressed as $\sum_{I, J} c_{4, I, J} S_{I} S_{J}$, with $I, J=X, Y, Z$. Here, $S_{I}$ was the $I$ component of the spin $\boldsymbol{S}=\left(S_{X}, S_{Y}, S_{Z}\right)$, and $a\left(a^{\dagger}\right)$ was the annihilation operator (creation operator) of the vibration of the magnetic ion. The coefficients of the spin-atomic vibration interaction $c_{1, I}, c_{2, I, J}$, and $c_{3, I, J}$ contained $\eta, c_{p}, c_{d}$, and $f$, while that of the spin-flip Hamiltonian $c_{4, I, J}$ had $c_{d}$, where $f$ was a vibration frequency.

The respective details about the spin-atomic vibration interaction, spin-flip Hamiltonian, and transition probability per unit time are written as follows:

(1) Spin-atomic vibration interaction

a. The spin-atomic vibration interaction appeared owing to the difference in vibration displacement and the d-p hybridization. Concretely speaking, this interaction was proportional to $\eta$ and also present for $c_{p} \neq 0$. The condition of $c_{p} \neq 0$ was attributed to the result that the coefficients of this interaction contained the similar formula to the matrix element of the electric dipole transition.

b. Most of the coefficients of the $S_{I} S_{J} a_{\xi}$ and $S_{I} S_{J} a_{\xi}^{\dagger}$ terms (i.e., $c_{2, I, J}$ and $c_{3, I, J}$ ) showed the $1 / \sqrt{f}$ dependence, indicating that these coefficients were proportional to the vibration displacement of the nucleus, $\Delta \boldsymbol{r}_{n}(\propto 1 / \sqrt{f})$. In contrast, the coefficient of the $S_{I}\left(-a_{\xi}+a_{\xi}^{\dagger}\right)$ term (i.e., $\left.c_{1, I}\right)$ had the $\sqrt{f}$ dependence because it was proportional to the momentum of the nucleus, $\Delta \boldsymbol{p}_{n}(\propto \sqrt{f})$. Note that the coefficient of the conventional spin-phonon interaction was proportional to $\sqrt{f}$, where this spin-phonon interaction meant an interaction between a single spin and the phonon of a lattice system,

c. In the case of the Fe ion in the crystal field of tetragonal symmetry, the magnitudes of the coefficients of the spin-atomic vibration interaction became larger than that of the spin-phonon interaction by Mattuck and Strandberg in the specific region of $f$. The main reason for this was that the mass of the oscillator of the spin-atomic vibration interaction was smaller than that of the spin-phonon interaction. Here, the oscillator of the spin-atomic vibration interaction was the single magnetic ion, while that of the spin-phonon interaction consisted of all the ions in the unit cell. Note that the decrease in the mass of the oscillator increased the magnitude of vibration displacement, and furthermore the increase in the magnitude of the displacement enhanced the magnitude of the dominant term of the spin-atomic vibration interaction.

(2) Spin-flip Hamiltonian

a. The spin-flip Hamiltonian stemmed from only the d-d hybridization, and was independent of the atomic vibration. In other words, the spin-flip Hamiltonian existed only for $c_{d} \neq 0$, and did not contain $f$ and $\eta$. 
J. Phys. Soc. Jpn.

Full PAPER

b. In the case of the Fe ion in the crystal field of tetragonal symmetry, the magnitudes of the coefficients of the spin-flip Hamiltonian tended to be larger than those of the spin-atomic vibration interaction. This tendency was attributed to the result that the coefficients of the spin-flip Hamiltonian did not contain $\eta$, whereas those of the spinatomic vibration interaction were proportional to $\eta$, where $\eta=0.05$ was set in this paper.

(3) Transition probability per unit time

The transition probability per unit time was investigated for a simple model of the $\mathrm{Fe}$ ion. The unperturbed Hamiltonian was $-|D| S_{Z}^{2}+\hbar \omega a^{\dagger} a$, with $S=2$, where $D$ was an anisotropy constant, and $\omega(=2 \pi f)$ was the angular frequency. As to $-|D| S_{Z}^{2}$, the energy difference between the ground states and the first excited states was $3|D|$, while that between the first excited states and the second excited state was $|D|$. The perturbed Hamiltonians were composed of the representative terms in the spin-atomic vibration interaction and spin-flip Hamiltonian. Note here that the magnitudes of the coefficients of the spin-atomic vibration interaction were set to be smaller than those of the spin-flip Hamiltonian (see $2 \mathrm{~b}$ ). The transition probability due to the spin-atomic vibration interaction (e.g., $S_{X} S_{Z}\left(a+a^{\dagger}\right)$ ) corresponded to the probability for the transition with the conservation of energy, depending on $\hbar \omega$ and the initial spin state (i.e., $S_{Z}$ ). In contrast, the transition probability due to the spin-flip Hamiltonian (e.g., $S_{X} S_{Z}$ ) became the probability for the transition with the nonconservation of energy. In the cases of $\hbar \omega=3|D|$ and specific $S_{Z}$ 's, the transition probability due to the spin-atomic vibration interaction took the largest value in all the probabilities because of the transition with the conservation of energy. In the case of $\hbar \omega=2|D|$, all the transition probabilities were the probabilities for the transitions with the nonconservation of energy. The transition probability due to the spin-flip Hamiltonian thus became dominant, indicating that the magnitudes of the coefficients of the spin-flip Hamiltonian were larger than those of the other coefficients.

\section{Acknowledgments}

We would like to thank Prof. Toshiharu Hoshino of Shizuoka University for useful discussion. This work has been supported by a Grant-in-Aid for Young Scientists (B) (No. 20710076) from the Japan Society for the Promotion of Science.

\section{Appendix A: Difference in Displacement between Nucleus and Electron}

Let us consider a model in which a single electron is present in the $\sigma$-spin shell (e.g., $\mathrm{Fe}^{2+}$ : an electron exists in the down-spin shell). For this model, we evaluate the difference in vibration displacement between an effective nucleus and the electron in the $\sigma$-spin shell. The effective nucleus consists of a nucleus and core electrons, where the core electrons correspond to the electrons other than those in the $\sigma$-spin shell. We here use the theory of electronic polarizability in ref. 53 . 


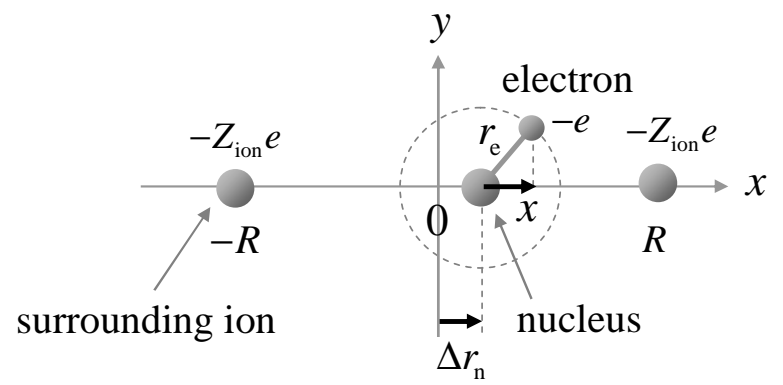

Fig. A.1. Schematic illustration of the vibrating magnetic ion. The effective nucleus has the displacement $\Delta r_{\mathrm{n}}$ in the $x$ direction. The electron then moves in a circular orbit around the nucleus fixed at $\Delta r_{\mathrm{n}}$, and simultaneously experiences the crystalline electric field due to the surrounding ions. The $x$ component of the position of the electron is written as $\Delta r_{\mathrm{n}}+x$.

We consider a situation in which only the magnetic ion vibrates in the $x$ direction, while the surrounding ions are rigid. Such a situation is schematically illustrated in Fig. A.1. In particular, this ion acts as a harmonic oscillator in the vicinity of a stable equilibrium position. This position corresponds to a local minimum point of a potential energy of the system. Here, the energy consists of "a Coulomb potential energy between the magnetic ion and the surrounding ions" and "a repulsive potential energy due to the Pauli exclusion principle and the overlap of electrons between the magnetic ion and the surrounding ions". 54 Now, the magnetic ion is regarded as a mass point with the mass of this ion, $M$. The position of the mass point corresponds to the gravity point of the magnetic ion, which may be, in other words, the position of the effective nucleus. When this mass point has the displacement from the equilibrium position, $\Delta r_{\mathrm{n}}$, the equation of motion of the mass point is written as

$$
M \Delta \ddot{r}_{\mathrm{n}}=-M \omega_{\mathrm{n}}^{2} \Delta r_{\mathrm{n}},
$$

where $\omega_{\mathrm{n}}$ is the vibration frequency of the magnetic ion.

When the effective nucleus is located at $\Delta r_{\mathrm{n}}$, the electron is considered to move in a circular orbit around "the nucleus fixed at $\Delta r_{\mathrm{n}}$ ". In particular, we focus on the $x$ component of the circular motion of the electron in the $x y$ plane (see Fig. A.1). The $x$ component of the position of the electron is written by $\Delta r_{\mathrm{n}}+x$. Here, $x\left(-r_{\mathrm{e}} \leq x \leq r_{\mathrm{e}}\right)$ is the $x$ component of a vector that points from the nucleus to the electron, with $r_{\mathrm{e}}$ being the electron radius. In addition, the electron experiences the crystalline electric field due to the surrounding ions. For this electron, we have the following equation of motion in the $x$ direction:

$$
m\left(\Delta \ddot{r}_{\mathrm{n}}+\ddot{x}\right)=-m \omega_{\mathrm{e}}^{2} x-e E_{\mathrm{ion}},
$$

where $\omega_{\mathrm{e}}$ is the angular velocity of the circular motion of the electron, $-e(<0)$ is the electronic charge, and $E_{\text {ion }}$ is the $x$ component of the crystalline electric field.

On the left-hand side of eq. (A.2), we note that $\left|\Delta \ddot{r}_{n}\right|\left(=\left|\omega_{n}^{2} \Delta r_{n}\right|\right)$ is estimated to be much 
J. Phys. Soc. Jpn.

Full PAPER

smaller than $|\ddot{x}|\left(\sim\left|\omega_{\mathrm{e}}^{2} x\right|\right)$, because of $10^{-12} \lesssim\left(\omega_{\mathrm{n}} / \omega_{\mathrm{e}}\right)^{2} \lesssim 10^{-4}$ and $9.47 \times 10^{-3} \lesssim\left|\Delta r_{\mathrm{n}} / x\right| \lesssim$ $9.47 \times 10^{-1}$. We here use $\omega_{\mathrm{n}}=2 \pi f$ with $0.01 \mathrm{THz} \leq f \leq 100 \mathrm{THz}$, and $\omega_{\mathrm{e}}$ of eq. (A.6). The magnitude of the displacement $\left|\Delta r_{\mathrm{n}}\right|$ has been considered to be $9.47 \times 10^{-4} \mathrm{~nm} \lesssim \sqrt{\overline{\Delta r_{\mathrm{n}}^{2}}} \lesssim$ $9.47 \times 10^{-2} \mathrm{~nm}$ in Appendix . In addition, $|x|$ has been roughly replaced with the square root of the time average of $x^{2}$, i.e., $r_{\mathrm{e}} / \sqrt{2}(\sim 0.1 \mathrm{~nm})$, where $r_{\mathrm{e}}$ corresponds to $r_{n}$ of eq. (A.9).

Thus, by neglecting $\Delta \ddot{r}_{\mathrm{n}}$, eq. $(\mathrm{A} \cdot 2)$ is rewritten as

$$
m \ddot{x}=-m \omega_{\mathrm{e}}^{2} x-e E_{\mathrm{ion}} .
$$

Regarding $E_{\text {ion }}$, we consider a case in which the surrounding ions with the minus charge $-Z_{\text {ion }} e(<0)$ are respectively located at $-R$ and $R$ in the $x$ axis as the point charges. Under the conditions of $r_{\mathrm{e}} / R \ll 1,|x| / R \ll 1$, and $\left|\Delta r_{\mathrm{n}}\right| / R \ll 1, E_{\text {ion }}$ is obtained as

$$
\begin{aligned}
E_{\mathrm{ion}} & =\frac{-Z_{\mathrm{ion}} e}{4 \pi \epsilon_{0}}\left\{\frac{-\left(R-\Delta r_{\mathrm{n}}-x\right)}{\left[\left(R-\Delta r_{\mathrm{n}}-x\right)^{2}+r_{\mathrm{e}}^{2}-x^{2}\right]^{3 / 2}}+\frac{R+\Delta r_{\mathrm{n}}+x}{\left[\left(R+\Delta r_{\mathrm{n}}+x\right)^{2}+r_{\mathrm{e}}^{2}-x^{2}\right]^{3 / 2}}\right\} \\
& \approx \frac{Z_{\mathrm{ion}} e}{\pi \epsilon_{0} R^{3}}\left(x+\Delta r_{\mathrm{n}}\right),
\end{aligned}
$$

where terms higher than the first order of $r_{\mathrm{e}} / R, x / R$, and $\Delta r_{\mathrm{n}} / R$ have been neglected. Note that, in eq. (A.4), the effect of the repulsive potential energy due to the overlap of electrons has not been taken into account for simplicity. Substituting eq. (A.4) into eq. (A.3), we obtain

$$
m \ddot{x}=-\left(m \omega_{\mathrm{e}}^{2}+\frac{Z_{\text {ion }} e^{2}}{\pi \epsilon_{0} R^{3}}\right) x-\frac{Z_{\text {ion }} e^{2}}{\pi \epsilon_{0} R^{3}} \Delta r_{\mathrm{n}} .
$$

Here, by using the speed of electron, $v=r_{\mathrm{e}} \omega_{\mathrm{e}}$, the expression for the equilibrium of force, $m v^{2} / r_{\mathrm{e}}=Z_{\mathrm{eff}} e^{2} /\left(4 \pi \epsilon_{0} r_{\mathrm{e}}^{2}\right)$, and the Bohr's quantization condition $r_{\mathrm{e}} m v=n \hbar$ with $n=1,2,3, \cdots$, $\omega_{\mathrm{e}}$ is expressed as

$$
\omega_{\mathrm{e}}=\frac{Z_{\mathrm{eff}}^{2} h}{2 \pi m n^{3} a_{\mathrm{B}}^{2}},
$$

with $a_{\mathrm{B}}=\epsilon_{0} h^{2} /\left(\pi m e^{2}\right)$, where $a_{\mathrm{B}}$ is the Bohr radius.

The center position of the oscillation of the electron, $x_{c}$, corresponds to the difference in vibration displacement between the nucleus and the electron. This $x_{c}$ is obtained by putting $\ddot{x}=0$ and $x=x_{c}$ in eq. $(\mathrm{A} \cdot 5)$ :

$$
\begin{aligned}
& x_{c}=-\eta \Delta r_{\mathrm{n}}, \\
& \eta=\frac{4\left(Z_{\text {ion }} / Z_{\text {eff }}\right)\left(r_{n} / R\right)^{3}}{1+4\left(Z_{\text {ion }} / Z_{\text {eff }}\right)\left(r_{n} / R\right)^{3}}, \\
& r_{n}=n^{2} a_{\mathrm{B}} / Z_{\text {eff }},
\end{aligned}
$$

with $0 \leq \eta<1$. Here, $x_{c}=0$ is confirmed for $Z_{\text {ion }}=0$.

In Fig. A.2, we show the $Z_{\text {ion }} / Z_{\text {eff }}$ dependence of $\eta$ for $R=2.5 r_{n}, 3 r_{n}, 4 r_{n}$, and $5 r_{n}$. The quantity $\eta$ increases with increasing $Z_{\text {ion }} / Z_{\text {eff }}$. In the case of $Z_{\text {ion }} / Z_{\text {eff }}=0.2$, we obtain $\eta=0.05$ for $R=2.5 r_{n}, \eta=0.03$ for $R=3 r_{n}, \eta=0.01$ for $R=4 r_{n}$, and $\eta=0.006$ for $R=5 r_{n}$. 
As an application, we consider $\eta$ of the single Fe ion on the CuN surface. ${ }^{10}$ The effective nuclear charge of the $3 \mathrm{~d}$ orbital of $\mathrm{Fe}, Z_{\text {eff }}$, is obtained as $Z_{\text {eff }}=6.25$ according to Slater's rules, ${ }^{37}$ while $Z_{\text {ion }}$ arising from the nearest-neighbor $\mathrm{N}$ is set to be $Z_{\text {ion }}=1.4$ on the basis of ref. 10. From $Z_{\text {ion }} / Z_{\text {eff }}=0.224$, the largest $\eta$ is evaluated to be about 0.05 .

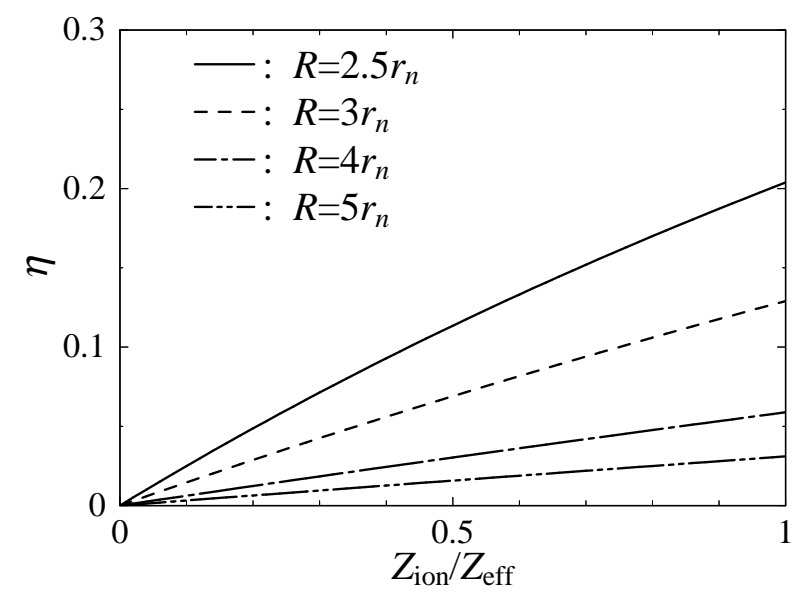

Fig. A.2. Quantity $Z_{\text {ion }} / Z_{\text {eff }}$ dependence of $\eta$ for $R=2.5 r_{n}, 3 r_{n}, 4 r_{n}$, and $5 r_{n}$.

\section{Appendix B: Magnitude of Displacement of Nucleus $\left|\Delta r_{\mathrm{n}}\right|$}

On the basis of eq. (A-1), we evaluate the magnitude of vibration displacement of the effective nucleus, $\left|\Delta r_{\mathrm{n}}\right|$. Here, $\left|\Delta r_{\mathrm{n}}\right|$ is defined as the square root of the time average of $\Delta r_{\mathrm{n}}^{2}$.

The solution of eq. (A.1) is first obtained as

$$
\Delta r_{\mathrm{n}}=A \cos \left(\omega_{\mathrm{n}} t\right)+B \sin \left(\omega_{\mathrm{n}} t\right)
$$

where $A$ and $B$ are coefficients. The total energy is then written as

$$
\begin{aligned}
E & =\frac{1}{2} M \Delta \dot{r}_{\mathrm{n}}^{2}+\frac{1}{2} M \omega_{\mathrm{n}}^{2} \Delta r_{\mathrm{n}}^{2} \\
& =\frac{1}{2} M \omega_{\mathrm{n}}^{2}\left(A^{2}+B^{2}\right) \\
& =M \omega_{\mathrm{n}}^{2} \overline{\Delta r_{\mathrm{n}}^{2}} .
\end{aligned}
$$

Here, $\overline{\Delta r_{\mathrm{n}}^{2}}$ is the time average of $\Delta r_{\mathrm{n}}^{2}$, given by

$$
\overline{\Delta r_{\mathrm{n}}^{2}}=\lim _{t^{\prime} \rightarrow \infty} \frac{1}{t^{\prime}} \int_{0}^{t^{\prime}} \Delta r_{\mathrm{n}}^{2} \mathrm{~d} t=\frac{1}{2}\left(A^{2}+B^{2}\right) .
$$

On the other hand, the energy of a quantum harmonic oscillator is expressed as $(\langle n\rangle+$ $1 / 2) \hbar \omega_{\mathrm{n}}$. Here, $\langle n\rangle$ is the thermal average of the vibrational quantum number, given by $\langle n\rangle$ $=\left[\exp \left(\hbar \omega_{\mathrm{n}} / k_{\mathrm{B}} T\right)-1\right]^{-1}$, where $T$ is the temperature and $k_{\mathrm{B}}$ is the Boltzmann constant. 
We now equate $E$ of eq. (B.2) with the above energy; ${ }^{55}$ that is,

$$
M \omega_{\mathrm{n}}^{2} \overline{\Delta r_{\mathrm{n}}^{2}}=\left(\langle n\rangle+\frac{1}{2}\right) \hbar \omega_{\mathrm{n}}
$$

From eq. (B.4), we can obtain $\sqrt{\overline{\Delta r_{\mathrm{n}}^{2}}}$. At $T=0 \mathrm{~K}, \sqrt{\overline{\Delta r_{\mathrm{n}}^{2}}}$ is written as

$$
\sqrt{\overline{\Delta r_{\mathrm{n}}^{2}}}=\sqrt{\frac{\hbar}{2 M \omega_{\mathrm{n}}}}
$$

The quantity $\sqrt{\overline{\Delta r_{\mathrm{n}}^{2}}}$ decreases with increasing $M$ and $\omega_{\mathrm{n}}$.

We also rewrite eq. (A.1) as $M \Delta \ddot{r}_{\mathrm{n}}=-C \Delta r_{\mathrm{n}}$, with $C \equiv M \omega_{\mathrm{n}}^{2}$. Here, $C$ is a force constant that corresponds to the second derivative of the interatomic potential with respect to the displacement of the magnetic ion. ${ }^{56}$ Substituting $\omega_{\mathrm{n}}=\sqrt{C / M}$ into eq. (B.5), we obtain

$$
\sqrt{\overline{\Delta r_{\mathrm{n}}^{2}}}=\sqrt{\frac{\hbar}{2 \sqrt{M C}}} .
$$

The quantity $\sqrt{\overline{\Delta r_{\mathrm{n}}^{2}}}$ decreases with increasing $M$ and $C$.

As an application, we consider $\sqrt{\overline{\Delta r_{\mathrm{n}}^{2}}}$ at $T=0 \mathrm{~K}$ for $\mathrm{Fe}^{2+}$, where $M$ of $\mathrm{Fe}^{2+}$ is $M \approx(26+30) \times\left(1.67 \times 10^{-27}\right) \mathrm{kg}$. By using eq. (B.5), $\sqrt{\overline{\Delta r_{\mathrm{n}}^{2}}}$ is evaluated to be $9.47 \times 10^{-4} \mathrm{~nm}$ $\lesssim \sqrt{\overline{\Delta r_{\mathrm{n}}^{2}}} \lesssim 9.47 \times 10^{-2} \mathrm{~nm}$ for $0.01 \mathrm{THz} \leq f \leq 100 \mathrm{THz}$, where $\omega_{\mathrm{n}} \equiv 2 \pi f$.

\section{Appendix C: Expression of Coefficients for Fe Ion}

We describe the expressions of coefficients of the spin-atomic vibration interaction and the spin-flip Hamiltonian of the Fe ion $\left(\mathrm{Fe}^{2+}\right)$ in the crystal field of tetragonal symmetry. For convenience, the coefficients are represented by the following dimensionless quantities: $\Lambda_{\mu, \xi}^{(1)} / \lambda$, $\Lambda_{\mu, \xi}^{k} / \lambda, G_{\xi}, F / D, \Lambda_{I, J ; \xi}^{\prime} / D, \Lambda_{\mu ; \nu, \xi}^{ \pm} / D, \Lambda_{\mu, \xi ; \nu}^{ \pm} / D$, and $\Lambda_{I, J}^{\mathrm{SF}} / D$.

We here focus on one down-spin electron as described in $\S 4$. The energy levels are assumed to be $E_{j}$ of Fig. 2. In addition, we use the notation of $\left.\left.\left.\left.\left.\left.\mid \Psi_{1}\right)=\mid x y\right), \mid \Psi_{2}\right)=\mid y z\right), \mid \Psi_{3}\right)=\mid x z\right)$, $\left.\left.\left.\left|\Psi_{4}\right\rangle=\mid x^{2}-y^{2}\right), \mid \Psi_{5}\right)=\mid 3 z^{2}-r^{2}\right),\left|d_{1}\right\rangle=|x y\rangle,\left|d_{2}\right\rangle=|x z\rangle,\left|d_{3}\right\rangle=|y z\rangle,\left|d_{4}\right\rangle=\left|x^{2}-y^{2}\right\rangle,\left|d_{5}\right\rangle=\left|3 z^{2}-r^{2}\right\rangle$, $\left|p_{1}\right\rangle=|x\rangle,\left|p_{2}\right\rangle=|y\rangle$, and $\left|p_{3}\right\rangle=|z\rangle$. As to $E_{j}, e_{j}, c_{d_{m}}^{(j)}, c_{p_{n}}^{(j)}$, and $C_{j}$, we have the notation and parameter setup in $\S 4.2$.

In the below-mentioned calculations, we will use the following matrix elements:

$$
\begin{aligned}
& \left\langle x z\left|L_{x}\right| x y\right\rangle=\mathrm{i}, \\
& \left\langle x^{2}-y^{2}\left|L_{x}\right| y z\right\rangle=\mathrm{i}, \\
& \left\langle 3 z^{2}-r^{2}\left|L_{x}\right| y z\right\rangle=\sqrt{3} \mathrm{i}, \\
& \left\langle y z\left|L_{y}\right| x y\right\rangle=-\mathrm{i}, \\
& \left\langle x^{2}-y^{2}\left|L_{y}\right| x z\right\rangle=\mathrm{i}, \\
& \left\langle 3 z^{2}-r^{2}\left|L_{y}\right| x z\right\rangle=-\sqrt{3} \mathrm{i},
\end{aligned}
$$


J. Phys. Soc. Jpn.

Full PAPER

$$
\begin{aligned}
& \left\langle x^{2}-y^{2}\left|L_{z}\right| x y\right\rangle=-2 \mathrm{i}, \\
& \left\langle x z\left|L_{z}\right| y z\right\rangle=-\mathrm{i}, \\
& \left\langle z\left|L_{x}\right| y\right\rangle=\mathrm{i}, \\
& \left\langle x\left|L_{y}\right| z\right\rangle=\mathrm{i}, \\
& \left\langle y\left|L_{z}\right| x\right\rangle=\mathrm{i},
\end{aligned}
$$

with $\left\langle d_{j^{\prime}}|\boldsymbol{L}| d_{j}\right\rangle=-\left\langle d_{j}|\boldsymbol{L}| d_{j^{\prime}}\right\rangle$ and $\left\langle p_{j^{\prime}}|\boldsymbol{L}| p_{j}\right\rangle=-\left\langle p_{j}|\boldsymbol{L}| p_{j^{\prime}}\right\rangle \cdot{ }^{42}$

\section{C.1 Principal axis coordinate system}

When an electron in the crystal field of tetragonal symmetry has $E_{j}$ in Fig. 2, we find that $X, Y$, and $Z$ are written as $X=x, Y=y$, and $Z=z$, respectively.

Using eqs. (C.1) - (C.8), we obtain $\mathcal{L}_{\mu, \nu}^{(0)}$ of eq. (90) as

$$
\mathcal{L}^{(0)}=\left(\begin{array}{ccc}
\mathcal{L}_{x, x}^{(0)} & 0 & 0 \\
0 & \mathcal{L}_{y, y}^{(0)} & 0 \\
0 & 0 & \mathcal{L}_{z, z}^{(0)}
\end{array}\right),
$$

where

$$
\begin{aligned}
& \mathcal{L}_{x, x}^{(0)}=C^{4} \frac{\left\langle x y\left|L_{x}\right| x z\right\rangle\left\langle x z\left|L_{x}\right| x y\right\rangle}{e_{x y}-e_{x z}}=-\frac{C^{4}}{\Delta_{s}} \\
& \mathcal{L}_{y, y}^{(0)}=C^{4} \frac{\left\langle x y\left|L_{y}\right| y z\right\rangle\left\langle y z\left|L_{y}\right| x y\right\rangle}{e_{x y}-e_{y z}}=-\frac{C^{4}}{\Delta_{s}} \\
& \mathcal{L}_{z, z}^{(0)}=C^{4} \frac{\left\langle x y\left|L_{z}\right| x^{2}-y^{2}\right\rangle\left\langle x^{2}-y^{2}\left|L_{z}\right| x y\right\rangle}{e_{x y}-e_{x^{2}-y^{2}}}=-\frac{4 C^{4}}{\Delta_{m}} \\
& C=\left(1+4 c_{d}^{2}+3 c_{p}^{2}\right)^{-1 / 2}
\end{aligned}
$$

with $\mathcal{L}_{\mu, \nu}^{(0)}=0$ for $\mu \neq \nu$. Here, we have roughly set $e_{1}-e_{j}=E_{1}-E_{j}$, taking into account $\mid \Delta e_{1}-$ $\Delta e_{j}|\ll| e_{1}-e_{j} \mid$ for $j \neq 1$ (see eq. (45)).

Since $\mathcal{L}^{(0)}$ is a diagonal matrix, we obtain $\theta_{X, x}=\theta_{Y, y}=\theta_{Z, z}=0$ in eqs. (94) and (95), and the other $\theta_{I, \mu}$ 's are $\pi / 2$. Namely, $X, Y$, and $Z$ become $X=x, Y=y$, and $Z=z$, respectively.

\section{C.2 D, E, and F}

Using eqs. (103) - (105) and (C.13) - (C.15), we obtain $D, E$, and $F$ as

$$
\begin{aligned}
& D=\lambda^{2}\left(\mathcal{L}_{Z, Z}^{(0)}-\frac{\mathcal{L}_{X, X}^{(0)}+\mathcal{L}_{Y, Y}^{(0)}}{2}\right)=\lambda^{2} \frac{C^{4}}{\Delta_{m}}\left(\frac{\Delta_{m}}{\Delta_{s}}-4\right), \\
& E=\lambda^{2} \frac{\mathcal{L}_{X, X}^{(0)}-\mathcal{L}_{Y, Y}^{(0)}}{2}=0, \\
& F=\lambda^{2} \frac{\mathcal{L}_{X, X}^{(0)}+\mathcal{L}_{Y, Y}^{(0)}}{2}=-\lambda^{2} \frac{C^{4}}{\Delta_{s}},
\end{aligned}
$$


J. Phys. Soc. Jpn.

Full PAPER

with $\mathcal{L}_{X, X}^{(0)}=\mathcal{L}_{x, x}^{(0)}, \mathcal{L}_{Y, Y}^{(0)}=\mathcal{L}_{y, y}^{(0)}$, and $\mathcal{L}_{Z, Z}^{(0)}=\mathcal{L}_{z, z}^{(0)}$. The bistability (i.e., $D<0$ ) is obtained for $\Delta_{m} / \Delta_{s}<4$. The coefficient $D$ comes close to $-3 \lambda^{2} C^{4} / \Delta_{m}$ as $\Delta_{m} / \Delta_{s}$ comes to 1 . In addition, we find that the conditions of $\Delta_{s} \sim \Delta_{m}$ and a small $\Delta_{m}$ as well as a large $|\lambda|$ enhance $|D|$. The coefficient $E$ becomes 0 owing to $\mathcal{L}_{X, X}^{(0)}=\mathcal{L}_{Y, Y}^{(0)}$.

C.3 $\Lambda_{\mu, \xi}^{(1)} / \lambda$

Using eqs. (55), (7), and $\left.\mid \Psi_{1}\right)=\mid x y$ ), we have

$$
\frac{\Lambda_{\mu, \xi}^{(1)}}{\lambda}=-\eta \frac{1}{\hbar} \mathrm{i} \frac{m}{M} \sqrt{\frac{M \hbar \omega_{\xi}}{2}}(x y|\mu| x y),
$$

with $\mu, \xi=x, y, z$. By using $\mid x y)$ in eq. (122), $(x y|\mu| x y)$ is obtained as follows:

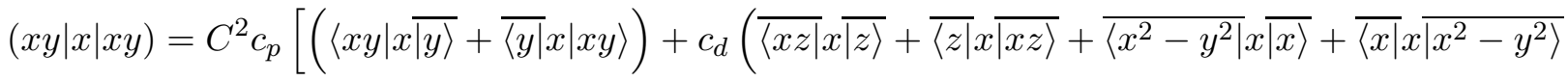

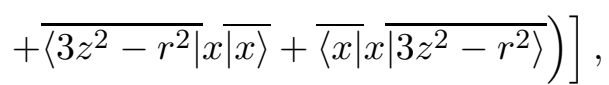

$$
\begin{aligned}
& (x y|y| x y)=C^{2} c_{p}\left[(\langle x y|y| \overline{|x\rangle}+\overline{\langle x|} y \mid x y\rangle)+c_{d}\left(\overline{\langle y z|y| z\rangle}+\overline{\langle z|y| y z\rangle}+\overline{\left\langle x^{2}-y^{2}|y| y\right\rangle}+\overline{\langle y|} y \overline{\left|x^{2}-y^{2}\right\rangle}\right.\right. \\
& \left.\left.+\overline{\left\langle 3 z^{2}-r^{2}|y| y\right\rangle}+\overline{\langle y|y|} \overline{\left.3 z^{2}-r^{2}\right\rangle}\right)\right], \\
& (x y|z| x y)=C^{2} c_{d} c_{p}\left(\overline{\langle x z|} z \overline{|x\rangle}+\overline{\langle x|} z \overline{|x z\rangle}+\overline{\langle y z|} z \overline{|y\rangle}+\overline{\langle y|} z\left|\overline{|y z\rangle}+\overline{\left\langle 3 z^{2}-r^{2}|z| z\right\rangle}+\overline{\langle z|} z\right| \overline{\left.3 z^{2}-r^{2}\right\rangle}\right),
\end{aligned}
$$

where $(x y|\mu| x y)$ vanishes at $c_{p}=0$. The matrix element $\left\langle p_{j^{\prime}}|\mu| d_{j}\right\rangle$ is obtained using eqs. (125) $-(132)$ :

$$
\begin{aligned}
& \langle x|y| x y\rangle=-8.31 \times 10^{-12}, \\
& \left\langle x|x| 3 z^{2}-r^{2}\right\rangle=4.80 \times 10^{-12}, \\
& \left\langle z|z| 3 z^{2}-r^{2}\right\rangle=-9.59 \times 10^{-12}, \\
& \left\langle x|x| x^{2}-y^{2}\right\rangle=-8.31 \times 10^{-12}, \\
& \left\langle y|y| x^{2}-y^{2}\right\rangle=8.31 \times 10^{-12}
\end{aligned}
$$

where $\left\langle d_{j}|\mu| p_{j^{\prime}}\right\rangle=\left\langle p_{j^{\prime}}|\mu| d_{j}\right\rangle$.

C.4 $\Lambda_{\mu, \xi}^{k} / \lambda$

Using eqs. (80) and (7), we obtain $\Lambda_{\mu, \xi}^{k} / \lambda$ as

$$
\frac{\Lambda_{\mu, \xi}^{k}}{\lambda}=-2 \eta \frac{m}{M} \frac{1}{\hbar} \sqrt{\frac{M \hbar \omega_{\xi}}{2}} \sum_{j(\neq 1)}\left(x y\left|L_{\mu}\right| \Psi_{j}\right)\left(\Psi_{j}|\xi| x y\right),
$$

for $\mu, \xi=x, y, z$. The matrix element $\left(x y|\boldsymbol{L}| \Psi_{j}\right)$ is expressed by

$$
\left(x y\left|L_{x}\right| y z\right)=C^{2}\left[c _ { d } \left\langlex y \left|L_{x} \overline{|x z\rangle}+c_{d}\left(\overline{\left\langle x^{2}-y^{2}\right|} L_{x}|y z\rangle+\overline{\left\langle 3 z^{2}-r^{2}\right|} L_{x}|y z\rangle\right)+c_{p}^{2}\left(\overline{\langle y|} L_{x} \overline{|z\rangle}+\overline{\langle z|} L_{x}|y\rangle\right)\right.\right.\right.
$$


J. Phys. Soc. Jpn.

Full PaPer

$$
\begin{aligned}
& \left.+c_{d}^{2}\left(\overline{\langle x z|} L_{x} \overline{|x y\rangle}+\overline{\langle y z|} L_{x} \overline{\left|x^{2}-y^{2}\right\rangle}+\overline{\langle y z|} L_{x} \overline{\left|3 z^{2}-r^{2}\right\rangle}\right)\right]=\sqrt{3} c_{d}\left(1-c_{d}\right) C^{2} \mathrm{i} \\
& \left(x y\left|L_{x}\right| x z\right)=C^{2}\left[\left\langle x y\left|L_{x}\right| x z\right\rangle+c_{p}^{2}\left(\overline{\langle y|} L_{x} \overline{|z\rangle}+\overline{\langle z|} L_{x} \overline{|y\rangle}\right)+c_{d}^{2}\left(\overline{\langle x z|} L_{x} \overline{|x y\rangle}+\overline{\left\langle x^{2}-y^{2}\right|} L_{x} \overline{|y z\rangle}\right.\right. \\
& \left.\left.+\overline{\langle y z|} L_{x} \overline{\left|x^{2}-y^{2}\right\rangle}+\overline{\left\langle 3 z^{2}-r^{2}\right|} L_{x} \overline{|y z\rangle}+\overline{\langle y z|} L_{x} \overline{\left|3 z^{2}-r^{2}\right\rangle}\right)\right]=\left(c_{d}^{2}-1\right) C^{2} \mathrm{i}, \\
& \left(x y\left|L_{x}\right| x^{2}-y^{2}\right)=C^{2}\left[c_{d}\left(\left\langle x y\left|L_{x} \overline{|x z\rangle}+\overline{\langle y z|} L_{x}\right| x^{2}-y^{2}\right\rangle\right)+c_{p}^{2}\left(\overline{\langle y|} L_{x} \overline{|z\rangle}+\overline{\langle z|} L_{x} \overline{|y\rangle}\right)\right. \\
& \left.+c_{d}^{2}\left(\overline{\langle x z|} L_{x} \overline{|x y\rangle}+\overline{\left\langle x^{2}-y^{2}\right|} L_{x} \overline{|y z\rangle}+\overline{\left\langle 3 z^{2}-r^{2}\right|} L_{x} \overline{|y z\rangle}+\overline{\langle y z|} L_{x} \overline{\left|3 z^{2}-r^{2}\right\rangle}\right)\right] \\
& =2 c_{d}\left(c_{d}-1\right) C^{2} \mathrm{i}, \\
& \left(x y\left|L_{x}\right| 3 z^{2}-r^{2}\right)=C^{2}\left[c_{d}\left(\left\langle x y\left|L_{x} \overline{|x z\rangle}+\overline{\langle y z|} L_{x}\right| 3 z^{2}-r^{2}\right\rangle\right)+c_{p}^{2}\left(\overline{\langle y|} L_{x} \overline{|z\rangle}+\overline{\langle z|} L_{x} \overline{|y\rangle}\right)\right. \\
& \left.+c_{d}^{2}\left(\overline{\langle x z|} L_{x} \overline{|x y\rangle}+\overline{\left\langle x^{2}-y^{2}\right|} L_{x} \overline{|y z\rangle}+\overline{\langle y z|} L_{x} \overline{\left|x^{2}-y^{2}\right\rangle}+\overline{\left\langle 3 z^{2}-r^{2}\right|} L_{x} \overline{|y z\rangle}\right)\right] \\
& =(1+\sqrt{3}) c_{d}\left(c_{d}-1\right) C^{2} \mathrm{i}, \\
& \left.\left.+\overline{\langle x z|} L_{y} \overline{\left|x^{2}-y^{2}\right\rangle}+\overline{\left\langle 3 z^{2}-r^{2}\right|} L_{y} \overline{|x z\rangle}+\overline{\langle x z|} L_{y} \overline{\left|3 z^{2}-r^{2}\right\rangle}\right)\right]=\left(1-c_{d}^{2}\right) C^{2} \mathrm{i}, \\
& \left.+c_{d}^{2}\left(\overline{\langle y z|} L_{y} \overline{|x y\rangle}+\overline{\langle x z|} L_{y} \overline{\left|x^{2}-y^{2}\right\rangle}+\overline{\langle x z|} L_{y} \overline{\left|3 z^{2}-r^{2}\right\rangle}\right)\right]=(2-\sqrt{3}) c_{d}\left(1-c_{d}\right) C^{2} \mathrm{i},(\mathrm{C} \cdot 35) \\
& \left.+c_{d}^{2}\left(\overline{\langle y z|} L_{y} \overline{|x y\rangle}+\overline{\left\langle x^{2}-y^{2}\right|} L_{y} \overline{|x z\rangle}+\overline{\left\langle 3 z^{2}-r^{2}\right|} L_{y} \overline{|x z\rangle}+\overline{\langle x z|} L_{y} \overline{\left|3 z^{2}-r^{2}\right\rangle}\right)\right]=0,
\end{aligned}
$$

$$
\begin{aligned}
\left(x y\left|L_{y}\right| 3 z^{2}-r^{2}\right)= & C^{2}\left[c_{d}\left(\left\langle x y\left|L_{y} \overline{|y z\rangle}+\overline{\langle x z|} L_{y}\right| 3 z^{2}-r^{2}\right\rangle\right)+c_{p}^{2}\left(\overline{\langle x|} L_{y} \overline{|z\rangle}+\overline{\langle z|} L_{y} \overline{|x\rangle}\right)\right. \\
& \left.+c_{d}^{2}\left(\overline{\langle y z|} L_{y} \overline{|x y\rangle}+\overline{\left\langle x^{2}-y^{2}\right|} L_{y} \overline{|x z\rangle}+\overline{\langle x z|} L_{y} \overline{\left|x^{2}-y^{2}\right\rangle}+\overline{\left\langle 3 z^{2}-r^{2}\right|} L_{y} \overline{|x z\rangle}\right)\right] \\
= & (1+\sqrt{3}) c_{d}\left(1-c_{d}\right) C^{2} \mathrm{i},
\end{aligned}
$$

$\left(x y\left|L_{z}\right| y z\right)=C^{2}\left[c_{d}\left(\left\langle x y\left|L_{z}\right| \overline{\left.x^{2}-y^{2}\right\rangle}+\overline{\langle x z|} L_{z} \mid y z\right\rangle\right)+c_{p}^{2}\left(\overline{\langle x|} L_{z} \mid \overline{|y\rangle}+\overline{\langle y|} L_{z} \overline{|x\rangle}\right)\right.$

$$
\left.+c_{d}^{2}\left(\overline{\left\langle x^{2}-y^{2}\right|} L_{z} \overline{|x y\rangle}+\overline{\langle y z|} L_{z} \overline{|x z\rangle}\right)\right]=c_{d}\left(1-c_{d}\right) C^{2} \mathrm{i}
$$

$$
\begin{aligned}
\left(x y\left|L_{z}\right| x z\right)= & C^{2}\left[c_{d}\left(\left\langle x y\left|L_{z}\right| \overline{\left.x^{2}-y^{2}\right\rangle}+\overline{\langle y z|} L_{z} \mid x z\right\rangle\right)+c_{p}^{2}\left(\overline{\langle x|} L_{z} \overline{|y\rangle}+\overline{\langle y|} L_{z} \overline{|x\rangle}\right)\right. \\
& \left.+c_{d}^{2}\left(\overline{\langle x z|} L_{z} \overline{|y z\rangle}+\overline{\left\langle x^{2}-y^{2}\right|} L_{z} \overline{|x y\rangle}\right)\right]=3 c_{d}\left(1-c_{d}\right) C^{2} \mathrm{i},
\end{aligned}
$$$$
\left(x y\left|L_{z}\right| x^{2}-y^{2}\right)=C^{2}\left[\left\langle x y\left|L_{z}\right| x^{2}-y^{2}\right\rangle+c_{p}^{2}\left(\overline{\langle x|} L_{z} \overline{|y\rangle}+\overline{\langle y|} L_{z} \overline{|x\rangle}\right)\right.
$$$$
\left.+c_{d}^{2}\left(\overline{\left\langle x^{2}-y^{2}\right|} L_{z} \overline{|x y\rangle}+\overline{\langle x z|} L_{z} \overline{|y z\rangle}+\overline{\langle y z|} L_{z} \overline{|x z\rangle}\right)\right]=2\left(1-c_{d}^{2}\right) C^{2} \mathrm{i},
$$ 
J. Phys. Soc. Jpn.

Full PaPer

$$
\left.+c_{d}^{2}\left(\overline{\left\langle x^{2}-y^{2}\right|} L_{z} \overline{|x y\rangle}+\overline{\langle x z|} L_{z} \overline{|y z\rangle}+\overline{\langle y z|} L_{z} \overline{|x z\rangle}\right)\right]=2 c_{d}\left(1-c_{d}\right) C^{2} \mathrm{i}
$$

where $\left(\Psi_{j}|\boldsymbol{L}| \Psi_{j}\right)=0$ and $\left(\Psi_{j^{\prime}}|\boldsymbol{L}| \Psi_{j}\right)=-\left(\Psi_{j}|\boldsymbol{L}| \Psi_{j^{\prime}}\right)$ for $j \neq j^{\prime}{ }^{42}$ The matrix element $\left(\Psi_{j}|\xi| x y\right)$ is obtained as

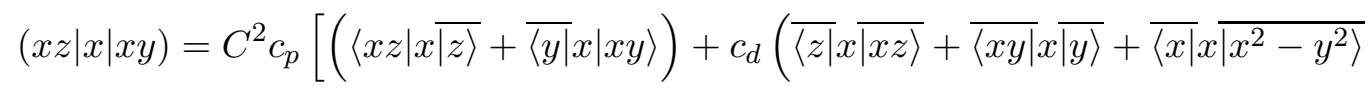

$$
\begin{aligned}
& \left.\left.+\overline{\left\langle x^{2}-y^{2}|x| x\right\rangle}+\overline{\langle x|} x \overline{\left|3 z^{2}-r^{2}\right\rangle}+\overline{\left\langle 3 z^{2}-r^{2}|x| x\right\rangle}\right)\right] \\
& (x z|y| x y)=C^{2} c_{p}\left[\overline{\langle x|} y|x y\rangle+c_{d}\left(\overline{\langle x y|} y \overline{|x\rangle}+\overline{\langle z|} y\left|\overline{|y z\rangle}+\overline{\langle y z|y| z\rangle}+\overline{\langle y|} y \overline{\left|x^{2}-y^{2}\right\rangle}+\overline{\left\langle x^{2}-y^{2}\right|} y\right| y\right\rangle\right. \\
& \left.\left.+\overline{\langle y|} y \overline{\left|3 z^{2}-r^{2}\right\rangle}+\overline{\left\langle 3 z^{2}-r^{2}\right|} y \overline{|y\rangle}\right)\right] \\
& (x z|z| x y)=C^{2} c_{p}\left[\langle x z| z \overline{|x\rangle}+c_{d}\left(\overline{\langle x|} z \overline{z \mid x z}+\overline{\langle y|} z \overline{|y z\rangle}+\overline{\langle y z|} z \overline{|y\rangle}+\overline{\langle z|} z \overline{\left|3 z^{2}-r^{2}\right\rangle}+\overline{\left\langle 3 z^{2}-r^{2}|z| z\right\rangle}\right)\right] \\
& (y z|x| x y)=C^{2} c_{p}\left[\overline{\langle y|} x|x y\rangle+c_{d}\left(\overline{\langle x y|} x \overline{|y\rangle}+\overline{\langle z|} x \overline{|x z\rangle}+\overline{\langle x z|x| z\rangle}+\overline{\langle x|} x \overline{\left|x^{2}-y^{2}\right\rangle}+\overline{\left\langle x^{2}-y^{2}|x| x\right\rangle}\right.\right. \\
& \left.\left.+\overline{\langle x|} x \overline{\left|3 z^{2}-r^{2}\right\rangle}+\overline{\left\langle 3 z^{2}-r^{2}|x| x\right\rangle}\right)\right], \\
& (y z|y| x y)=C^{2} c_{p}\left[(\langle y z|y| \overline{|z\rangle}+\overline{\langle x|} y \mid x y\rangle)+c_{d}\left(\overline{\langle z|} y|\overline{y z\rangle}+\overline{\langle x y|y| x\rangle}+\overline{\langle y|} y| \overline{\left.x^{2}-y^{2}\right\rangle}+\overline{\left\langle x^{2}-y^{2}|y| y\right\rangle}\right.\right. \\
& \left.\left.+\overline{\langle y|} y \overline{\left|3 z^{2}-r^{2}\right\rangle}+\overline{\left\langle 3 z^{2}-r^{2}\right|} y|y\rangle\right)\right] \\
& (y z|z| x y)=C^{2} c_{p}\left[\left\langle y z|z| \overline{|y\rangle}+c_{d}\left(\overline{\langle y|} z \overline{|y z\rangle}+\overline{\langle x z|z| x\rangle}+\overline{\langle x|} z \overline{x z\rangle}+\overline{\langle z|} z \overline{\left.3 z^{2}-r^{2}\right\rangle}+\overline{\left\langle 3 z^{2}-r^{2}|z| z\right\rangle}\right)\right],\right. \\
& \left(x^{2}-y^{2}|x| x y\right)=C^{2} c_{p}\left[\left(\left\langle x^{2}-y^{2}|x \overline{|x\rangle}+\overline{\langle y|} x| x y\right\rangle\right)+c_{d}\left(\overline{\langle x|} x \overline{\left|x^{2}-y^{2}\right\rangle}+\overline{\langle x y|x| y\rangle}+\overline{\langle x z|} x \overline{z\rangle}+\overline{\langle z|} x \overline{|x z\rangle}\right.\right. \\
& \left.\left.+\overline{\langle x|} x \overline{\left|3 z^{2}-r^{2}\right\rangle}+\overline{\left\langle 3 z^{2}-r^{2}|x| x\right\rangle}\right)\right], \\
& \left(x^{2}-y^{2}|y| x y\right)=C^{2} c_{p}\left[\left(\left\langle x^{2}-y^{2}|y| y\right\rangle+\overline{\langle x|} y|x y\rangle\right)+c_{d}\left(\overline{\langle y|}\left|\overline{y\left|x^{2}-y^{2}\right\rangle}+\overline{\langle x y|} y \overline{|x\rangle}+\overline{\langle z|} y\right| \overline{y z\rangle}+\overline{\langle y z|} y \mid \overline{|z\rangle}\right.\right. \\
& \left.\left.+\overline{\langle y|} y \overline{\left|3 z^{2}-r^{2}\right\rangle}+\overline{\left\langle 3 z^{2}-r^{2}|y| y\right\rangle}\right)\right] \\
& \left(x^{2}-y^{2}|z| x y\right)=C^{2} c_{d} c_{p}\left(\overline{\langle x|} z \overline{|x z\rangle}+\overline{\langle x z|} z \overline{|x\rangle}+\overline{\langle y|} z \overline{|y z\rangle}+\overline{\langle y z|} z|\overline{|y\rangle}+\overline{\langle z|} z| \overline{\left.3 z^{2}-r^{2}\right\rangle}+\overline{\left\langle 3 z^{2}-r^{2}|z| z\right\rangle}\right) \\
& \left(3 z^{2}-r^{2}|x| x y\right)=C^{2} c_{p}\left[\left(\left\langle 3 z^{2}-r^{2}|x \overline{|x\rangle}+\overline{\langle y|} x| x y\right\rangle\right)+c_{d}\left(\overline{\langle x|} x \overline{\left|3 z^{2}-r^{2}\right\rangle}+\overline{\langle x y|} x \overline{|y\rangle}+\overline{\langle x z|} x \overline{|z\rangle}\right.\right. \\
& \left.\left.+\overline{\langle z|} x \overline{|x z\rangle}+\overline{\left\langle x^{2}-y^{2}|x| x\right\rangle}+\overline{\langle x|} x \overline{\left|x^{2}-y^{2}\right\rangle}\right)\right]
\end{aligned}
$$

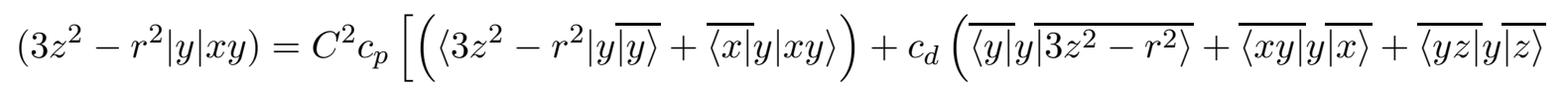

$$
\begin{aligned}
& \left.\left.+\overline{\langle z|} y \overline{|y z\rangle}+\overline{\left\langle x^{2}-y^{2}\right|} y \overline{|y\rangle}+\overline{\langle y|} y \overline{\left|x^{2}-y^{2}\right\rangle}\right)\right] \\
& \left(3 z^{2}-r^{2}|z| x y\right)=C^{2} c_{p}\left[\left\langle 3 z^{2}-r^{2}|z| \overline{|z\rangle}+c_{d}\left(\overline{\langle z|} z \overline{\left|3 z^{2}-r^{2}\right\rangle}+\overline{\langle x z|} z \mid \overline{|x\rangle}+\overline{\langle x|} z \overline{|x z\rangle}+\overline{\langle y z|} z \overline{|y\rangle}+\overline{\langle y|z| y z\rangle}\right)\right]\right.
\end{aligned}
$$


where $\left(\Psi_{j^{\prime}}|\xi| \Psi_{j}\right)=\left(\Psi_{j}|\xi| \Psi_{j^{\prime}}\right)$. Here, $\left\langle p_{j^{\prime}}|\xi| d_{j}\right\rangle$ is given by eqs. (C.24) - (C.28).

\section{$C .5 \quad \Lambda_{I, J}^{\mathrm{SF}} / D$}

Using eqs. (106) and (103), we obtain $\Lambda_{I, J}^{\mathrm{SF}} / D$ as

$$
\frac{\Lambda_{I, J}^{\mathrm{SF}}}{D}=\frac{\mathcal{L}_{I, J}^{\prime}}{\mathcal{L}_{Z, Z}^{(0)}-\left(\mathcal{L}_{X, X}^{(0)}+\mathcal{L}_{Y, Y}^{(0)}\right) / 2},
$$

with $I, J=X, Y, Z$. Here, $\mathcal{L}_{Z, Z}^{(0)}-\left(\mathcal{L}_{X, X}^{(0)}+\mathcal{L}_{Y, Y}^{(0)}\right) / 2$ (see eq. (C.17)) becomes

$$
\mathcal{L}_{Z, Z}^{(0)}-\frac{\mathcal{L}_{X, X}^{(0)}+\mathcal{L}_{Y, Y}^{(0)}}{2}=\left(\frac{1}{\Delta_{s}}-\frac{4}{\Delta_{m}}\right) C^{4} .
$$

Using eqs. (C.30) - (C.41), we obtain $\mathcal{L}_{I, J}^{\prime}$ of eqs. (97) (or $\mathcal{L}_{\mu, \nu}^{\prime}$ of eq. (91)) as

$$
\begin{aligned}
& \mathcal{L}_{X, Z}^{\prime}=\mathcal{L}_{x, z}^{\prime}=\frac{\left(x y\left|L_{x}\right| y z\right)\left(y z\left|L_{z}\right| x y\right)}{E_{x y}-E_{y z}}+\frac{\left(x y\left|L_{x}\right| x z\right)\left(x z\left|L_{z}\right| x y\right)}{E_{x y}-E_{x z}}+\frac{\left(x y\left|L_{x}\right| x^{2}-y^{2}\right)\left(x^{2}-y^{2}\left|L_{z}\right| x y\right)}{E_{x y}-E_{x^{2}-y^{2}}} \\
& +\frac{\left(x y\left|L_{x}\right| 3 z^{2}-r^{2}\right)\left(3 z^{2}-r^{2}\left|L_{z}\right| x y\right)}{E_{x y}-E_{3 z^{2}-r^{2}}}-\mathcal{L}_{x, z}^{(0)} \\
& =C^{4} c_{d}\left(c_{d}-1\right)\left[\frac{(3-\sqrt{3}) c_{d}^{2}+\sqrt{3} c_{d}-3}{\Delta_{s}}+\frac{4\left(c_{d}^{2}-1\right)}{\Delta_{m}}+\frac{2(1+\sqrt{3}) c_{d}\left(c_{d}-1\right)}{\Delta_{l}}\right], \\
& \mathcal{L}_{Y, Z}^{\prime}=\mathcal{L}_{y, z}^{\prime}=\frac{\left(x y\left|L_{y}\right| y z\right)\left(y z\left|L_{z}\right| x y\right)}{E_{x y}-E_{y z}}+\frac{\left(x y\left|L_{y}\right| x z\right)\left(x z\left|L_{z}\right| x y\right)}{E_{x y}-E_{x z}}+\frac{\left(x y\left|L_{y}\right| 3 z^{2}-r^{2}\right)\left(3 z^{2}-r^{2}\left|L_{z}\right| x y\right)}{E_{x y}-E_{3 z^{2}-r^{2}}}-\mathcal{L}_{y, z}^{(0)} \\
& =C^{4} c_{d}\left(c_{d}-1\right)\left[\frac{(-7+3 \sqrt{3}) c_{d}^{2}+(6-3 \sqrt{3}) c_{d}+1}{\Delta_{s}}+\frac{-(2+2 \sqrt{3}) c_{d}^{2}+(2+2 \sqrt{3}) c_{d}}{\Delta_{l}}\right], \\
& \mathcal{L}_{X, Y}^{\prime}=\mathcal{L}_{x, y}^{\prime}=\frac{\left(x y\left|L_{x}\right| y z\right)\left(y z\left|L_{y}\right| x y\right)}{E_{x y}-E_{y z}}+\frac{\left(x y\left|L_{x}\right| x z\right)\left(x z\left|L_{y}\right| x y\right)}{E_{x y}-E_{x z}}+\frac{\left(x y\left|L_{x}\right| 3 z^{2}-r^{2}\right)\left(3 z^{2}-r^{2}\left|L_{y}\right| x y\right)}{E_{x y}-E_{3 z^{2}-r^{2}}}-\mathcal{L}_{x, y}^{(0)} \\
& =C^{4} c_{d}\left(c_{d}-1\right)\left[\frac{(2-2 \sqrt{3}) c_{d}^{2}+2 \sqrt{3}-2}{\Delta_{s}}+\frac{(1+\sqrt{3})^{2}\left(c_{d}^{2}-c_{d}\right)}{\Delta_{l}}\right], \\
& \mathcal{L}_{X, X}^{\prime}=\mathcal{L}_{x, x}^{\prime}=\frac{\left(x y\left|L_{x}\right| y z\right)\left(y z\left|L_{x}\right| x y\right)}{E_{x y}-E_{y z}}+\frac{\left(x y\left|L_{x}\right| x z\right)\left(x z\left|L_{x}\right| x y\right)}{E_{x y}-E_{x z}}+\frac{\left(x y\left|L_{x}\right| x^{2}-y^{2}\right)\left(x^{2}-y^{2}\left|L_{x}\right| x y\right)}{E_{x y}-E_{x^{2}-y^{2}}} \\
& +\frac{\left(x y\left|L_{x}\right| 3 z^{2}-r^{2}\right)\left(3 z^{2}-r^{2}\left|L_{x}\right| x y\right)}{E_{x y}-E_{3 z^{2}-r^{2}}}-\mathcal{L}_{x, x}^{(0)} \\
& =C^{4} c_{d}\left\{-\frac{4 c_{d}+2}{\Delta_{s}}-\frac{4 c_{d}}{\Delta_{m}}-\frac{(1+\sqrt{3})^{2} c_{d}}{\Delta_{l}}+\left(c_{d}-2\right)\left[-\frac{4 c_{d}^{2}+2 c_{d}+1}{\Delta_{s}}-\frac{4 c_{d}^{2}}{\Delta_{m}}-\frac{(1+\sqrt{3})^{2} c_{d}^{2}}{\Delta_{l}}\right]\right\},
\end{aligned}
$$

$$
\begin{aligned}
\mathcal{L}_{Y, Y}^{\prime}=\mathcal{L}_{y, y}^{\prime} & =\frac{\left(x y\left|L_{y}\right| y z\right)\left(y z\left|L_{y}\right| x y\right)}{E_{x y}-E_{y z}}+\frac{\left(x y\left|L_{y}\right| x z\right)\left(x z\left|L_{y}\right| x y\right)}{E_{x y}-E_{x z}}+\frac{\left(x y\left|L_{y}\right| 3 z^{2}-r^{2}\right)\left(3 z^{2}-r^{2}\left|L_{y}\right| x y\right)}{E_{x y}-E_{3 z^{2}-r^{2}}}-\mathcal{L}_{y, y}^{(0)} \\
& =C^{4} c_{d}\left\{-\frac{(8-4 \sqrt{3}) c_{d}+2}{\Delta_{s}}-\frac{(1+\sqrt{3})^{2} c_{d}}{\Delta_{l}}\right.
\end{aligned}
$$


J. Phys. Soc. Jpn.

Full PaPer

$$
\begin{aligned}
+ & \left.\left(c_{d}-2\right)\left[-\frac{(8-4 \sqrt{3}) c_{d}^{2}+2 c_{d}+1}{\Delta_{s}}-\frac{(1+\sqrt{3})^{2} c_{d}^{2}}{\Delta_{l}}\right]\right\}, \\
\mathcal{L}_{Z, Z}^{\prime}=\mathcal{L}_{z, z}^{\prime}= & \frac{\left(x y\left|L_{z}\right| y z\right)\left(y z\left|L_{z}\right| x y\right)}{E_{x y}-E_{y z}}+\frac{\left(x y\left|L_{z}\right| x z\right)\left(x z\left|L_{z}\right| x y\right)}{E_{x y}-E_{x z}}+\frac{\left(x y\left|L_{z}\right| x^{2}-y^{2}\right)\left(x^{2}-y^{2}\left|L_{z}\right| x y\right)}{E_{x y}-E_{x^{2}-y^{2}}} \\
& +\frac{\left(x y\left|L_{z}\right| 3 z^{2}-r^{2}\right)\left(3 z^{2}-r^{2}\left|L_{z}\right| x y\right)}{E_{x y}-E_{3 z^{2}-r^{2}}}-\mathcal{L}_{z, z}^{(0)} \\
= & C^{4} c_{d}\left\{-\frac{10 c_{d}}{\Delta_{s}}-\frac{4\left(c_{d}+2\right)}{\Delta_{m}}-\frac{4 c_{d}}{\Delta_{l}}+\left(c_{d}-2\right)\left[-\frac{10 c_{d}^{2}}{\Delta_{s}}-\frac{4\left(c_{d}+1\right)^{2}}{\Delta_{m}}-\frac{4 c_{d}^{2}}{\Delta_{l}}\right]\right\},
\end{aligned}
$$

with $\quad \mathcal{L}_{x, z}^{(0)}=\mathcal{L}_{y, z}^{(0)}=\mathcal{L}_{x, y}^{(0)}=0 . \quad$ In addition, we have $\quad \mathcal{L}_{Z, X}^{\prime}=\mathcal{L}_{z, x}^{\prime}=\mathcal{L}_{x, z}^{\prime}, \quad \mathcal{L}_{Z, Y}^{\prime}=\mathcal{L}_{z, y}^{\prime}=\mathcal{L}_{y, z}^{\prime}$, $\mathcal{L}_{Y, X}^{\prime}=\mathcal{L}_{y, x}^{\prime}=\mathcal{L}_{x, y}^{\prime}{ }^{43}$ Note that $\mathcal{L}_{I, J}^{\prime}$ becomes 0 at $c_{d}=0$.

\section{$C .6 \quad G_{\xi}$ and $F / D$}

The coefficient $G_{\xi}$ is given by eq. (110):

$$
G_{\xi}=2 \eta \frac{\left\langle 3 \xi r^{-5}\right\rangle}{\left\langle r^{-3}\right\rangle} \sqrt{\frac{\hbar}{2 M \omega_{\xi}}},
$$

with $\xi=x, y, z$. Here, $\left\langle r^{-3}\right\rangle$ and $\left\langle 3 \xi r^{-5}\right\rangle$ are regarded as the respective ground-state expectation values:

$$
\begin{aligned}
& \left\langle r^{-3}\right\rangle=\left(x y\left|r^{-3}\right| x y\right) \\
& =C^{2}\left[\left\langle x y\left|r^{-3}\right| x y\right\rangle+c_{p}^{2}\left(\overline{\langle x|} r^{-3} \overline{|x\rangle}+\overline{\langle y|} r^{-3} \overline{|y\rangle}+\overline{\langle z|} r^{-3} \overline{|z\rangle}\right)+c_{d}^{2}\left(\overline{\langle y z|} r^{-3} \overline{|y z\rangle}+\overline{\langle x z|} r^{-3} \overline{|x z\rangle}\right.\right. \\
& \left.\left.+\overline{\left\langle x^{2}-y^{2}\right|} r^{-3} \overline{\left|x^{2}-y^{2}\right\rangle}+\overline{\left\langle 3 z^{2}-r^{2}\right|} r^{-3} \overline{\left|3 z^{2}-r^{2}\right\rangle}\right)\right], \\
& \left\langle 3 x r^{-5}\right\rangle=\left(x y\left|3 x r^{-5}\right| x y\right) \\
& =C^{2} c_{p}\left[\left(\left\langle x y\left|3 x r^{-5} \overline{|y\rangle}+\overline{\langle y|} 3 x r^{-5}\right| x y\right\rangle\right)+c_{d}\left(\overline{\left\langle x^{2}-y^{2}\right|} 3 x r^{-5} \overline{|x\rangle}+\overline{\langle x|} 3 x r^{-5} \overline{\left|x^{2}-y^{2}\right\rangle}\right.\right.
\end{aligned}
$$

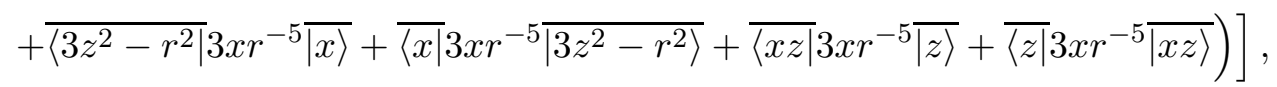

$$
\begin{aligned}
& \left\langle 3 y r^{-5}\right\rangle=\left(x y\left|3 y r^{-5}\right| x y\right) \\
& =C^{2} c_{p}\left[\left(\left\langle x y\left|3 y r^{-5} \overline{|x\rangle}+\overline{\langle x|} 3 y r^{-5}\right| x y\right\rangle\right)+c_{d}\left(\overline{\left\langle x^{2}-y^{2}\right|} 3 y r^{-5} \overline{|y\rangle}+\overline{\langle y|} 3 y r^{-5} \overline{\left|x^{2}-y^{2}\right\rangle}\right.\right.
\end{aligned}
$$

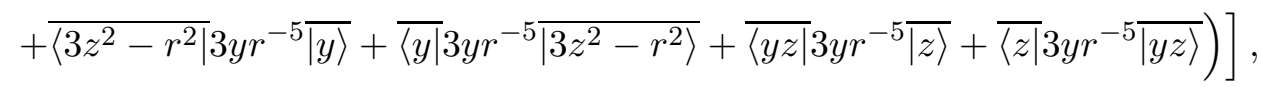

$$
\begin{aligned}
& \left\langle 3 z r^{-5}\right\rangle=\left(x y\left|3 z r^{-5}\right| x y\right) \\
& =C^{2} c_{d} c_{p}\left(\overline{\langle x z|} 3 z r^{-5} \overline{|x\rangle}+\overline{\langle x|} 3 z r^{-5} \overline{|x z\rangle}+\overline{\langle y z|} 3 z r^{-5} \overline{|y\rangle}+\overline{\langle y|} 3 z r^{-5} \overline{|y z\rangle}\right.
\end{aligned}
$$

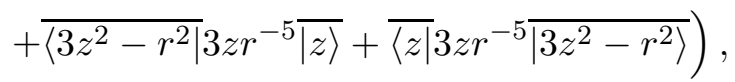

with

$$
\begin{aligned}
& \left\langle x\left|r^{-3}\right| x\right\rangle=2.42 \times 10^{30}, \\
& \left\langle x y\left|r^{-3}\right| x y\right\rangle=4.08 \times 10^{30}
\end{aligned}
$$


J. Phys. Soc. Jpn.

Full PaPer

$$
\begin{aligned}
& \left\langle 3 z^{2}-r^{2}\left|r^{-3}\right| 3 z^{2}-r^{2}\right\rangle=4.06 \times 10^{30} \\
& \left\langle x^{2}-y^{2}\left|r^{-3}\right| x^{2}-y^{2}\right\rangle=4.06 \times 10^{30} \\
& \left\langle x y\left|3 x r^{-5}\right| y\right\rangle=1.26 \times 10^{41} \\
& \left\langle x^{2}-y^{2}\left|3 x r^{-5}\right| x\right\rangle=1.26 \times 10^{41} \\
& \left\langle x^{2}-y^{2}\left|3 y r^{-5}\right| y\right\rangle=-1.26 \times 10^{41} \\
& \left\langle 3 z^{2}-r^{2}\left|3 x r^{-5}\right| x\right\rangle=-7.26 \times 10^{40} \\
& \left\langle 3 z^{2}-r^{2}\left|3 z r^{-5}\right| z\right\rangle=1.45 \times 10^{41}
\end{aligned}
$$

where eqs. (C.67) - (C·75) have been obtained by using eqs. (125) - (132).

\section{C.7 $\Lambda_{I, J ; \xi}^{\prime} / D$}

Using eqs. (111) and (103), we have

$$
\frac{\Lambda_{I, J ; \xi}^{\prime}}{D}=2 \eta \frac{\left\langle 3 \xi r^{-5}\right\rangle}{\left\langle r^{-3}\right\rangle} \sqrt{\frac{\hbar}{2 M \omega_{\xi}}} \frac{\mathcal{L}_{I, J}^{\prime}}{\mathcal{L}_{Z, Z}^{(0)}-\left(\mathcal{L}_{X, X}^{(0)}+\mathcal{L}_{Y, Y}^{(0)}\right) / 2},
$$

for $I, J=X, Y, Z$ and $\xi=x, y, z$. This $\Lambda_{I, J ; \xi}^{\prime} / D$ is also represented by $\Lambda_{I, J ; \xi}^{\prime} / D=G_{\xi} \Lambda_{I, J}^{\mathrm{SF}} / D$ (see eqs. (110) and (C.54)).

$C .8 \quad \Lambda_{\mu ; \nu, \xi}^{ \pm} / D$

Using eqs. (C·30) - (C.41), we express $\Lambda_{\mu ; \nu, \xi}^{ \pm} / D$ of eq. (81) with $\mu, \nu, \xi=x, y, z$ as

$$
\begin{aligned}
\frac{\Lambda_{x ; \nu, \xi}^{ \pm}}{D}= & {\left[\frac{\sqrt{3} c_{d}\left(1-c_{d}\right) \mathrm{i}(y z|\nu| x y)}{-\Delta_{s}} \kappa_{s, \xi}^{ \pm}+\frac{\left(c_{d}^{2}-1\right) \mathrm{i}(x z|\nu| x y)}{-\Delta_{s}} \kappa_{s, \xi}^{ \pm}+\frac{2 c_{d}\left(c_{d}-1\right) \mathrm{i}\left(x^{2}-y^{2}|\nu| x y\right)}{-\Delta_{m}} \kappa_{m, \xi}^{ \pm}\right.} \\
& \left.+\frac{(1+\sqrt{3}) c_{d}\left(c_{d}-1\right) \mathrm{i}\left(3 z^{2}-r^{2}|\nu| x y\right)}{-\Delta_{l}} \kappa_{l, \xi}^{ \pm}\right] /\left(\frac{1}{\Delta_{s}}-\frac{4}{\Delta_{m}}\right) C^{2},
\end{aligned}
$$

$\frac{\Lambda_{y ; \nu, \xi}^{ \pm}}{D}=\left[\frac{\left(1-c_{d}^{2}\right) \mathrm{i}(y z|\nu| x y)}{-\Delta_{s}} \kappa_{s, \xi}^{ \pm}+\frac{(2-\sqrt{3}) c_{d}\left(1-c_{d}\right) \mathrm{i}(x z|\nu| x y)}{-\Delta_{s}} \kappa_{s, \xi}^{ \pm}\right.$

$$
\left.+\frac{(1+\sqrt{3}) c_{d}\left(1-c_{d}\right) \mathrm{i}\left(3 z^{2}-r^{2}|\nu| x y\right)}{-\Delta_{l}} \kappa_{l, \xi}^{ \pm}\right] /\left(\frac{1}{\Delta_{s}}-\frac{4}{\Delta_{m}}\right) C^{2},
$$

$$
\begin{aligned}
\frac{\Lambda_{z ; \nu, \xi}^{ \pm}}{D}= & {\left[\frac{c_{d}\left(1-c_{d}\right) \mathrm{i}(y z|\nu| x y)}{-\Delta_{s}} \kappa_{s, \xi}^{ \pm}+\frac{3 c_{d}\left(1-c_{d}\right) \mathrm{i}(x z|\nu| x y)}{-\Delta_{s}} \kappa_{s, \xi}^{ \pm}+\frac{2\left(1-c_{d}^{2}\right) \mathrm{i}\left(x^{2}-y^{2}|\nu| x y\right)}{-\Delta_{m}} \kappa_{m, \xi}^{ \pm}\right.} \\
& \left.+\frac{2 c_{d}\left(1-c_{d}\right) \mathrm{i}\left(3 z^{2}-r^{2}|\nu| x y\right)}{-\Delta_{l}} \kappa_{l, \xi}^{ \pm}\right] /\left(\frac{1}{\Delta_{s}}-\frac{4}{\Delta_{m}}\right) C^{2},
\end{aligned}
$$

with

$$
\kappa_{u, \xi}^{ \pm}=\eta \frac{\mathrm{i}}{\hbar}\left(\frac{m}{M} \sqrt{\frac{M \hbar \omega_{\xi}}{2}} \pm \sqrt{\frac{\hbar}{2 M \omega_{\xi}}} \frac{m}{\hbar} \Delta_{u}\right)
$$


J. Phys. Soc. Jpn.

Full PAPeR

for $u=s, m$, and $l$. The matrix element $\left(\Psi_{j^{\prime}}|\nu| \Psi_{j}\right)$ is given by eqs. (C.42) - (C.53). We also note that $\Lambda_{\mu ; \nu, \xi}^{ \pm}$satisfies $\Lambda_{\mu ; \nu, \xi}^{ \pm}=-\Lambda_{\nu, \xi ; \mu}^{\mp}=\left(\Lambda_{\mu ; \nu, \xi}^{ \pm}\right)^{*}$ for $\mu, \nu, \xi=x, y, z,{ }^{44}$ where $\Lambda_{\nu, \xi ; \mu}^{\mp}$ is eq. (84). 
Table I. We show the presence or absence of the spin-atomic vibration interactions $V_{\mathrm{so} 2}^{(1)}, V_{\mathrm{so} 0, \mathrm{k}}^{(2)}$, $v_{\mathrm{so} 0, \mathrm{so} 1,1}^{(2)}, v_{\mathrm{so} 0, \mathrm{so} 1,2}^{(2)}$, and $V_{\mathrm{So} 0, \mathrm{so} 2}^{(2)}$, and the spin-flip Hamiltonian $v_{\mathrm{so} 0, \mathrm{so} 0,2}^{(2)}$ for each set of $\eta, c_{p}$, $c_{d}$. Here, $e_{1}-e_{j}=E_{1}-E_{j}$ is set by taking into account $\left|\Delta e_{1}-\Delta e_{j}\right| \ll\left|e_{1}-e_{j}\right|$ for $j \neq 1$. The spin-atomic vibration interaction (spin-flip Hamiltonian) is named SA (SF). The presence and absence are represented by $\sqrt{ }$ and $\times$, respectively. The operators and coefficients are also written for reference. The coefficient $\Lambda_{\mu ; \nu, \xi}^{ \pm}$contains both terms with $1 / \sqrt{f_{\xi}}$ and those with $\sqrt{f_{\xi}}$. The coefficient $\Lambda_{I, J}^{\mathrm{SF}}$ does not contain $\eta, f_{\xi}$, and $M$.

\begin{tabular}{|c|c|c|c|c|c|c|}
\hline Name & $\mathrm{SA}$ & $\mathrm{SA}$ & $\mathrm{SA}$ & $\mathrm{SA}$ & SA & $\mathrm{SF}$ \\
\hline \multirow[t]{2}{*}{ Energy } & $V_{\mathrm{so} 2}^{(1)}$ & $V_{\mathrm{so} 0, \mathrm{k}}^{(2)}$ & $v_{\mathrm{so} 0, \mathrm{so} 1,1}^{(2)}$ & $v_{\mathrm{so} 0, \mathrm{so} 1,2}^{(2)}$ & $V_{\mathrm{so} 0, \mathrm{so} 2}^{(2)}$ & $v_{\mathrm{So} 0, \mathrm{so} 0,2}^{(2)}$ \\
\hline & eq. (116) & eq. (114) & eq. (108) & eq. (109) & eq. (112) & eq. (102) \\
\hline Operator & $S_{I}\left(-a_{\xi}+a_{\xi}^{\dagger}\right)$ & $S_{I}\left(-a_{\xi}+a_{\xi}^{\dagger}\right)$ & $S_{I}^{2}\left(a_{\xi}+a_{\xi}^{\dagger}\right)$ & $S_{I} S_{J}\left(a_{\xi}+a_{\xi}^{\dagger}\right)$ & $\begin{array}{l}S_{I} S_{J} a_{\xi} \\
S_{I} S_{J} a_{\xi}^{\dagger}\end{array}$ & $S_{I} S_{J}$ \\
\hline \multirow[t]{5}{*}{ Coefficient } & $\Lambda_{\mu, \xi}^{(1)}$ & $\Lambda_{\mu, \xi}^{k}$ & $G_{\xi} D$ & $\Lambda_{I, J ; \xi}^{\prime}$ & $\Lambda_{\mu ; \nu, \xi}^{ \pm}$ & $\Lambda_{I, J}^{\mathrm{SF}}$ \\
\hline & & & $G_{\xi} E$ & & & \\
\hline & & & $G_{\xi} F$ & & & \\
\hline & $\propto \eta \lambda \sqrt{\frac{f_{\xi}}{M}}$ & $\propto \eta \lambda \sqrt{\frac{f_{\xi}}{M}}$ & $\propto \frac{\eta \lambda^{2}}{\sqrt{M f_{\xi}}}$ & $\propto \frac{\eta \lambda^{2}}{\sqrt{M f_{\xi}}}$ & $\propto \frac{\eta \lambda^{2}}{\sqrt{M}}$ & $\propto \lambda^{2}$ \\
\hline & eq. (55) & eq. (80) & eq. (110) & eq. (111) & eq. (81) & eq. (106) \\
\hline \multicolumn{7}{|l|}{$\eta=0$} \\
\hline$c_{d}=0, c_{p}=0$ & $\times$ & $\times$ & $\times$ & $\times$ & $\times$ & $\times$ \\
\hline$c_{d}=0, c_{p} \neq 0$ & $\times$ & $\times$ & $x$ & $\times$ & $\times$ & $\times$ \\
\hline$c_{d} \neq 0, c_{p}=0$ & $\times$ & $\times$ & $\times$ & $\times$ & $\times$ & $\sqrt{ }$ \\
\hline$c_{d} \neq 0, c_{p} \neq 0$ & $\times$ & $\times$ & $\times$ & $\times$ & $\times$ & $\sqrt{ }$ \\
\hline \multicolumn{7}{|l|}{$\eta \neq 0$} \\
\hline$c_{d}=0, c_{p}=0$ & $\times$ & $x$ & $\times$ & $\times$ & $\times$ & $\times$ \\
\hline$c_{d}=0, c_{p} \neq 0$ & $\sqrt{ }$ & $\sqrt{ }$ & $\sqrt{ }$ & $x$ & $\sqrt{ }$ & $x$ \\
\hline$c_{d} \neq 0, c_{p}=0$ & $\times$ & $\times$ & $x$ & $\times$ & $x$ & $\sqrt{ }$ \\
\hline$c_{d} \neq 0, c_{p} \neq 0$ & $\sqrt{ }$ & $\sqrt{ }$ & $\sqrt{ }$ & $\sqrt{ }$ & $\sqrt{ }$ & $\sqrt{ }$ \\
\hline
\end{tabular}


J. Phys. Soc. Jpn.

Full PAPER

\section{References}

1) J. Villain, F. Hartmann-Boutron, R. Sessoli, and A. Rettori: Europhys. Lett. 27 (1994) 159.

2) M. Misiorny and J. Barnas: Phys. Rev. B 77 (2008) 172414.

3) A. Ardavan, O. Rival, J. J. L. Morton, S. J. Blundell, A. M. Tyryshkin, G. A. Timco, and R. E. P. Winpenny: Phys. Rev. Lett. 98 (2007) 057201.

4) A. Caneschi, D. Gatteschi, R. Sessoli, A.-L. Barra, L.-C. Brunel, and M. Guillot: J. Am. Chem. Soc. 113 (1991) 5873.

5) R. Sessoli, H.-L. Tsai, A. R. Schake, S. Wang, J. B. Vincent, K. Folting, D. Gatteschi, G. Christou, and D. N. Hendrickson: J. Am. Chem. Soc. 115 (1993) 1804.

6) P. Politi, A. Rettori, F. Hartmann-Boutron, and J. Villain: Phys. Rev. Lett. 75 (1995) 537.

7) L. Thomas, F. Lionti, R. Ballou, D. Gatteschi, R. Sessoli, and B. Barbara: Nature 383 (1996) 145.

8) K. Takeda and K. Awaga: Phys. Rev. B 56 (1997) 14560; K. Takeda, K. Awaga, and T. Inabe, Phys. Rev. B 57 (1998) R11062.

9) A.-L. Barra, P. Debrunner, D. Gatteschi, Ch. E. Schulz, and R. Sessoli: Europhys. Lett. 35 (1996) 133.

10) C. F. Hirjibehedin, C.-Y. Lin, A. F. Otte, M. Ternes, C. P. Lutz, B. A. Jones, and A. J. Heinrich: Science 317 (2007) 1199.

11) C. F. Hirjibehedin, C. P. Lutz, and A. J. Heinrich: Science 312 (2006) 1021.

12) J. Park, A. N. Pasupathy, J. I. Goldsmith, C. Chang, Y. Yaish, J. R. Petta, M. Rinkoski, J. P. Sethna, H. D. Abruña, P. L. McEuen, and D. C. Ralph: Nature 417 (2002) 722.

13) D. Rugar, R. Budakian, H. J. Mamin, and B. W. Chui: Nature 430 (2004) 329.

14) N. Tsukahara, K. Noto, M. Ohara, S. Shiraki, N. Takagi, Y. Takata, J. Miyawaki, M. Taguchi, A. Chainani, S. Shin, and M. Kawai: Phys. Rev. Lett. 102 (2009) 167203.

15) Y. Yayon, V. W. Brar, L. Senapati, S. C. Erwin, and M. F. Crommie: Phys. Rev. Lett. 99 (2007) 067202 .

16) C. Etz, J. Zabloudil, P. Weinberger, and E. Y. Vedmedenko: Phys. Rev. B 77 (2008) 184425.

17) D. Serrate, P. Ferriani, Y. Yoshida, S.-W. Hla, M. Menzel, K. von Bergmann, S. Heinze, A. Kubetzka, and R. Wiesendanger: Nat. Nanotechnol. 5 (2010) 350.

18) S. Kokado, K. Ueda, K. Harigaya, and A. Sakuma: Phys. Rev. B 76 (2007) 054451.

19) S. Kokado, K. Harigaya, and A. Sakuma: Phys. Status Solidi C 6 (2009) 2113.

20) For example, see S. Amasha, K. MacLean, I. P. Radu, D. M. Zumbu, M. A. Kastner, M. P. Hanson, and A. C. Gossard: Phys. Rev. Lett. 100 (2008) 046803.

21) A. Zhao, Q. Li, L. Chen, H. Xiang, W. Wang, S. Pan, B. Wang, X. Xiao, J. Yang, J. G. Hou, and Q. Zhu: Science 309 (2005) 1542.

22) R. Z. Huang, V. S. Stepanyuk, A. L. Klavsyuk, W. Hergert, P. Bruno, and J. Kirschner: Phys. Rev. B 73 (2006) 153404.

23) J. H. Van Vleck: Phys. Rev. 57 (1940) 426.

24) J. H. Van Vleck: J. Chem. Phys. 7 (1939) 72.

25) R. D. Mattuck and M. W. P. Strandberg: Phys. Rev. 119 (1960) 1204.

26) R. Orbach: Proc. Phys. Soc. 77 (1961) 821.

27) A. M. Stoneham: Proc. Phys. Soc. 85 (1965) 107.

28) E. B. Tucker: Proc. IEEE 53 (1965) 1547. 
29) A. M. Stoneham: Theory of Defects in Solids (Oxford University Press, Oxford, 1975) Chap. 14. In particular, see Table 14.1.

30) F. Hartmann-Boutron, P. Politi, and J. Villain: Int. J. Mod. Phys. B 10 (1996) 2577.

31) D. Gatteschi, R. Sessoli, and J. Villain: Molecular Nanomagnets (Oxford University Press, Oxford, 2006) Chap. 5.

32) In the spin-phonon interaction, ${ }^{25,30}$ each magnetic ion (or each magnetic molecule) in the crystal has a single spin, where the exchange interactions between the spins are neglected. The spinphonon interaction originally represents an interaction between the spin located at the origin and the phonon of a lattice system.

33) K. Liu and S. Gao: Phys. Rev. B 74 (2006) 195433.

34) On the basis of the Coulomb energy between the $3 \mathrm{~d}$ electrons, $V_{\text {Coulomb }}=\sum_{\ell>\ell^{\prime}} e^{2} /\left(4 \pi \epsilon_{0}\left|\boldsymbol{r}_{\ell}-\boldsymbol{r}_{\ell^{\prime}}\right|\right)$, we show that the electrons prefer parallel spin alignment in the case of the weak crystal field. We now consider a case in which two electrons occupy the $(\zeta, \sigma)$ and $\left(\zeta^{\prime}, \sigma^{\prime}\right)$ states, respectively, where $\zeta(\sigma)$ represents the $\zeta$ orbital $(\sigma$ spin). With the use of the multiorbital Hubbard model, the energy may be roughly written as $2 \varepsilon_{d_{\zeta}}+U_{\zeta, \zeta}$ for $\zeta=\zeta^{\prime}$ and $\sigma \neq \sigma^{\prime}$, and $\varepsilon_{d_{\zeta}}+\varepsilon_{d_{\zeta^{\prime}}}+U_{\zeta, \zeta^{\prime}}-J_{\zeta, \zeta^{\prime}} \delta_{\sigma, \sigma^{\prime}}$ for $\zeta \neq \zeta^{\prime}$. Here, $\varepsilon_{d_{\zeta}}\left(\varepsilon_{d_{\zeta^{\prime}}}\right)$ is an energy of the $\zeta$ orbital ( $\zeta^{\prime}$ orbital), where $\varepsilon_{d_{\zeta}}<\varepsilon_{d_{\zeta^{\prime}}}$ (see eq. (39)). In addition, $U_{\zeta, \zeta^{\prime}}$ is an intra-atomic Coulomb energy defined by $U_{\zeta, \zeta^{\prime}}=\iint d_{\zeta}^{*}(\ell) d_{\zeta^{\prime}}^{*}\left(\ell^{\prime}\right) e^{2} /\left(4 \pi \epsilon_{0}\left|\boldsymbol{r}_{\ell}-\boldsymbol{r}_{\ell^{\prime}}\right|\right) d_{\zeta}(\ell) d_{\zeta^{\prime}}\left(\ell^{\prime}\right) \mathrm{d} \boldsymbol{r}_{\ell} \mathrm{d} \boldsymbol{r}_{\ell^{\prime}}(>0)$, and $J_{\zeta, \zeta^{\prime}}$ is an intra-atomic exchange energy defined by $J_{\zeta, \zeta^{\prime}}=\iint d_{\zeta}^{*}(\ell) d_{\zeta^{\prime}}^{*}\left(\ell^{\prime}\right) e^{2} /\left(4 \pi \epsilon_{0}\left|\boldsymbol{r}_{\ell}-\boldsymbol{r}_{\ell^{\prime}}\right|\right) d_{\zeta}\left(\ell^{\prime}\right) d_{\zeta^{\prime}}(\ell) \mathrm{d} \boldsymbol{r}_{\ell} \mathrm{d} \boldsymbol{r}_{\ell^{\prime}}(>0)$, where $d_{\zeta}(\ell)$ represents the $3 \mathrm{~d}$ orbital (see eq. (36)). We assume the cases of $U_{\zeta, \zeta}=U_{\zeta, \zeta^{\prime}}$ and the weak crystal field with $\varepsilon_{d_{\zeta^{\prime}}}-\varepsilon_{d_{\zeta}}<J_{\zeta, \zeta^{\prime}}$, where $\varepsilon_{d_{\zeta^{\prime}}}-\varepsilon_{d_{\zeta}}$ is the crystal field splitting energy. We then obtain the relation of $\varepsilon_{d_{\zeta}}+\varepsilon_{d_{\zeta^{\prime}}}+U_{\zeta, \zeta^{\prime}}-J_{\zeta, \zeta^{\prime}}<2 \varepsilon_{d_{\zeta}}+U_{\zeta, \zeta}$, which reduces to $\varepsilon_{d_{\zeta^{\prime}}}-\varepsilon_{d_{\zeta}}<J_{\zeta, \zeta^{\prime}}$. The electrons thus occupy the orbitals, keeping their spins parallel. For the multiorbital Hubbard model, see L. M. Roth: Phys. Rev. 149 (1966) 306.

35) K. Yosida: Theory of Magnetism (Springer Series, New York, 1998) Chap. 1.

36) According to Slater's rule, ${ }^{37} Z_{\text {eff }}$ of Fe is evaluated to be 6.25 .

37) J. C. Slater: Phys. Rev. 36 (1930) 57.

38) For example, see Appendix . As found from eq. (B.6), the magnitude of vibration displacement decreases with increasing mass and force constant. When the vibration of the surrounding ions is considered, the magnetic ion (the surrounding ions) in this Appendix should be replaced with the surrounding ions (the other ions adjacent to them in the substrate).

39) M.-C. Desjonquères and D. Spanjaard: Concepts in Surface Physics (Springer-Verlag, Berlin, 1995) 2nd ed., p. 505.

40) See chap. 3 in ref. 35.

41) M. H. L. Pryce: Proc. Phys. Soc. A 63 (1950) 25.

42) We here use the wave functions of $3 d$ orbitals, i.e., $d_{1}=x y f_{3,2}(r), d_{2}=x z f_{3,2}(r), d_{3}=y z f_{3,2}(r)$, $d_{4}=\left[\left(x^{2}-y^{2}\right) / 2\right] f_{3,2}(r)$, and $d_{5}=\left[\left(3 z^{2}-r^{2}\right) / 2 \sqrt{3}\right] f_{3,2}(r)$ (see eqs. (125) - (129)), where $d_{\zeta}(\zeta=1-5)$ is a real function. We then have $\left\langle d_{\zeta}|\boldsymbol{L}| d_{\zeta}\right\rangle=0$ and $\left\langle d_{\zeta^{\prime}}|\boldsymbol{L}| d_{\zeta}\right\rangle=-\left\langle d_{\zeta}|\boldsymbol{L}| d_{\zeta^{\prime}}\right\rangle$ for $\zeta \neq \zeta^{\prime}$, because $\boldsymbol{L}$ is purely imaginary and a Hermitian operator. Next, we consider $\Psi_{j}=\sum_{\zeta} c_{\zeta}^{(j)} d_{\zeta}$, where $\Psi_{j}$ is chosen to be a real function. We have $\left(\Psi_{j}|\boldsymbol{L}| \Psi_{j}\right)=$ $\sum_{\zeta^{\prime}} \sum_{\zeta^{\prime}} c_{\zeta^{\prime}}^{(j)} c_{\zeta}^{(j)}\left\langle d_{\zeta^{\prime}}|\boldsymbol{L}| d_{\zeta}\right\rangle=\sum_{\zeta^{\prime}>\zeta} c_{\zeta^{\prime}}^{(j)} c_{\zeta}^{(j)}\left(\left\langle d_{\zeta^{\prime}}|\boldsymbol{L}| d_{\zeta}\right\rangle+\left\langle d_{\zeta}|\boldsymbol{L}| d_{\zeta^{\prime}}\right\rangle\right)=0$. Furthermore, when $j \neq j^{\prime}$, we obtain $\left(\Psi_{j^{\prime}}|\boldsymbol{L}| \Psi_{j}\right)=\sum_{\zeta^{\prime}} \sum_{\zeta} c_{\zeta^{\prime}}^{\left(j^{\prime}\right)} c_{\zeta}^{(j)}\left\langle d_{\zeta^{\prime}}|\boldsymbol{L}| d_{\zeta}\right\rangle=\sum_{\zeta} \sum_{\zeta^{\prime}} c_{\zeta}^{(j)} c_{\zeta^{\prime}}^{\left(j^{\prime}\right)}\left(-\left\langle d_{\zeta}|\boldsymbol{L}| d_{\zeta^{\prime}}\right\rangle\right)=-\left(\Psi_{j}|\boldsymbol{L}| \Psi_{j^{\prime}}\right)$. 
43) Regarding $\mathcal{L}_{\mu, \nu}$ of eq. (79), we obtain $\mathcal{L}_{\mu, \nu}=\mathcal{L}_{\nu, \mu}$ using $\left(\Psi_{j^{\prime}}\left|L_{\mu}\right| \Psi_{j}\right)=-\left(\Psi_{j}\left|L_{\mu}\right| \Psi_{j^{\prime}}\right){ }^{42}$ In a similar way, $\mathcal{L}_{\mu, \nu}^{(0)}=\mathcal{L}_{\nu, \mu}^{(0)}$ is obtained using $\left\langle\Psi_{j^{\prime}}\left|L_{\mu}\right| \Psi_{j}\right\rangle=-\left\langle\Psi_{j}\left|L_{\mu}\right| \Psi_{j^{\prime}}\right\rangle{ }^{42}$ From $\mathcal{L}_{\mu, \nu}^{\prime}=\mathcal{L}_{\mu, \nu}-\mathcal{L}_{\mu, \nu}^{(0)}$, we have $\mathcal{L}_{\mu, \nu}^{\prime}=\mathcal{L}_{\nu, \mu}^{\prime}$.

44) Using $\left(\Psi_{j^{\prime}}\left|L_{\mu}\right| \Psi_{j}\right)=-\left(\Psi_{j}\left|L_{\mu}\right| \Psi_{j^{\prime}}\right)$ (see ref. 42) and $\left(\Psi_{j^{\prime}}\left|F_{\nu, \xi}^{ \pm}\right| \Psi_{j}\right)=\left(\Psi_{j}\left|F_{\nu, \xi}^{\mp}\right| \Psi_{j^{\prime}}\right)$ of eq. (82), we obtain $\Lambda_{\mu ; \nu, \xi}^{ \pm}=\eta \lambda^{2}(1 / \hbar) \sum_{j(\neq 1)}\left(\Psi_{1}\left|L_{\mu}\right| \Psi_{j}\right)\left(\Psi_{j}\left|F_{\nu, \xi}^{ \pm}\right| \Psi_{1}\right) /\left(E_{1}-E_{j}\right)=-\eta \lambda^{2}(1 / \hbar)$ $\sum_{j(\neq 1)}\left(\Psi_{1}\left|F_{\nu, \xi}^{\mp}\right| \Psi_{j}\right) \quad\left(\Psi_{j}\left|L_{\mu}\right| \Psi_{1}\right) /\left(E_{1}-E_{j}\right)=-\Lambda_{\nu, \xi ; \mu}^{\mp}$. In addition, we have $\left(\Lambda_{\mu ; \nu, \xi}^{ \pm}\right)^{*}=$ $\eta \lambda^{2}(1 / \hbar) \sum_{j(\neq 1)}\left(\Psi_{1}\left|\left(F_{\nu, \xi}^{ \pm}\right)^{\dagger}\right| \Psi_{j}\right)\left(\Psi_{j}\left|\left(L_{\mu}\right)^{\dagger}\right| \Psi_{1}\right) /\left(E_{1}-E_{j}\right)=\eta \lambda^{2}(1 / \hbar) \sum_{j(\neq 1)}\left(\Psi_{1}\left|\left(-F_{\nu, \xi}^{\mp}\right)\right| \Psi_{j}\right)$ $\left(\Psi_{j}\left|L_{\mu}\right| \Psi_{1}\right) /\left(E_{1}-E_{j}\right)=-\Lambda_{\nu, \xi ; \mu}^{\mp}=\Lambda_{\mu ; \nu, \xi}^{ \pm}$

45) J. Kondo: Prog. Theor. Phys. 24 (1960) 161.

46) See chap. 2 in ref. 31.

47) In the case of $c_{d}=c_{p}=0.15$, the total sum of existing probabilities of $\overline{d_{m}}$ and $\overline{p_{n}}$ in $\psi_{j}$ of Eq. (122) is given by $C^{2}\left(\sum_{m=1(\neq j)}^{5}\left|c_{d}\right|^{2}+\sum_{n=1}^{3}\left|c_{p}\right|^{2}\right)=0.14$. Here, $c_{p}=0.15$, which leads to $C^{2} \sum_{n=1}^{3}\left|c_{p}\right|^{2}=0.058$, is roughly set by drawing upon the Fe $4 \mathrm{p}$ partial density of states (DOS) in the energy region of $\mathrm{Fe} 3 \mathrm{~d}$ band of $\mathrm{Fe}_{4} \mathrm{~N}$. As to the DOS of $\mathrm{Fe}_{4} \mathrm{~N}$, see A. Sakuma: J. Phys. Soc. Jpn. 60 (1991) 2007. As to a schematic illustration of the partial DOS's of $\mathrm{Fe}_{4} \mathrm{~N}$, see S. Kokado, N. Fujima, K. Harigaya, H. Shimizu, and A. Sakuma: Phys. Rev. B 73 (2006) 172410.

48) For example, see J. A. Stroscio and R. J. Celotta: Science 306 (2004) 242. In this literature, the typical vibration frequencies are considered to range from $0.01 \mathrm{THz}$ to $10 \mathrm{THz}$.

49) On the basis of the coefficient in eq. (7) in ref. $28, c_{q}$ has been written as eq. (139).

50) M. N. Leuenberger and D. Loss: Phys. Rev. B 61 (2000) 1286.

51) A. Abragam and B. Bleaney: Electron Paramagnetic Resonance of Transition Ions (Clarendon Press, Oxford, 1970) Sect. 10.4.

$52)$ C. P. Slichter: Principles of Magnetic Resonance (Springer, New York, 1990) 3rd ed., Chap. 5.

53) C. Kittel: Introduction to Solid State Physics (John Wiley \& Sons, New York, 1986) 6th ed., p. 372.

54) For example, see chap. 3 in ref. 53.

55) For example, see M. T. Dove: Structure and Dynamics: An Atomic View of Materials (Oxford University Press, Oxford, 2003) Sects. 9.3 and N.

56) For example, see J. M. Ziman: Electrons and Phonons (Oxford University Press, Oxford, 1960) Chap. I. 